\title{
Diffractive photoproduction of $J / \psi$ and $\Upsilon$ using holographic QCD: Gravitational form factors and GPD of gluons in the proton
}

\author{
Kiminad A. Mamo* and Ismail Zahed \\ Department of Physics and Astronomy, Stony Brook University, Stony Brook, New York 11794-3800, USA
}

(Received 6 December 2019; accepted 9 March 2020; published 3 April 2020)

\begin{abstract}
We present a holographic analysis of diffractive photoproducton of charmonium $J / \psi$ and upsilonium $\Upsilon$ on a proton, considered as a bulk Dirac fermion, for all ranges of $\sqrt{s}$, i.e., from near threshold to very high energy. Using the bulk wave functions of the proton and vector mesons, within holographic QCD, and employing Witten diagrams in the bulk, we compute the diffractive photoproduction amplitude of $J / \psi$ and $\Upsilon$. The holographic amplitude shows elements of the strictures of vector meson dominance. It is dominated by the exchange of a massive graviton or $2^{++}$glueball resonances near threshold, and its higher spin-j counterparts that reggeize at higher energies. Both the differential and total cross sections are controlled by the gravitational form factor $A(t)$, and compare well to the recent results reported by the GlueX Collaboration near threshold and the world data at large $\sqrt{s}$. The holographic gravitational form factors, including the D-term, which is due to the exchange of massive spin- 0 glueballs, are in good agreement with lattice simulations. We use it to extract the holographic pressure and shear forces inside the proton. Finally, using a pertinent integral representation of the holographic gravitational form factor $A(t)$ near threshold, and its Pomeron counterpart way above threshold, we extract the generalized parton distribution of gluons inside the proton at different resolutions.
\end{abstract}

DOI: 10.1103/PhysRevD.101.086003

\section{INTRODUCTION}

Exclusive production of heavy mesons such as charmonia and bottomonia through the use of photo- or electroproduction processes provides the optimal framework for diffractive physics. In the limit when the coherence length of the virtual photon is large in comparison to the proton size, the scattering virtual photon on a hadron is equivalent to the scattering of a hadron onto a hadron. The process is mostly dominated by the exchange of gluons with vacuum quantum numbers, leading to a slowly rising cross section at high energy. The rise is due to the exchange of a Pomeron, an effective object lying on the highest Regge trajectory. First principle perturbative QCD calculations $[1,2]$ provide insights to the nature of this exchange, although the softness of the exchange suggests an altogether nonperturbative approach.

Soft electroproduction on a nucleon is analogous to a hadron of varying size scattering off a nucleon, with a virtual photon wave function of squared transverse size

\footnotetext{
kiminad.mamo@stonybrook.edu

ismail.zahed@stonybrook.edu
}

Published by the American Physical Society under the terms of the Creative Commons Attribution 4.0 International license. Further distribution of this work must maintain attribution to the author(s) and the published article's title, journal citation, and DOI. Funded by SCOAP ${ }^{3}$.
$1 / Q^{2}$. In the photoproduction limit with $Q^{2} \rightarrow 0$, the size is hadronic and nonperturbative physics applies. The diffractive and nonperturbative production process whereby the soft virtual photon turns to a heavy meson is analogous to the scattering of two dipoles with light-cone wave functions for the in-out virtual photon states. It is inherently nonperturbative at small $Q^{2}$. Throughout, we will focus on electroproduction close to the photon point or photoproduction for heavy mesons such as charmonium and bottomium.

Holographic QCD provides a nonperturbative framework for discussing structure and scattering of hadrons. It stems from a conjecture that observables in strongly coupled gauge theories in the limit of a large number of colors can be determined from classical fields interacting through gravity in generally an anti-de-Sitter space in higher dimensions [3]. The original conjecture was put forth and demonstrated for conformal $\mathcal{N}=4$ Yang-Mills theory, and argued by many to hold under some assumptions for nonconformal gauge theories such as QCD. Exclusive production of heavy mesons has been analyzed in the context of holographic QCD at high energy [4,5], where the exchange reggeizes [6-13]. Diffractive production of vector mesons in the nonholographic context can be found in [14].

Recently, the GlueX Collaboration has put forth measurements of threshold charmonium production using virtual photons close to the photon point [15]. Additional measurements at JLab in this channel with higher accuracy 
using the SoLID detector should improve further the statistics [16]. One purpose of these experiments is the extraction of the gluonic component entering the composition of the nucleon mass. In this spirit, a new analysis of these threshold data was carried in [17,18] using a hybrid holographic construction combining general QCD arguments and lattice results. One of the purposes of this paper is to carry an analysis of the new GlueX data near threshold [15] and the existing world data well above threshold, all within a holographic QCD model using the bottom-up approach. This analysis complements the earlier investigations in $[4,5]$ at high energy, all the way to threshold. For completeness, we note the earlier suggestion to use the photoproduction process near threshold to probe the gluon content of the nucleon [19].

The holographic photoproduction amplitude is dominated by the exchange of a massive $2^{++}$graviton at threshold, and higher spin-j exchanges away from threshold that rapidly reggeize. The $0^{++}$glueballs are found to decouple owing to their vanishing coupling to the virtual photons, while the dilatons are shown to decouple from the bulk Dirac fermion. At threshold, the holographic photoproduction amplitude directly probes a pertinent gravitational form factor which maps on the gluonic contribution to the energy-momentum tensor of the nucleon as a Dirac fermion in the bulk.

This paper consists of several new results: (i) The derivation of all three holographic gravitational form factors and their comparison to recent lattice data. (ii) The derivation of the gluonic pressure and shear forces inside the proton. (iii) The derivation that the holographic processes $\gamma p \rightarrow V p$ and $\gamma p \rightarrow \gamma^{*} p$ are related in bulk by vector meson dominance (VMD). (iv) The derivation of the holographic photoproduction differential and total cross sections for $J / \Psi$ and their comparison to current data for all energies. (v) The derivation that the threshold cross section is dominated by only one invariant gravitational form factor $A(t)$, due to the exchange of a $2^{++}$glueball in bulk. (vi) The extraction of the value of $A(0)$ from the data for different brane embeddings. (vii) The derivation of the holographic gluonic generalized parton distribution (GPD) of the nucleon as a bulk Dirac fermion. (viii) The prediction for the diffractive photoproduction of $\Upsilon$.

The organization of the paper is as follows: In Sec. II we review the kinematics for a general $2 \rightarrow 2$ process. In Sec. III, we detail the general structures of the Witten diagrams for exclusive process, like the diffractive photoproduction of $J / \psi$, by using the bulk wave functions of hadrons in holographic QCD. In Sec. IV, we introduce in detail the bottom holographic model we use, and derive the bulk vertices for the Witten diagrams from the bulk action of the model. In Sec. V, we derive the holographic gravitational form factors using Witten diagrams, and campare them to the recent lattice results. In Sec. VI, we use our holographic D-term to calculate the pressure distribution and shear forces inside the proton. In Sec. VII, we show how vector meson dominance holds in the present holographic construction, and derive the scattering amplitude for the diffractive photoproduction by approximating the bulk-to-bulk glueball propagator near the boundary which will enable us to write down the scattering amplitude explicitly in terms of the gravitational form factor $A(t)$ of spin-2 glueball exchanges. In Sec. VIII, the photoproduction differential and total cross sections close to the photon point are detailed at threshold in the single graviton exchange limit. In Sec. IX, we generalize the result beyond threshold through reggeization by including the higher spin- $\mathrm{j}$ exchanges and their resummation. In Sec. X, we derive the gluonic GPD from a pertinent integral representation of the form factor $A(t)$. Our conclusions are in Sec. XI, and details of the calculations are given in several appendixes.

\section{KINEMATICS OF THE $\gamma^{*} p \rightarrow V p$ PROCESS}

Throughout, we will refer to real and virtual photoproduction by $\gamma^{*}$ in the general presentation, but we will specialize to photoproduction in most of the specific analyses and results. All our arguments extend readily to diffractive electroproduction of heavy mesons $V=J / \Psi, \Upsilon$ with minor changes.

We start by briefly reviewing the kinematics for the process $\gamma^{*} p \rightarrow V p$. We first define the Lorentz scalars as $s=W^{2}=\left(p_{1}+q_{1}\right)^{2}$, and $t=\left(p_{1}-p_{2}\right)^{2}=\left(q_{1}-q_{2}\right)^{2}$ where $q_{1,2}$ are the four-vectors of the virtual photon and vector meson, respectively (note that we occasionally use the notation $q \equiv q_{1}$ and $q^{\prime} \equiv q_{2}$ ), and $p_{1,2}$ are the four vector of the proton. Throughout we will work with mostly negative signature, i.e., $\eta_{\mu \nu}=(+1,-1,-1,-1)$. Note that our convention is different from the mostly positive signature used in most holographic analyses.

We will work in the center-of-mass frame of the pair composed of the virtual photon $\gamma^{*}$ and the proton. In this frame, one can derive the mathematical relationships between the three-momenta of the virtual photon and vector meson $\left(\mathbf{q}_{\gamma}, \mathbf{q}_{V}\right)$ and Lorentz scalars $\left(s, t, q_{1}^{2}=-Q^{2}\right.$, $q_{2}^{2}=M_{V}^{2}, p_{1}^{2}=p_{2}^{2}=m_{N}^{2}$ ) as (see, for example, Eqs. (11.2-4) in [20])

$$
\begin{array}{r}
\left|\mathbf{q}_{\gamma}\right|=\frac{1}{2 \sqrt{s}} \sqrt{s^{2}-2\left(-Q^{2}+m_{N}^{2}\right) s+\left(-Q^{2}-m_{N}^{2}\right)^{2}}, \\
\left|\mathbf{q}_{V}\right|=\frac{1}{2 \sqrt{s}} \sqrt{s^{2}-2\left(M_{V}^{2}+m_{N}^{2}\right) s+\left(M_{V}^{2}-m_{N}^{2}\right)^{2}},
\end{array}
$$

and

$$
t=-Q^{2}+M_{V}^{2}-2 E_{\gamma} E_{V}+2\left|\mathbf{q}_{\gamma}\right|\left|\mathbf{q}_{V}\right| \cos \theta
$$

Here $E_{\gamma}=\left(-Q^{2}+\mathbf{q}_{\gamma}^{2}\right)^{\frac{1}{2}}$ is the energy of the virtual photon, and $E_{V}=\left(M_{V}^{2}+\mathbf{q}_{V}^{2}\right)^{\frac{1}{2}}$ is the energy of the vector meson. 


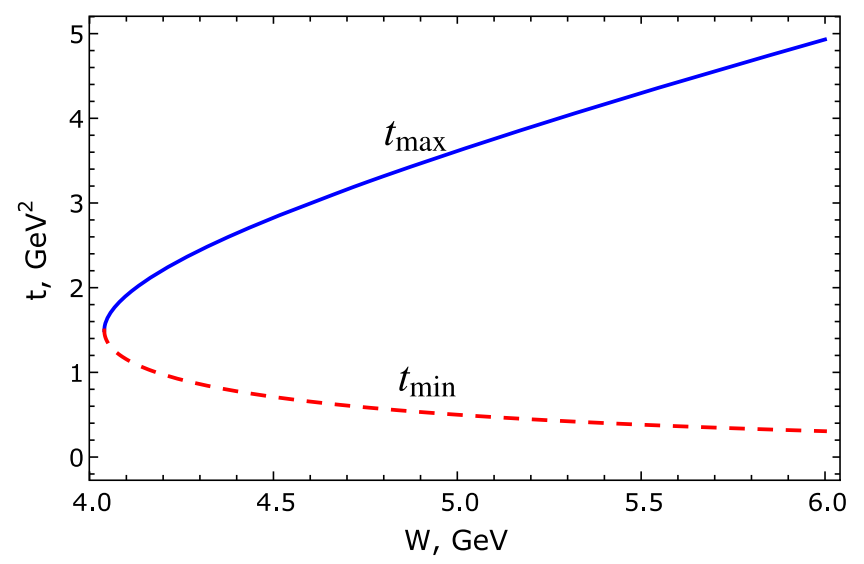

FIG. 1. $t_{\min }$ and $t_{\max }$ vs $W=\sqrt{s}$ for $M_{V}=M_{J / \psi}=3.10 \mathrm{GeV}$, $m_{N}=0.94 \mathrm{GeV}$, and $Q=0$. Note that at the threshold energy $W_{t r}=\sqrt{s_{t r}}=m_{N}+M_{V}=4.04 \mathrm{GeV}$, we have $t_{\min }=t_{\max }$.

The t-transfer at low $\sqrt{s}$ is bounded by $t_{\min } \equiv|t|_{\cos \theta=+1} \mid$ and $t_{\max } \equiv|t|_{\cos \theta=-1} \mid$ as illustrated in Fig. 1 .

We now note that at threshold and for example $V=J / \Psi$ with $s_{\text {tr }}=\left(m_{N}+M_{V}\right)^{2}=4.04 \mathrm{GeV}^{2}$

$$
\begin{aligned}
-t_{\min }\left(s=s_{\text {tr }}\right) & =\frac{m_{N} M_{V}^{2}}{m_{N}+M_{V}} \\
& =1.5^{2} \mathrm{GeV}^{2} \ll 4.04^{2} \mathrm{GeV}^{2}=s_{\text {tr }}
\end{aligned}
$$

and away from threshold

$$
-t_{\min }\left(s \gg s_{\text {tr }}\right) \sim\left(\frac{m_{N} M_{V}}{s}\right)^{2} \ll s .
$$

The photoproduction kinematics for charmonium and also bottomium is dominated by the diffractive process all the way to threshold.

The differential cross section for the photoproduction process $\gamma^{*} p \rightarrow V p$ is given by (see for example, Eq. (11.34) in [20])

$$
\frac{d \sigma}{d t}=\frac{e^{2}}{64 \pi s\left|\mathbf{q}_{\gamma}\right|^{2}}\left|\mathcal{A}_{\gamma * p \rightarrow V p}(s, t)\right|^{2}
$$

and the total cross section for small $\sqrt{s}$ close to threshold is

$$
\sigma(s)=\int_{t_{\min }}^{t_{\max }} d t\left(\frac{d \sigma}{d t}\right) .
$$

We now show how to use Witten diagrams in AdS with bulk wave functions for the vector mesons, bulk-toboundary and bulk-to-bulk propagators within pertinent holographic models in the bottom-up approach.

\section{HOLOGRAPHIC PHOTOPRODUCTION OF VECTOR MESONS}

The diffractive amplitude for the photoproduction of a vector meson, in a given holographic model of QCD, can

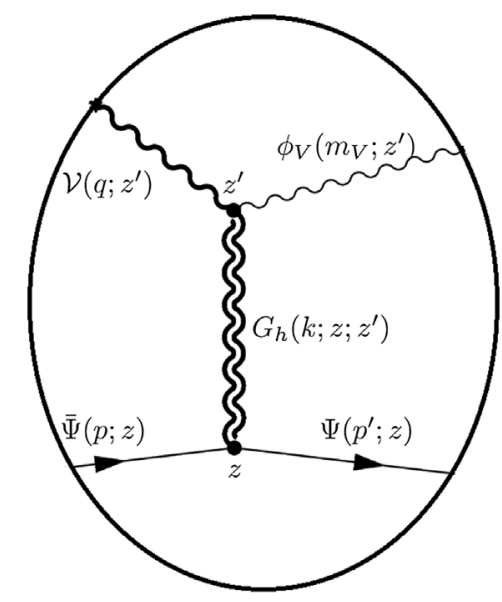

FIG. 2. Witten diagram for the diffractive photoproduction of vector mesons with a bulk wave function $\phi_{V}$. The thick lines or thick wiggles represent the propagators of summed over vector meson or glueball resonances. The thin lines or thin wiggles correspond to a single vector meson and proton. For scalar glueball resonances, due to the dilaton and the trace-full part of the metric fluctuation, we simply replace the bulk-to-bulk propagator $G_{h}\left(k, z, z^{\prime}\right)$ of spin-2 glueballs by $G_{\varphi, f}\left(k, z, z^{\prime}\right)$.

be computed by using the Witten diagram shown in Fig. 2, where bulk VMD is manifest as we will detail below. The structure of the Witten diagram is pretty general, and can be applied to any holographic model to QCD with a mass gap, and a discrete mass spectrum of hadrons.

The main elements of the Witten diagrams shown in Fig. 2 (also in Figs. 2 and 3 for the gravitational form factor) are composed of the following:

(1) The bulk-to-boundary propagator of the vector mesons (or virtual photons for spacelike momenta $\left.q^{2}=-Q^{2}\right)$ as

$$
V(q, z)=\mathcal{V}(q=i Q, z)=C_{V} \times g_{5} \sum_{n} \frac{F_{n} \phi_{n}(z)}{Q^{2}+m_{n}^{2}}
$$

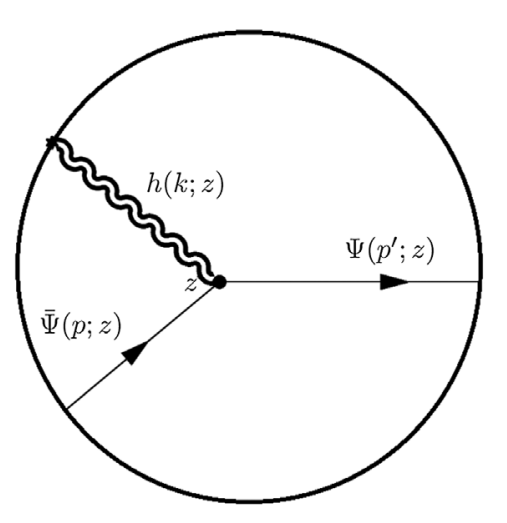

FIG. 3. Witten diagram for the gravitational form factor $A(t)$ due to the exchange of spin-2 glueball resonances. 
where $\phi_{n}\left(m_{n}, z\right), m_{n}, f_{n} \equiv-F_{n} / m_{n}$, and $g_{5}$ are the bulk wave function, mass, decay constant, and hadronic coupling constant of each meson resonances, respectively. $C_{V}$ is a normalization constant for the mesons which can be identified with the value of the electromagnetic form factor of the proton at zeromomentum transfer [and $C_{V}=F_{1}^{(P)}(Q=0)=1$ since the electric charge of the proton is normalized to one in units of $e$.

(2) The bulk-to-boundary propagator of the spin-2 glueballs (for spacelike momenta $k^{2}=-K^{2}$ )

$$
h(k, z)=\mathcal{H}(K, z)=C_{h} \times \sqrt{2} \kappa \sum_{n} \frac{F_{n} \psi_{n}(z)}{K^{2}+m_{n}^{2}},
$$

where $\psi_{n}\left(m_{n}, z\right), m_{n}, f_{n} \equiv-F_{n} / m_{n}$, and $\kappa$ are the bulk wave function, mass, decay constant, and hadronic coupling constant of each glueball resonances. $C_{h}$ is the normalization constant for glueballs [which will be identified with the gravitational form factor of the proton at zero momentum transfer, i.e., $\left.C_{h} \equiv A(t=0)\right]$.

(3) The bulk-to-bulk propagators of the vector meson and glueball resonances

$$
G_{V}\left(q^{\prime}, z, z^{\prime}\right)=C_{V} \times \sum_{n} \frac{\phi_{n}(z) \phi_{n}\left(z^{\prime}\right)}{q^{\prime 2}-m_{n}^{2}}
$$

and

$$
G_{h}\left(k, z, z^{\prime}\right)=C_{h} \times \sum_{n} \frac{\psi_{n}(z) \psi_{n}\left(z^{\prime}\right)}{k^{2}-m_{n}^{2}} ;
$$

and the bulk wave function of the proton (a Dirac fermion in the bulk) is denoted as $\Psi(p, z)$.

More specifically, for the hard-wall and soft-wall holographic models of QCD, that we focus on in this paper, all the ingredients of the Witten diagram Fig. 2 are determined in terms of their bulk wave functions, the normalization constants $C_{V, h}$, the mass scale parameters $z_{0}$ for the hard wall $\left(\tilde{\kappa}_{V, N}\right.$ and $c_{V}$ for the soft wall), and the hadronic coupling constants $g_{5}$ (for mesons) and $\kappa$ (for glueballs).

The mass scale parameters $z_{0}$ or $\tilde{\kappa}_{\rho, N}$ are simultaneously fixed to the proton's and the $\rho$ meson's mass, $\tilde{\kappa}_{V}$ and $c_{V}$ for $V=(J / \psi, \Upsilon)$ are fixed by their mass $m_{V}=\left(m_{J / \psi}, m_{\Upsilon}\right)$ and decay constants $f_{V}=\left(f_{J / \psi}, f_{\Upsilon}\right)$. The hadronic coupling constant of glueballs $\kappa$ is fixed by using type II supergravity action on $\mathrm{AdS}_{5} \times S^{5}$, and the hadronic coupling of vector mesons is fixed by using the DBI action for D7 or D9 flavor branes. Finally, we will extract the gravitational form factor $A(0)=C_{h} / g_{5}^{2}$ by comparing the holographic scattering amplitude to experimental data in the low-energy regime.
Note that, in general, the normalized bulk wave function of one of the vector meson resonances $\phi_{n=0} \equiv \phi_{V}$ takes the form

$$
\phi_{V}=c_{V} z J\left(M_{V} z\right)=\frac{f_{V}}{M_{V}} \times M_{V} z J\left(M_{V} z\right)
$$

where $J\left(M_{V} z\right)$ is a special function that depends on the details of the holographic model. And, the decay constant $f_{V}$, for a meson at rest, defined as

$$
\left\langle 0\left|J_{V, i}\right| V_{j}\right\rangle=f_{V} M_{V} \delta_{i j}
$$

is calculable in a given holographic model to QCD, and can be extracted experimentally from the leptonic width as

$$
\Gamma\left(V \rightarrow \ell^{+} \ell^{-}\right)=\frac{4 \pi}{3} \alpha_{\mathrm{QED}}^{2} e_{V}^{2} \frac{f_{V}^{2}}{M_{V}}
$$

where $e_{V}$ is the electric charge of the constituent quarks of the vector meson. For $V=(J / \Psi, \Upsilon): e_{V}=(2 / 3,1 / 3)$, $M_{V}=(3.097,9.460) \mathrm{GeV}$ and $e_{V} f_{V}=(270,238) \mathrm{MeV}$.

\section{HOLOGRAPHIC MODEL}

We consider $\mathrm{AdS}_{5}$ with a background metric $g_{M N}=$ $\left(\eta_{\mu \nu},-1\right) / z^{2}$ and $\eta_{\mu \nu}=(1,-1,-1,-1)$. Confinement will be described by a background dilaton $\phi=\tilde{\kappa}_{V}^{2} z^{2}$ for mesons, $\phi=\tilde{\kappa}_{N}^{2} z^{2}$ for protons and $\phi=2 \tilde{\kappa}_{N}^{2} z^{2}$ for glueballs in the soft-wall model. In the hard-wall model, $\phi=0$ and confinement is set at $z=z_{0}$. The bulk graviton and dilaton fields will be described by $\varphi$ and $h_{\mu \nu}$ respectively, while the bulk $\mathrm{U}(1)$ vector gauge field and a spin- $\frac{1}{2}$ Dirac fermion by $V^{M}$ and $\Psi$ respectively.

\section{A. Bulk Dirac fermion and vector meson}

The bulk Dirac fermion action in curved $\mathrm{AdS}_{5}$ with minimal coupling to the $\mathrm{U}(1)$ vector meson is [21]

$$
S=\int d^{5} x \sqrt{g}\left(\mathcal{L}_{F}+\mathcal{L}_{V}\right)+\int d^{4} x \sqrt{-g^{(4)}} \mathcal{L}_{\mathrm{UV}}
$$

with the fermionic, gauge field and boundary actions

$$
\begin{aligned}
\mathcal{L}_{F} & =\frac{1}{2 g_{5}^{2}} e^{-\phi(z)}\left(\frac{i}{2} \bar{\Psi} e_{A}^{N} \Gamma^{A}\left(\vec{D}_{N}-\overleftarrow{D}_{N}\right) \Psi-(M+V(z)) \bar{\Psi} \Psi\right) \\
\mathcal{L}_{V} & =-\frac{1}{4 g_{5}^{2}} e^{-\phi(z)} g^{\mu \alpha} g^{\beta \nu} F_{\mu \nu}^{V} F_{\alpha \beta}^{V} \\
\mathcal{L}_{\mathrm{UV}} & =\frac{1}{2 g_{5}^{2}}\left(\bar{\Psi}_{L} \Psi_{R}+\bar{\Psi}_{R} \Psi_{L}\right)_{z=\varepsilon}
\end{aligned}
$$

We have fixed the potential $V(z)=\tilde{\kappa}_{N}^{2} z^{2}$ for both the hardand soft-wall model. We have denoted by $e_{A}^{N}=z \delta_{A}^{N}$ the inverse vielbein, and defined the covariant derivatives 


$$
\begin{aligned}
& \vec{D}_{N}=\vec{\partial}_{N}+\frac{1}{8} \omega_{N A B}\left[\Gamma^{A}, \Gamma^{B}\right]-i V_{N}, \\
& \overleftarrow{D}_{N}=\overleftarrow{\partial}_{N}+\frac{1}{8} \omega_{N A B}\left[\Gamma^{A}, \Gamma^{B}\right]+i V_{N} .
\end{aligned}
$$

The components of the spin connection are $\omega_{\mu z \nu}=-\omega_{\mu \nu z}=$ $\frac{1}{z} \eta_{\mu \nu}$, the Dirac gamma matrices satisfy anticommutation relation $\left\{\Gamma^{A}, \Gamma^{B}\right\}=2 \eta^{A B}$, that is, $\Gamma^{A}=\left(\gamma^{\mu},-i \gamma^{5}\right)$, and $F_{M N}^{V}=$ $\partial_{M} V_{N}-\partial_{N} V_{M}$. The equation of motions for the bulk Dirac fermion and the $\mathrm{U}(1)$ gauge field follow by variation

$$
\begin{aligned}
{\left[i e_{A}^{N} \Gamma^{A} D_{N}-\frac{i}{2}\left(\partial_{N} \phi\right) e_{A}^{N} \Gamma^{A}-(M+\phi(z))\right] \Psi } & =0, \\
\frac{1}{\sqrt{g}} \partial_{M}\left(\sqrt{g} e^{-\phi} F^{M N}\right) & =0 .
\end{aligned}
$$

The coupling $g_{5}$ is inherited from the nature of the brane embeddings in bulk: $1 / g_{5}^{2} \equiv 3 N_{c} N_{f} /\left(12 \pi^{2}\right)$ (D7-branes) and $1 / g_{5}^{2} \equiv\left(3 \sqrt{\lambda} / 2^{5 / 2} \pi\right) N_{c} N_{f} /\left(12 \pi^{2}\right)$ (D9-branes). The brane embeddings with $N_{f}=1$ are more appropriate for describing heavy mesons in bulk, as the U(1) field mode decomposes in an infinite tower of massive vector mesons on these branes as we discussed above. When ignoring these embeddings, the standard assignment is $1 / g_{5}^{2} \equiv N_{c} /\left(12 \pi^{2}\right)$.

We note that in (4.2), we have excluded a Yukawa-type coupling between the dilaton and the bulk Dirac fermion, since neither the fermionic part of the type IIB supergravity action (see, for example, Eq. (A.20) in [22]) nor the fermionic part of the DBI action in string theory (see, for example, Eq. (56) in [23]) support such a coupling.

\section{B. Spectra}

The spectrum for the hard-wall model is fixed by the zeros of the Bessel function $J_{1}\left(m_{n} z_{0}\right)=0$ and does not reggeize. It does in the soft-wall model by solving the equation of motion for $V^{N}$ following from (4.1). The results for the heavy meson masses and decay constants are [24]

$$
\begin{aligned}
m_{n}^{2} & =4 \tilde{\kappa}_{V}^{2}\left(n^{*}+1\right), \\
g_{5} f_{n} & =\sqrt{2} \tilde{\kappa}_{V}\left(\frac{n+1}{n^{*}+1}\right)^{\frac{1}{2}},
\end{aligned}
$$

with $n^{*}=n+c_{V}^{2} / 4 \tilde{\kappa}_{V}^{2}$. The additional constant $c_{V}$ is fixed as $c_{V}^{2} / 4 \tilde{\kappa}_{V}^{2}=M_{V}^{2} / 4 \tilde{\kappa}_{V}^{2}-1$ for $n=0$ for the heavy mesons $V=(J / \psi, \Upsilon)$, and $c_{\rho}=0$ for the light mesons. The mass spectrum of the bulk Dirac fermions is given by [21]

$$
m_{n}^{2}=4 \tilde{\kappa}_{N}^{2}(n+\tau-1),
$$

with the twist factor $\tau$. For the specific soft-wall applications to follow we will set $\tilde{\kappa}_{N}=\tilde{\kappa}_{V}=\tilde{\kappa}_{\rho}$ for simplicity, unless specified otherwise.

\section{Bulk graviton and dilaton}

The graviton in bulk is dual to a glueball on the boundary. It is a rank-2 tensor with reducible parts in general. To decompose the graviton tensor $h_{\mu \nu}$ to its transverse and traceless part $h$, and trace-full part $f$ we follow [25] and define

$$
h_{\mu \nu}=\epsilon_{\mu \nu}^{T T} h+\tilde{k}^{2} \epsilon_{\mu \nu}^{T} f-\tilde{k}_{\mu} \tilde{k}_{\nu} H+\tilde{k}_{\mu} A_{\nu}^{\perp}+\tilde{k}_{\nu} A_{\mu}^{\perp}
$$

where

$$
\begin{aligned}
k^{\mu} \epsilon_{\mu \nu}^{T T} & =\eta^{\mu \nu} \epsilon_{\mu \nu}^{T T}=0, \\
\epsilon_{\mu \nu}^{T} & =\frac{1}{4} \eta_{\mu \nu}
\end{aligned}
$$

with $\alpha \equiv \tilde{k} / k z_{0}$ a dimensionless normalization constant which can be fixed empirically. Here $z_{0}$ is the hard-wall scale, and $k^{\mu} A_{\mu}^{\perp}=0$. A similar rescaling follows in the softwall model with $z_{0} \rightarrow 1 / \tilde{\kappa}_{V}$.

In a gauge where $A_{\mu}^{\perp}=0$, the equation of motion for $h$ decouples. In contrast, the equations for $f, H$, and $\varphi$ (denoted as $k$ in [25]) are coupled (see Eqs. (7.16)-(20) in [25]). Diagonalizing the equations, one can show that $f$ satisfies the same equation of motion as $h$ [25]. Also note that $f_{0}=f(z=0)$ couples to $T_{\mu}^{\mu}$ of the gauge theory, while $H_{0}=H(z=0)$ couples to $k^{\mu} k^{\nu} T_{\mu \nu} \equiv 0$ (see Eq. (7.6) of [25]).

\section{Action}

The effective action for the graviton $\left(\eta_{\mu \nu} \rightarrow \eta_{\mu \nu}+h_{\mu \nu}\right)$ and dilaton fluctuations $(\phi \rightarrow \phi+\varphi)$ follows from the Einstein-Hilbert action plus dilaton by expanding to quadratic order, and after adding the background de-Donder gauge fixing term. The result is

$$
S=\int d^{5} x \sqrt{g} e^{-2 \phi}\left(\mathcal{L}_{h+f}+\mathcal{L}_{\varphi}\right),
$$

with

$$
\begin{aligned}
\mathcal{L}_{h+f} & =-\frac{1}{4 \tilde{g}_{5}^{2}} g^{\mu \nu} \eta^{\lambda \rho} \eta^{\sigma \tau} \partial_{\mu} h_{\lambda \sigma} \partial_{\nu} h_{\rho \tau}+\frac{1}{8 \tilde{g}_{5}^{2}} g^{\mu \nu} \eta^{\alpha \beta} \eta^{\gamma \sigma} \partial_{\mu} h_{\alpha \beta} \partial_{\nu} h_{\gamma \sigma}, \\
\mathcal{L}_{\varphi} & =+\frac{1}{2 \tilde{g}_{5}^{2}} g^{\mu \nu} \partial_{\mu} \varphi \partial_{\nu} \varphi
\end{aligned}
$$

and $\tilde{g}_{5}^{2}=2 \kappa^{2}=16 \pi G_{N}=8 \pi^{2} / N_{c}^{2}$.

\section{Spectrum}

In the soft-wall model, the glueball spectrum is determined by solving the equation of motion for $h_{\mu \nu}$ following from (4.9). The results for the spin-2 glueball masses and decay constants are

$$
m_{n}^{2}=8 \tilde{\kappa}_{N}^{2}(n+1), \quad \tilde{g}_{5} f_{n}=2 \tilde{\kappa}_{N} .
$$

They differ from their vector meson counterparts in (4.5) by the replacements $\tilde{\kappa}_{V} \rightarrow \sqrt{2} \tilde{\kappa}_{N}$ and $g_{5} \rightarrow \tilde{g}_{5}$ due to the difference in the bulk actions. For spin-0 glueballs, we have for the trace-full part of the metric fluctuation 


$$
m_{n}^{2}=8 \tilde{\kappa}_{N}^{2}(n+1), \quad \sqrt{2} \tilde{g}_{5} f_{n}=2 \tilde{\kappa}_{N}
$$

after replacing $\tilde{g}_{5} \rightarrow \sqrt{2} \tilde{g}_{5}$ in the results for spin-2 glueballs. For the dilaton fluctuations we have

$$
m_{n}^{2}=8 \tilde{\kappa}_{N}^{2}(n+1), \quad \tilde{g}_{5} f_{n}=2 \tilde{\kappa}_{N} .
$$

\section{Couplings}

For the graviton in the axial gauge $h_{\mu z}=h_{z z}=0$. The pertinent couplings in Fig. 2, which follow from linearizing the action (4.1) by replacing $\eta_{\mu \nu} \rightarrow \eta_{\mu \nu}+h_{\mu \nu}$, are

$$
\begin{gathered}
h \bar{\Psi} \Psi:-\frac{\sqrt{2 \kappa^{2}}}{2} \int d^{5} x \sqrt{g} h_{\mu \nu} T_{F}^{\mu \nu}, \\
h A A:-\frac{\sqrt{2 \kappa^{2}}}{2} \int d^{5} x \sqrt{g} h_{\mu \nu} T_{V}^{\mu \nu},
\end{gathered}
$$

with the energy-momentum tensors

$$
\begin{aligned}
T_{F}^{\mu \nu}= & e^{-\phi} \frac{i}{2} z \bar{\Psi} \gamma^{\mu} \stackrel{\leftrightarrow}{\partial^{\nu} \Psi}-\eta^{\mu \nu} \mathcal{L}_{F}, \\
T_{V}^{\mu \nu}= & -e^{-\phi}\left(z^{4} \eta^{\rho \sigma} \eta^{\mu \beta} \eta^{\nu \gamma} F_{\beta \rho}^{V} F_{\gamma \sigma}^{V}\right. \\
& \left.-z^{4} \eta^{\mu \beta} \eta^{\nu \gamma} F_{\beta z}^{V} F_{\gamma z}^{V}\right)-\eta^{\mu \nu} \mathcal{L}_{V} .
\end{aligned}
$$

Note that the UV-boundary term in the (4.1) vanishes for the normalizable modes of the fermion. For the dilaton the couplings are

$$
\begin{aligned}
\varphi \bar{\Psi} \Psi: & \sqrt{2 \kappa^{2}} \int d^{5} x \sqrt{g} \frac{e^{-\phi}}{2}\left(\frac{z}{2} \partial_{z} \varphi\right) \bar{\Psi} \gamma^{5} \Psi \\
& +\sqrt{2 \kappa^{2}} \int d^{5} x \sqrt{g} \frac{e^{-\phi}}{2}\left(\frac{i z}{2} \partial_{\mu} \varphi\right) \bar{\Psi} \gamma^{\mu} \Psi, \\
\varphi A A: & \sqrt{2 \kappa^{2}} \int d^{5} x \sqrt{g} e^{-\phi}(-\varphi)\left(-\frac{1}{4} g^{\mu \alpha} g^{\beta \nu} F_{\mu \nu}^{V} F_{\alpha \beta}^{V}\right) .
\end{aligned}
$$

We have canonically normalized the bulk fields through the substitutions

$$
\begin{aligned}
\Psi & \rightarrow g_{5} \Psi, \\
\varphi & \rightarrow \sqrt{2 \kappa^{2}} \varphi, \quad V_{N} \rightarrow g_{5} V_{N}, \\
h_{\mu \nu} & \rightarrow \sqrt{2 \kappa^{2}} h_{\mu \nu}
\end{aligned}
$$

which makes the couplings and power counting manifest in Witten diagrams. Note that after this rescaling, the meson decay constants in (4.5) and the glueball decay constants in (4.11)-(4.13) redefine through $g_{5} f_{n} \rightarrow f_{n}$. This will be understood in most of our analysis.

Evaluating the couplings or the vertices (4.14)-(4.16) on the solutions, Fourier transforming the fields to momentum space, and integrating by part the trace-full part for the fermions, we find for the couplings to the fermions $(h \bar{\Psi} \Psi)$ and gauge fields $(h A A)$

$h \bar{\Psi} \Psi: \int \frac{d^{4} p_{2} d^{4} p_{1} d^{4} k}{(2 \pi)^{12}}(2 \pi)^{4} \delta^{4}\left(p_{2}-k-p_{1}\right)\left(S_{h \bar{\Psi} \Psi}^{k}+S_{f \bar{\Psi} \Psi}^{k}\right)$,

$h A A: \int \frac{d^{4} q^{\prime} d^{4} q d^{4} k}{(2 \pi)^{12}}(2 \pi)^{4} \delta^{4}\left(q^{\prime}-k-q\right)\left(S_{h A A}^{k}+S_{f A A}^{k}\right)$.

The corresponding couplings to the dilatons are

$$
\begin{gathered}
\varphi \bar{\Psi} \Psi: \quad \int \frac{d^{4} p_{2} d^{4} p_{1} d^{4} k}{(2 \pi)^{12}}(2 \pi)^{4} \delta^{4}\left(p_{2}-k-p_{1}\right) S_{\varphi \bar{\Psi} \Psi}^{k}, \\
\varphi A A: \quad \int \frac{d^{4} q^{\prime} d^{4} q d^{4} k}{(2 \pi)^{12}}(2 \pi)^{4} \delta^{4}\left(q^{\prime}-k-q\right) S_{\varphi A A}^{k}
\end{gathered}
$$

with

$$
\begin{aligned}
S_{h \bar{\Psi} \Psi}^{k} & =-\frac{\sqrt{2 \kappa^{2}}}{2} \int d z \sqrt{g} e^{-\phi} z \epsilon_{\mu \nu}^{T T} h(k, z) \bar{\Psi}\left(p_{2}, z\right) \gamma^{\mu} p^{\nu} \Psi\left(p_{1}, z\right), \\
S_{f \bar{\Psi} \Psi}^{k} & =-\frac{\sqrt{2 \kappa^{2}}}{2} \int d z \sqrt{g} e^{-\phi} z \bar{\Psi}\left(p_{2}, z\right)\left(\epsilon_{\mu \nu}^{T} f(k, z) \tilde{k}^{2} \gamma^{\mu} p^{\nu}+\partial_{z}\left(\epsilon_{\mu \nu}^{T} f(k, z)\right) k^{2} \eta^{\mu \nu} \gamma^{5}+\epsilon_{\mu \nu}^{T} f(k, z) \tilde{k}^{2} \eta^{\mu \nu} k_{\alpha} \gamma^{\alpha}\right) \Psi\left(p_{1}, z\right), \\
S_{h A A}^{k} & =\sqrt{2 \kappa^{2}} \int d z \sqrt{g} e^{-\phi} z^{4} \epsilon_{\mu \nu}^{T T} h(k, z) K^{\mu \nu}\left(q, q^{\prime}, n, n^{\prime}, z\right), \\
S_{f A A}^{k} & =\frac{\sqrt{2 \kappa^{2}}}{2} \int d z \sqrt{g} e^{-\phi} z^{4} \epsilon_{\mu \nu}^{T} f(k, z) \tilde{k}^{2}\left(K^{\mu \nu}\left(q, q^{\prime}, n, n^{\prime}, z\right)-\frac{1}{4} \eta^{\mu \nu} K\left(q, q^{\prime}, n, n^{\prime}, z\right)\right),
\end{aligned}
$$

and

$$
\begin{aligned}
S_{\varphi \bar{\Psi} \Psi}^{k} & =\frac{\sqrt{2 \kappa^{2}}}{2} \int d z \sqrt{g} e^{-\phi} z \bar{\Psi}\left(p_{2}, z\right)\left(\partial_{z} \varphi(k, z) \gamma^{5}+\varphi(k, z) k_{\alpha} \gamma^{\alpha}\right) \Psi\left(p_{1}, z\right), \\
S_{\varphi A A}^{k} & =\frac{\sqrt{2 \kappa^{2}}}{4} \int d z \sqrt{g} e^{-\phi} z^{4} \varphi(k, z) K\left(q, q^{\prime}, n, n^{\prime}, z\right) .
\end{aligned}
$$


We have set $q^{2}=-Q^{2}, q^{\prime 2}=-Q^{\prime 2}$ for spacelike momenta, and defined

$$
\begin{aligned}
K^{\mu \nu}\left(q, q^{\prime}, n, n^{\prime}, z\right) \equiv & B_{1}^{\mu \nu} \mathcal{V}(Q, z) \mathcal{V}\left(Q^{\prime}, z\right) \\
& -B_{0}^{\mu \nu} \partial_{z} \mathcal{V}(Q, z) \partial_{z} \mathcal{V}\left(Q^{\prime}, z\right), \\
B_{0}^{\mu \nu}\left(n, n^{\prime}\right) \equiv & n^{\mu} n^{\prime \nu}, \\
B_{1}^{\mu \nu}\left(q, q^{\prime}, n, n^{\prime}\right) \equiv & n \cdot n^{\prime} q^{\mu} q^{\prime \nu}-q \cdot n^{\prime} n^{\mu} q^{\prime \nu} \\
& -q^{\prime} \cdot n q^{\mu} n^{\prime \nu}+q \cdot q^{\prime} n^{\mu} n^{\prime \nu}
\end{aligned}
$$

with $B_{1,0}=\eta_{\mu \nu} B_{1,0}^{\mu \nu}$, and $K=\eta_{\mu \nu} K^{\mu \nu}$. The non-normalizable wave function for the virtual photon $\mathcal{V}(Q, z)$ is given in Appendix A.

\section{GRAVITATIONAL FORM FACTORS}

The graviton coupling to the Dirac fermion in bulk is through its energy-momentum tensor. In the conformally broken geometry (hard or soft wall), the corresponding energy-momentum tensor traces to the normalization of the bulk Dirac fermion as a nucleon state, modulo the source field normalization at the boundary (see below). More importantly, since the holographic construction operates in the limit of a large number of colors, it follows that the energy-momentum tensor of the bulk Dirac fermion is dual

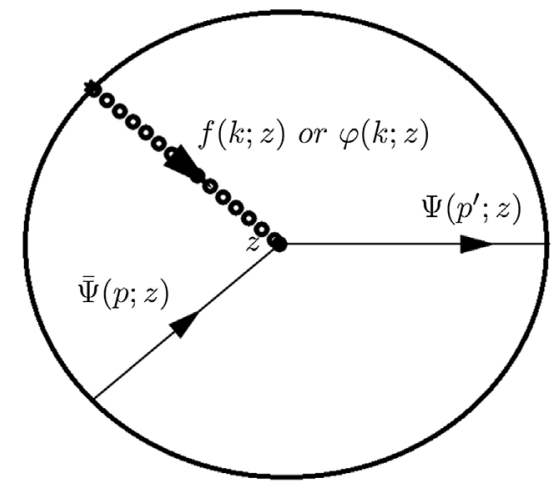

FIG. 4. Witten diagram for the gravitational form factor $C(t)$ due to the exchange of scalar glueball resonances from the tracefull part of the metric fluctuation $f(k, z)$. Also shown is a form factor due to the exchange of the dilatonic scalar glueball resonances $\varphi(k, z)$.

to the quenched energy-momentum tensor of the nucleon. In other words, only the gluonic contribution to the energymomentum tensor is picked by the photoproduction amplitude close to threshold in the present holographic analysis.

More specifically, the energy-momentum tensor to the bulk Dirac fermion involves both the $2^{++}$tensor glueball field $h$ and the $0^{++}$scalar glueball field $f$; see Figs. 3 and 4,

$$
i\left\langle p_{2}\left|T^{\mu \nu}(0)\right| p_{1}\right\rangle=(-i) V_{h \bar{\Psi} \Psi}^{\mu \nu(T T)}\left(p_{1}, p_{2}, K\right)+(-i) V_{f \bar{\Psi} \Psi}^{\mu \nu(T)}\left(p_{1}, p_{2}, K\right),
$$

with the explicit vertices

$$
\begin{aligned}
V_{h \bar{\Psi} \Psi}^{\mu \nu(T T)}\left(p_{1}, p_{2}, K\right)= & -\frac{1}{2 g_{5}^{2}} \int d z \sqrt{g} e^{-\phi} z \bar{\Psi}\left(p_{2}, z\right) \gamma^{\mu} p^{\nu} \Psi\left(p_{1}, z\right) \mathcal{H}(K, z) \\
= & -\frac{1}{2 g_{5}^{2}} \int d z \sqrt{g} e^{-\phi} z\left(\psi_{R}^{2}(z)+\psi_{L}^{2}(z)\right) \mathcal{H}(K, z) \times \bar{u}\left(p_{2}\right) \gamma^{\mu} p^{\nu} u\left(p_{1}\right), \\
V_{f \bar{\Psi} \Psi}^{\mu \nu(T)}\left(p_{1}, p_{2}, K\right)= & -\frac{1}{2 g_{5}^{2}} \int d z \sqrt{g} e^{-\phi} z\left(\psi_{L}(z) \psi_{R}(z)-\psi_{R}(z) \psi_{L}(z)\right) \partial_{z} \mathcal{F}(K, z) \times \tilde{k}^{2} \eta^{\mu \nu} \times \bar{u}\left(p_{2}\right) u\left(p_{1}\right) \\
& -\frac{1}{16 g_{5}^{2}} \int d z \sqrt{g} e^{-\phi} z\left(\psi_{R}^{2}(z)+\psi_{L}^{2}(z)\right) \mathcal{F}(K, z) \times \tilde{k}^{2} \eta^{\mu \nu} \times \bar{u}\left(p_{2}\right)\left(\gamma_{\alpha} p^{\alpha}+4 k_{\alpha} \gamma^{\alpha}\right) u\left(p_{1}\right) .
\end{aligned}
$$

They follow by substituting the normalizable mode $J_{h}\left(m_{n}, z\right)$ and $J_{f}\left(m_{n}, z\right)$ by the non-normalizable mode $\mathcal{H}(K, z)$ (given in (A31) and $\mathcal{F}(K, z)$ (given in (A49) in the second vertices of (B6) and (B7) for spacelike momenta $k^{2}=-K^{2}$, with the boundary value for the source set generically to $\mathcal{H}(K, 0)=1$. Below, we show that this boundary condition is tied to the normalization of the (gluonic) trace of the energy-momentum tensor in the bulk Dirac fermion state as a nucleon and will relax it, since it is arbitrary in holography.

With this in mind, a comparison of (5.1)-(5.3) to the standard decomposition of the energy-momentum form factor

$$
\begin{aligned}
\left\langle p_{2}\left|T^{\mu \nu}(0)\right| p_{1}\right\rangle= & \bar{u}\left(p_{2}\right)\left(A(k) \gamma^{(\mu} p^{\nu)}+B(k) \frac{i p^{(\mu} \sigma^{\nu) \alpha} k_{\alpha}}{2 m_{N}}\right. \\
& \left.+C(k) \frac{k^{\mu} k^{\nu}-\eta^{\mu \nu} k^{2}}{m_{N}}\right) u\left(p_{1}\right)
\end{aligned}
$$

yields

$$
\begin{aligned}
A(K) & =-\frac{C(K)}{\left(\alpha z_{0} m_{N} / 2\right)^{2}} \\
& =\frac{1}{2 g_{5}^{2}} \int d z \sqrt{g} e^{-\phi} z\left(\psi_{R}^{2}(z)+\psi_{L}^{2}(z)\right) \mathcal{H}(K, z) .
\end{aligned}
$$


For the soft-wall model,

$$
\begin{aligned}
A(K)= & A(0)\left(a_{K}+1\right)\left(-\left(1+a_{K}+2 a_{K}^{2}\right)\right. \\
& \left.+2\left(a_{K}+2 a_{K}^{3}\right) \Phi\left(-1,1, a_{K}\right)\right),
\end{aligned}
$$

or equivalently

$$
\begin{aligned}
A(K)= & A(0)\left(\left(1-2 a_{K}\right)\left(1+a_{K}^{2}\right)\right. \\
& \left.+a_{K}\left(1+a_{K}\right)\left(1+2 a_{K}^{2}\right)\left(\psi\left(\frac{1+a_{K}}{2}\right)-\psi\left(\frac{a_{K}}{2}\right)\right)\right)
\end{aligned}
$$

with $a_{K}=K^{2} / 8 \tilde{\kappa}_{N}^{2}$. Here $\Phi\left(-1,1, a^{\prime}\right)$ refers to the LerchPhi function, and $\psi(x)$ refers to the digamma function or harmonic number $H_{x}=\psi(x)+\gamma$. Modulo $A(0),(5.5)$ is in agreement with the result in [21]. The gravitational form factor $C(K)$ is proportional to $A(K)$ modulo a negative overall constant $-\left(\alpha z_{0} m_{N} / 2\right)^{2}<0$ which is left undetermined since $\alpha$ is arbitrary in the tensor decomposition (4.7). We note that (5.1) gives $\left\langle p\left|T_{\mu}^{\mu}\right| p\right\rangle=2 A(0) m_{N}^{2}$. Since the boundary value $\mathcal{H}(K, 0)=\mathcal{H}(0, z)$ is arbitrary as we just noted above, it follows that $A(0)$ is not fixed in holography. This will be understood from here on.

The invariant form factors $A(k), B(k), C(k)$ (for $k^{2} \geq 0$ ) measure the gluonic content of the energy-momentum tensor in the nucleon state, as the holographic dual of the energy-momentum tensor of the dilation in bulk in the double limit of large $N_{c}, \lambda$. This limit maps the bulk fields in a soft- or hard-wall metric, to a pure Yang-Mills theory at the boundary in the confining regime. More specifically, the form factor $A(k)$ through $\mathcal{H}(K, z)$ in bulk resums the $2^{++}$or tensor glueball Regge trajectory as given in (A31). For the soft-wall model, the result is in agreement with the one reported in [21]. The form factor $C(k)$ through $\mathcal{F}(K, z)$ in bulk resums the $0^{++}$or scalar glueball Regge trajectory as shown in (A49). In holography, the scalar and tensor glueball spectra are degenerate as we noted earlier (same bulk equations for $h, f)$, so $\mathcal{H}(K, z)$ and $\mathcal{F}(K, z)$ are tied, i.e., $\mathcal{F}=-2 \mathcal{H}$. The factor of 2 reflects on the $\frac{1}{2}$ difference in the normalization of the kinetic energies in (4.10). Finally, the Pauli-like form factor $B(k)=0$ as the coupling of the graviton to the bulk Dirac fermion through the spin connection in (4.2) vanishes,

$$
\frac{1}{8} \omega_{N A B} \bar{\Psi} e_{C}^{N} \Gamma^{C} \frac{i}{2}\left[\Gamma^{A}, \Gamma^{B}\right] \Psi \rightarrow \frac{i}{8} h_{\alpha}^{\mu} \bar{\Psi} \Gamma^{\alpha}\left[\Gamma_{\mu}, \Gamma^{z}\right] \Psi=0 .
$$

The soft-walll results for the gravitational form factor $A(k)$ compares well with the recently reported lattice results, as shown in Fig. 5. The solid blue curve is our result for the soft-wall model, and the red squares are the recent lattice data [26]. The resummed $A(k)$ (for $k^{2} \geq 0$ ) in

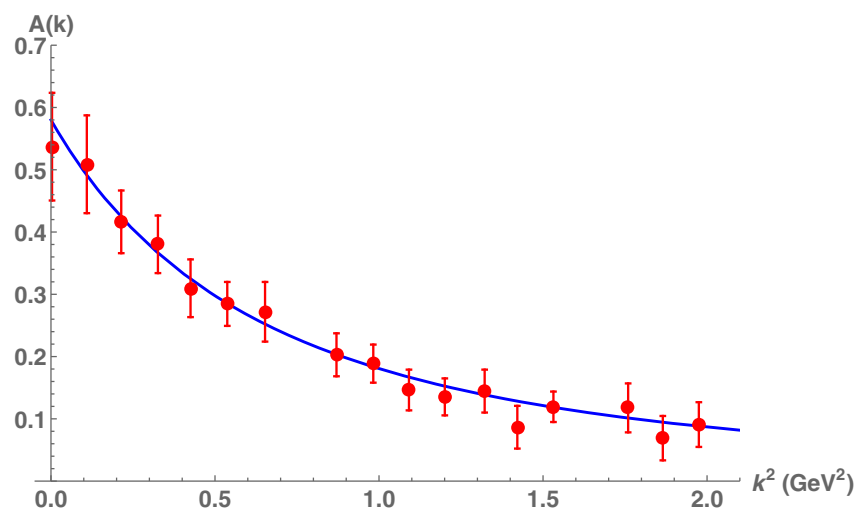

FIG. 5. Holographic gravitational form factor $A(k)$ (for $k^{2} \geq 0$ ) shown in solid blue curve versus the lattice data in red squares [26].

the soft-wall model is well reproduced by the dipole form factor

$$
A(k)=\frac{A(0)}{\left(1+\frac{k^{2}}{m_{A}^{2}}\right)^{2}}
$$

with $m_{A}=1.124 \mathrm{GeV}$ in comparison to the reported lattice value $m_{A, \text { lattice }}=1.13 \mathrm{GeV}$. The arbitrary normalization $A(0)=0.58$ was adjusted to the lattice data [26]. Recall that the gravitational form factor $A(k)$ is saturated by the $2^{++}$glueball trajectory without any quark mixing, essentially a quenched result. In Fig. 6 we show in the solid blue curve the holographic gravitational form factor $D(k) \equiv$ $4 C(k)=-4 A(k)$ with $\alpha=2 /\left(z_{0} m_{N}\right)$ in the soft-wall model, versus the reported lattice results in red squares [26]. In holography $C(k)$ is saturated by the $0^{++}$massive glueballs which are degenerate with the $2^{++}$ones, hence $m_{A}=1.124 \mathrm{GeV}$ in comparison to $m_{A}=0.48 \mathrm{GeV}$ from the lattice. The difference is likely due to the strong scalarisoscalar quark mixing to the $0^{++}$gueball channel in the unquenched lattice simulations, in particular to the light sigma meson with a mass of about $0.5 \mathrm{GeV}$. In Fig. 7 we

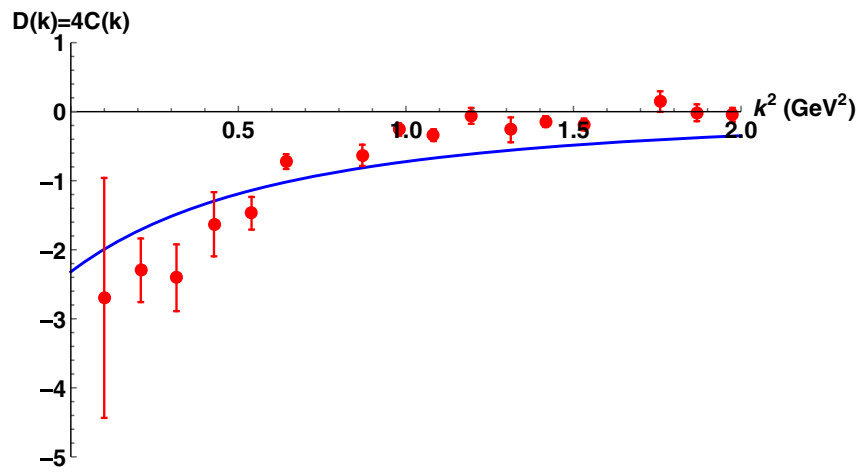

FIG. 6. Holographic gravitational form factor $D(k)=4 C(k)$ (for $k^{2} \geq 0$ ) shown in solid blue curve versus the lattice data in red squares [26]. 


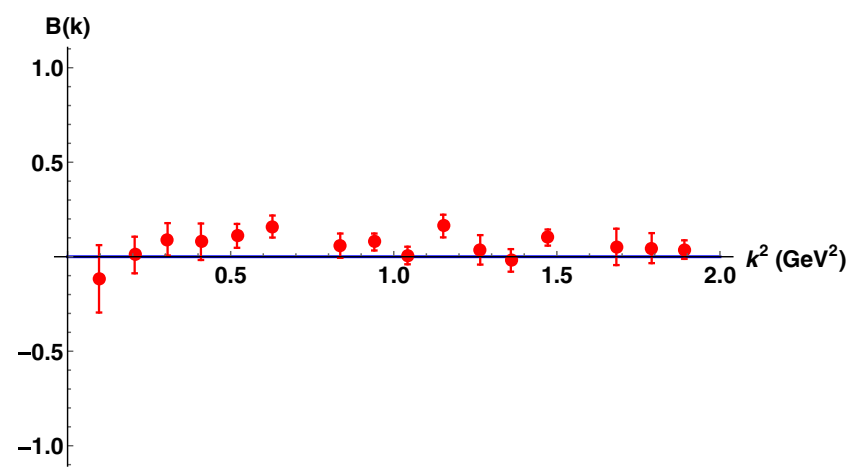

FIG. 7. Holographic gravitational form factor $B(k)=0$ (for $k^{2} \geq 0$ ) shown in solid blue curve versus the lattice data in red squares [26].

show the lattice results in red squares for $B(k)$ which are consistent with $B(k)=0$ in holography shown as a solid blue curve.

\section{HOLOGRAPHIC PRESSURE AND SHEAR INSIDE THE PROTON}

Using the dipole representation for $A(K)(5.8)$ which is a good parametrization of our holographic results, the D-term as $D(K)=-4 A(K)$ can be written as

$$
D(K)=\frac{-4 A(0)}{\left(1+\frac{K^{2}}{m_{A}^{2}}\right)^{2}}
$$

with $m_{A}=1.124 \mathrm{GeV}$. The Fourier transform (6.1) of the three-dimensional coordinate space gives $\left(E=m_{N}\right)$

$\tilde{D}(r)=-4 A(0) \int \frac{d^{3} K}{2 E(2 \pi)^{3}} \frac{e^{-i K \cdot r}}{\left(1+\frac{K^{2}}{m_{A}^{2}}\right)^{2}}=-A(0) \frac{m_{A}^{3}}{4 \pi m_{N}} e^{-m_{A} r}$.

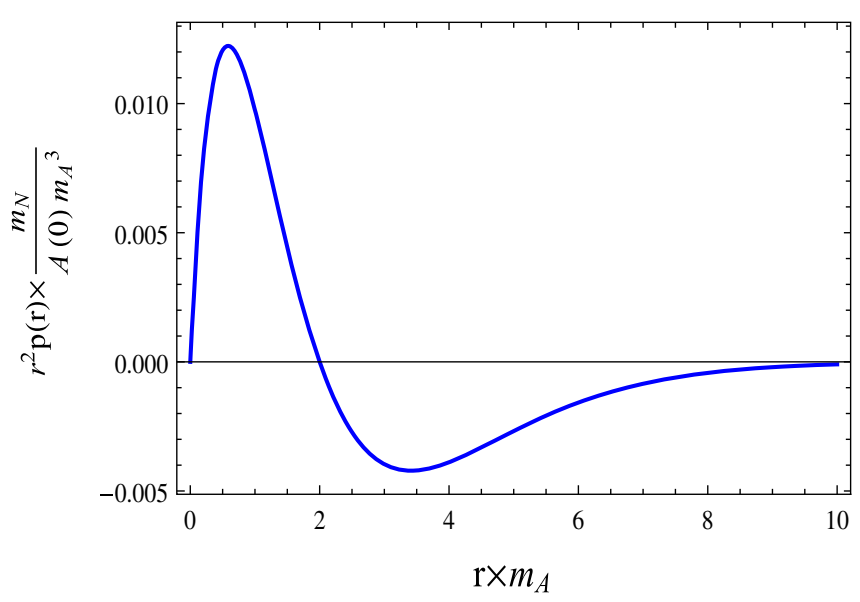

(a) The pressure distribution inside the proton (VI.47) for soft-wall holographic QCD with $m_{A}=1.124 \mathrm{GeV}$.
The holographic shear $s(r)$ and pressure $p(r)$ distributions in the proton can be expressed in terms of $\tilde{D}(r)$ as [27]

$$
\begin{aligned}
& s(r)=-\frac{r}{2} \frac{d}{d r} \frac{1}{r} \frac{d}{d r} \tilde{D}(r), \\
& p(r)=\frac{1}{3} \frac{1}{r^{2}} \frac{d}{d r} r^{2} \frac{d}{d r} \tilde{D}(r) .
\end{aligned}
$$

In Figs. 8(a) and 8(b) we show the holographic gluon contribution to the pressure $p(r)$ distribution and shear force $s(r)$, respectively. The results are in agreement with the lattice QCD result [28] for the gluon contribution. They are also comparable to the experimentally extracted quark contributions in [29]. Below, we will argue that the holographic relationship $D(K)=-4 A(K)$ will allow the extraction of the pressure and shear of the proton from the threshold photoproduction data of heavy vector mesons $V=J / \Psi, \Upsilon$.

\section{HOLOGRAPHIC VECTOR MESON DOMINANCE}

The diffractive scattering amplitude with a single graviton and dilaton exchanges is detailed in Appendix B. For photoproduction or electroproduction close to the photon point $Q^{2}=0$, and we may set $\mathcal{V}(Q=0, z)=1$ in $\mathcal{V}_{h A A}$ in (B9). This will be indicated by the relabeling of the entry photon $A \rightarrow \gamma$. This will be understood in the remainder of our analysis. With this in mind, the combined amplitudes (B4) read

$$
\begin{aligned}
-\mathcal{A}_{\gamma p \rightarrow A p}(s, t)= & \mathcal{A}_{\gamma p \rightarrow A p}^{\varphi}(s, t)+\mathcal{A}_{\gamma p \rightarrow A p}^{h}(s, t)+\mathcal{A}_{\gamma p \rightarrow A p}^{f}(s, t) \\
= & \frac{1}{2 g_{5}^{4}} \mathcal{V}_{h A A} B_{\alpha \beta}^{1} \mathcal{V}_{h \bar{\Psi} \Psi}^{\alpha \beta(T T)}+\frac{1}{g_{5}^{4}} \mathcal{V}_{f A A} B^{1} \mathcal{V}_{f \bar{\Psi} \Psi}^{(T)} \\
& +\frac{1}{g_{5}^{4}} \tilde{\mathcal{V}}_{\varphi A A} B^{1} \mathcal{V}_{\varphi \bar{\Psi} \Psi}
\end{aligned}
$$

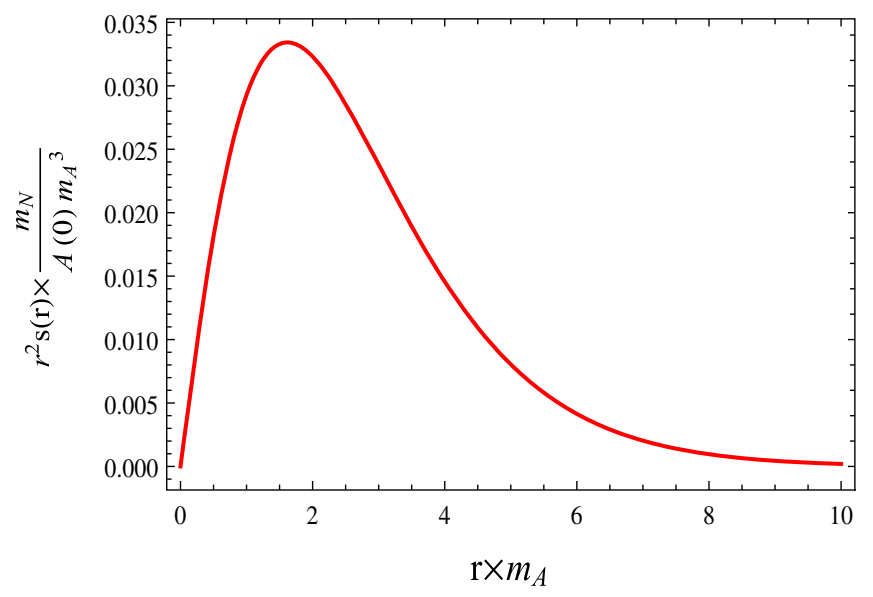

(b) The shear force inside the proton (VI.47) for soft-wall holographic QCD with $m_{A}=1.124 \mathrm{GeV}$.

FIG. 8. Holographic pressure and shear inside the proton. 
The effective vertices for the hard-wall model are

$$
\begin{aligned}
& \mathcal{V}_{h A A}=\frac{\sqrt{2 \kappa^{2}}}{2} \int_{0}^{z_{0}} d z \sqrt{g} z^{4} \mathcal{V}\left(Q^{\prime}, z\right) \frac{z^{4}}{4}, \\
& \mathcal{V}_{f A A}=\eta_{\mu \nu} \mathcal{V}_{f A A}^{\mu \nu(T)}=0, \\
& \tilde{\mathcal{V}}_{\varphi A A}=\frac{\sqrt{2 \kappa^{2}}}{4} \int_{0}^{z_{0}} d z \sqrt{g} z^{4} \mathcal{V}\left(Q^{\prime}, z\right) \frac{z^{4}}{4}, \\
& \mathcal{V}_{\varphi \bar{\Psi} \Psi}=0 .
\end{aligned}
$$

The corresponding vertices for the soft-wall model follows through the substitution $\sqrt{g} \rightarrow \sqrt{g} e^{-\tilde{\kappa}_{V}^{2} z^{2}}$ with $\tilde{\kappa}_{V}$ the softwall scale. $B_{\alpha \beta}^{1}$ and $B^{1}=\eta^{\mu \nu} B_{\mu \nu}^{1}$ are defined in (4.22).

The TT-part of the transverse and traceles $2^{++}$glueball contribution corresponds to $\alpha, \beta=x, y$. The T-coupling of the transverse and trace-full $0^{++}$glueball to the virtual photons involves the full photon energy-momentum tensor and vanishes after contraction with $\epsilon_{\mu \nu}^{T}$. The TT-coupling involves only the nontrace part of the photon energymomentum tensor and does not vanish after contraction with $\epsilon_{\mu \nu}^{T T}$. The Yukawa coupling of the dilaton to the bulk
Dirac fermion is null as we noted earlier. As a result, the scattering amplitude (7.1) is solely due to the exchange of the $2^{++}$glueball.

The result (7.1)-(7.3) is for a general bulk-to-boundary current $\mathcal{V}\left(Q^{\prime}, z\right)$ which sums over a tower of vector meson resonances. The production of a specific meson at the boundary, say charmonium or upsilonium, amounts to the substitution

$\mathcal{V}\left(Q^{\prime}, z\right) \rightarrow \phi_{n}(z)=c_{n} z J_{1}\left(m_{n} z\right)=\frac{f_{n}}{m_{n}}\left(m_{n} z\right) J_{1}\left(m_{n} z\right)$

in (7.2)-(7.2) with $c_{n}=\frac{\sqrt{2}}{z_{0} J_{1}\left(m_{n} z_{0}\right)}$ and $f_{n}$ the decay constant of a heavy meson of mass $m_{n}$, with the identification $n=0$ for $J / \Psi$. As a result, the total amplitude for the photoproduction of $J / \Psi$ can be written in the block form

$$
\mathcal{A}_{\gamma p \rightarrow J / \Psi p}(s, t)=-\frac{1}{2 g_{5}^{4}} \mathcal{V}_{h A A} B_{\alpha \beta}^{1} \mathcal{V}_{h \bar{\Psi} \Psi}^{\alpha \beta(T T)}
$$

with the vertices for a hard wall

$$
\begin{aligned}
V_{h \bar{\Psi} \Psi}^{\mu \nu}\left(p_{1}, p_{2}, K\right) & =-\frac{\sqrt{2 \kappa^{2}}}{2} \int d z \sqrt{g} e^{-\phi} z\left(\psi_{R}^{2}(z)+\psi_{L}^{2}(z)\right) \mathcal{H}(K, z) \times \bar{u}\left(p_{2}\right) \gamma^{\mu} p^{\nu} u\left(p_{1}\right) \\
\mathcal{V}_{h A A} & =\left(\frac{f_{n}}{m_{n}}\right) \times \frac{\sqrt{2 \kappa^{2}}}{2} \int_{0}^{z_{0}} d z \sqrt{g} z^{4} \times\left(m_{n} z\right) J_{1}\left(m_{n} z\right) \times \frac{z^{4}}{4} \\
& \approx\left(\frac{f_{n}}{m_{n}}\right) \times\left(\frac{\sqrt{2 \kappa^{2}}}{16 m_{n}^{4}} \int_{0}^{w_{0}} d w w^{5}\right) \equiv\left(\frac{f_{V}}{M_{V}}\right) \mathbb{V}_{h A A},
\end{aligned}
$$

with $w=m_{n} z, w_{0}=m_{n} z_{0}$. The wave function for the emitted meson near the boundary is $J_{1}(w) \approx w / 2$. In comparison, the same arguments for the soft-wall model give

$$
\begin{aligned}
\mathcal{V}_{h A A} & =\left(\frac{f_{n}}{m_{n}}\right) \times \frac{\sqrt{2 \kappa^{2}}}{2} \int_{0}^{\infty} d z \sqrt{g} e^{-z^{2} \tilde{\kappa}_{V}^{2}} z^{4} \times\left(2 \tilde{\kappa}_{V}^{2} z^{2}\right) L_{n}^{1}\left(z^{2} \tilde{\kappa}_{V}^{2}\right) \times \frac{z^{4}}{4} \\
& \approx\left(\frac{f_{n}}{m_{n}}\right) \times\left(\frac{\sqrt{2 \kappa^{2}}}{2} \frac{L_{n}^{1}(0)}{4 \tilde{\kappa}_{V}^{4}} \int_{0}^{\infty} d \xi e^{-\xi^{2}} \xi^{2}\right) \equiv\left(\frac{f_{V}}{M_{V}}\right) \mathbb{V}_{h A A},
\end{aligned}
$$

with $\xi=\tilde{\kappa}_{V}^{2} z^{2}$ and $\tilde{\kappa}_{V}$ the soft-wall parameter, and $n=0$.

Equations (7.6) and (7.7) embody the general strictures of VMD with the emergence of $f_{n} / m_{n} \equiv f_{V} / M_{V}$, the ratio of the leptonic decay constant to the mass of the heavy meson emitted, as illustrated in Fig. 2. This result shows that in holographic QCD, the photoproduction amplitude $\gamma p \rightarrow V p$ follows from the inverse of the diffractive part of the deeply virtual Compton scattering amplitude $\gamma^{*} p \rightarrow \gamma p$ through VMD with $\gamma^{*} \approx\left(e f_{V} / M_{V}\right) V$.

The triple coupling $\mathbb{V}_{h A A}$ is the coupling of the bulk graviton with wave function near the boundary $h \approx$ $z^{2} J_{2}\left(m_{n} z\right) \approx z^{4}$ (heavy $2^{++}$glueball), to a virtual photon near mass shell with $\mathcal{V}(Q \approx 0, z) \rightarrow 1$, and a virtual photon off mass shell with $\mathcal{V}\left(Q^{\prime}, z\right) \rightarrow\left(f_{n} / m_{n}\right) \times\left(m_{n} z\right) J_{1}\left(m_{n} z\right)$ (hard wall) or $\mathcal{V}\left(Q^{\prime}, z\right) \rightarrow\left(f_{n} / m_{n}\right) \times\left(2 \tilde{\kappa}_{V}^{2} z^{2}\right) L_{n}^{1}\left(z^{2} \tilde{\kappa}^{2}\right)$ (soft wall). The masses and decay constant for the soft wall are given in (4.5) with the proviso that $g_{5} f_{n} \rightarrow f_{n}$ following the canonical rescaling (4.17).

\section{DIFFERENTIAL CROSS SECTION FOR PHOTOPRODUCTION}

Although our analysis for vector meson production applies equally well to both photoproduction and electroproduction, 
we now specialize to the photoproduction of heavy mesons given the recent experimental interest in extracting the gluon contribution to the proton state from threshold data at current electron machine facilities. With this in mind, the differential cross section for photoproduction of $V=J / \Psi$ can now be constructed from leading spin $j=0,2$ glueball exchanges near threshold. The contribution of higher spin-j exchanges and their reggeization will follow. The pertinent differential cross section is of the form

$$
\left(\frac{d \sigma}{d t}\right)=\frac{e^{2}}{16 \pi\left(s-m_{N}^{2}\right)^{2}} \frac{1}{2} \sum_{\mathrm{pol}} \frac{1}{2} \sum_{\text {spin }}\left|\mathcal{A}_{\gamma p \rightarrow J / \Psi p}^{h}(s, t)\right|^{2},
$$

which is dominated by the TT-part of the graviton or $2^{++}$glueball exchange as we noted earlier. The first sum over the photon and $J / \Psi$ polarizations is carried out using the identities

$$
\begin{aligned}
\sum_{s=1,2} n_{s}^{\mu} n_{s}^{* \nu} & =-\eta^{\mu \nu}, \\
\sum_{s^{\prime}=1,2,3} n_{s^{\prime}}^{\prime \prime} n_{s^{\prime}}^{\prime * \nu} & =-\eta^{\mu \nu}+\frac{q^{\prime \mu} q^{\prime \nu}}{M_{V}^{2}} .
\end{aligned}
$$

The second sum is over the initial and final bulk Dirac fermion as a proton spin

$$
\frac{1}{4} \operatorname{Tr}\left(\left(\gamma_{\mu} p_{2}^{\mu}+m_{N}\right)\left(\gamma_{\mu} p_{1}^{\mu}+m_{N}\right)\right)=2 K^{2}+8 m_{N}^{2} .
$$

Carrying explicitly these summations yield the differential cross section for photoproduction of a heavy meson in the spin $j=2$ exchange approximation as

$$
\begin{aligned}
\left(\frac{d \sigma}{d t}\right)= & \frac{e^{2}}{64 \pi\left(s-m_{N}^{2}\right)^{2}} \times\left(\frac{f_{V}}{M_{V}}\right)^{2} \mathbb{V}_{h A A}^{2} \times \frac{\kappa^{2}}{2 g_{5}^{8}} \\
& \times \frac{g_{5}^{4} A^{2}(K)}{m_{N}^{2}} \times F\left(s, t=-K^{2}, M_{V}, m_{N}\right) \\
& \times\left(2 K^{2}+8 m_{N}^{2}\right), \\
= & \mathcal{N}^{2} \times \frac{e^{2}}{64 \pi\left(s-m_{N}^{2}\right)^{2}} \times \frac{A^{2}(K)}{4 m_{N}^{2} A^{2}(0)} \\
& \times F\left(s, t=-K^{2}, M_{V}, m_{N}\right) \times\left(2 K^{2}+8 m_{N}^{2}\right)
\end{aligned}
$$

with all vertex insertions following the rescaling (4.17) are shown explicitly and, in the last line, we have defined the normalization factor $\mathcal{N}$ as

$$
\mathcal{N}^{2}=\left(\frac{f_{V}}{M_{V}}\right)^{2} \mathbb{V}_{h A A}^{2} \times \frac{2 \kappa^{2}}{g_{5}^{8}} \times g_{5}^{4} A^{2}(0),
$$

where $A(K)$ is the gravitational form factor (5.4), which reduces to (5.5) for the soft-wall model. The kinematical function $F\left(s, t, M_{V}, m_{N}\right)$ follows from the contractions of the various spins emanating from the photon and graviton vertices, and reads

$$
\begin{aligned}
F(s, t, M, m)= & \frac{1}{4096 M^{2}}\left[-9 M^{10}+M^{8}\left(-32+68 m^{2}+28 s+37 t\right)+2 M^{6}\left(256 m^{4}+8 m^{2}(32 s-3 t)+t(56-40 s-29 t)\right)\right. \\
& +2 M^{4}\left(-136 m^{6}+64 s^{2}-56 s^{3}+8 m^{4}(8+27 s-64 t)+3 t^{2}(-24+7 t)+4 s t(-4+9 t)-4 m^{2}\left(6 s^{2}+32 s(1+4 t)\right.\right. \\
& +t(-4+25 t)))+M^{2}\left(144 m^{8}+144 s^{4}-192 s^{2} t+96 s^{3} t-16 s(-4+t) t^{2}+(80-13 t) t^{3}+96 m^{6}(-6 s+7 t)\right. \\
& \left.+32 m^{4}\left(27 s^{2}-6 t-39 s t+8 t^{2}\right)+16 m^{2}\left(-36 s^{3}+30 s^{2} t+24 s t(1+2 t)+t^{2}(-4+17 t)\right)\right) \\
& -t\left(2 m^{2}-2 s-t\right)\left(64 m^{4}+8 m^{6}-8 s^{3}+76 m^{4} t-16 t^{2}-90 m^{2} t^{2}+t^{3}+4 s^{2}\left(16+6 m^{2}+3 t\right)\right. \\
& \left.\left.-2 s\left(12 m^{4}+3 t^{2}+m^{2}(64+44 t)\right)\right)\right]
\end{aligned}
$$

with $M_{V}=M, m \equiv m_{N}$, and $V=J / \Psi$, . In the double limit of large $N_{c}, \lambda$, the differential cross section (8.4) scales as

$$
\begin{aligned}
\frac{d \sigma}{d t} & \sim f_{V}^{2}\left(\frac{\kappa^{4}}{g_{5}^{4}}\right) \\
& \sim \frac{1}{N_{c}^{0}}\left(\lambda^{0}: \text { soft wall; } \lambda^{0}: \text { D7 brane; } \lambda^{2}: \text { D9 brane; }\right)
\end{aligned}
$$

since $f_{V} \sim N_{c}^{0}$ after the rescaling (4.17). It differs from the scaling of the surface exchange in [5], where their bulk Dirac fermion action is not normalized with $1 / g_{5}^{2}$. For large $s$, we note that $F(s, t) \sim s^{4}$ and the differential cross section is seen to grow like $s^{2}$ as expected from a $2^{++}$glueball exchange as a graviton. The corresponding amplitude is purely real in this limit. These features reflect on the shortcomings of the $j=2$ exchange and its lack of reggeization at large $\sqrt{s}$. They will be addressed below.

This notwithstanding, the differential cross section for photoproduction of a heavy meson is proportional to the gravitational form factor $A(K)$ with $A(0)$ the sought after gluonic contribution to the trace of the energy-momentum tensor. However, it is folded with various couplings and kinematical factors that makes its extraction at threshold challenging. For the numerical analysis to follow, we will 


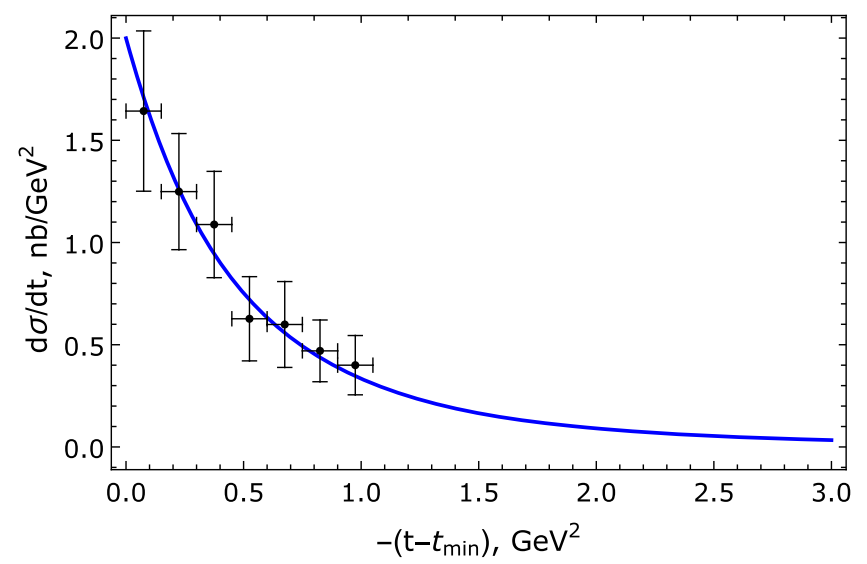

FIG. 9. Differential cross section for $V=J / \Psi$ photoproduction for $E_{\gamma}=10.72 \mathrm{GeV}$. The solid blue curve is our result for the soft-wall model. The data near threshold are from GlueX [15].

use the soft-wall model with a fixed scale $\tilde{\kappa}_{N}=0.350 \mathrm{GeV}$, $\kappa^{2}=4 \pi^{2} / N_{c}^{2}$ as fixed by the normalization of the kinetic part of the gravitational action in (4.10), and set $1 / g_{5}^{2}$ through the D7 or D9 brane embeddings. The coupling $\mathbb{V}_{h A A}$ is fixed by setting $V=J / \Psi$ in bulk.

In Fig. 9, we show the behavior of the differential cross section (8.4) for $V=J / \Psi$ photoproduction for a photon energy $E_{\gamma}=10.72 \mathrm{GeV}$ in comparison to the GlueX recent data near threshold [15]. The solid blue curve is our result for the soft-wall model. The data near threshold are from GlueX [15]. The mesonic parameters were fixed using (4.5) with $m_{0}=M_{J / \psi}=3.10 \mathrm{GeV}$ and $f_{0}=f_{J / \psi}=$ $0.405 \mathrm{GeV}$ for the soft-wall model, and

$$
\begin{aligned}
& \tilde{\kappa}_{V}=\frac{2^{3 / 8} \pi^{3 / 4}}{3^{1 / 4}} \frac{\sqrt{f_{V} m_{V}}}{\lambda^{1 / 8}} \quad(\mathrm{D} 9 \text { model }), \\
& \tilde{\kappa}_{V}=\frac{2^{3 / 8} \pi^{3 / 4}}{3^{1 / 4}} \frac{\sqrt{f_{V} m_{V}}}{\left(2^{5 / 2} \pi\right)^{1 / 4}} \quad(\mathrm{D} 7 \text { model) }, \\
& \tilde{\kappa}_{V}=\frac{2^{3 / 8} \pi^{3 / 4}}{3^{1 / 4}} \frac{\sqrt{f_{V} m_{V}}}{\left(2^{5 / 2} \pi / 3\right)^{1 / 4}} \quad \text { (original). }
\end{aligned}
$$

Here the label "original" refers to the original soft-wall model. The value of the form factor $A(0)$ is model dependent and follows from fitting the normalization factor $\mathcal{N}$, defined in (8.5), to data as

$$
\begin{aligned}
& \mathcal{N}=\frac{20 \sqrt{\lambda} N_{f} A(0)}{\left(10 \tilde{\kappa}_{V}\right)^{4}}=\sqrt{\frac{f_{J / \psi} m_{J / \psi}}{f_{V} m_{V}}} \times 7.768 \mathrm{GeV}^{-4} \quad(\mathrm{D} 9 \text { model }) \\
& \mathcal{N}=\frac{202^{5 / 2} \pi \times N_{f} A(0)}{\left(10 \tilde{\kappa}_{V}\right)^{4}}=\sqrt{\frac{f_{J / \psi} m_{J / \psi}}{f_{V} m_{V}}} \times 7.768 \mathrm{GeV}^{-4} \quad(\mathrm{D} 7 \text { model }), \\
& \mathcal{N}=\frac{202^{5 / 2} \pi \times N_{f} A(0)}{3\left(10 \tilde{\kappa}_{V}\right)^{4}}=\sqrt{\frac{f_{J / \psi} m_{J / \psi}}{f_{V} m_{V}}} \times 7.768 \mathrm{GeV}^{-4} \quad \text { (original) } .
\end{aligned}
$$

In Fig. 10 we show how changing the overall normalization (8.9) affects the result in Fig. 9. The blue band corresponds to $7.768 \rightarrow 7.768 \pm 0.732$ in (8.9) which would amount to about a $\pm 10 \%$ change in $A(0)$ for fixed hologaphic parameters, and vice versa.

In Fig. 11 we show the same differential cross section for other photon energies in dashed red in comparison to $E_{\gamma}=$ $10.72 \mathrm{GeV}$ in solid blue and the GlueX recent data [15]. The large red dashed curve is for $E_{\gamma}=11 \mathrm{GeV}$, the medium red dashed curve is for $E_{\gamma}=10.6 \mathrm{GeV}$, the small red dashed curve is for $E_{\gamma}=10.3 \mathrm{GeV}$ and the dotted red curve is for $E_{\gamma}=10 \mathrm{GeV}$.

In Fig. 12 we show the empirical ratio of the differential cross sections as a proposal for the ratio of the gravitational form factors $A(\Delta t) / A\left(\Delta t_{\min }\right)$ with $\Delta t=\left(-\left(t-t_{\min }\right)\right)^{\frac{1}{2}}$ and $\Delta t_{\text {min }}=\sqrt{0.0075}$ versus $\Delta t^{2}$ in $\mathrm{GeV}^{2}$,

$$
\frac{A(\Delta t)}{A\left(\Delta t_{\min }\right)}=\left(\frac{F\left(s, t=t_{\min }, M_{V}, m_{N}\right)\left(-2 t_{\min }+8 m_{N}^{2}\right)}{F\left(s, t=-K^{2}, M_{V}, m_{N}\right)\left(2 K^{2}+8 m_{N}^{2}\right)}\right) \frac{\left(\frac{d \sigma}{d t}\right)}{\left(\frac{d \sigma}{d t}\right)_{\min }}
$$

The blue solid line is our holographic result (8.4), the red dashed line is the fitted lattice gluonic contribution from the recent simulations in [26], and the data are the ratio of the data from GlueX [15]. The empirical errors for the ratio

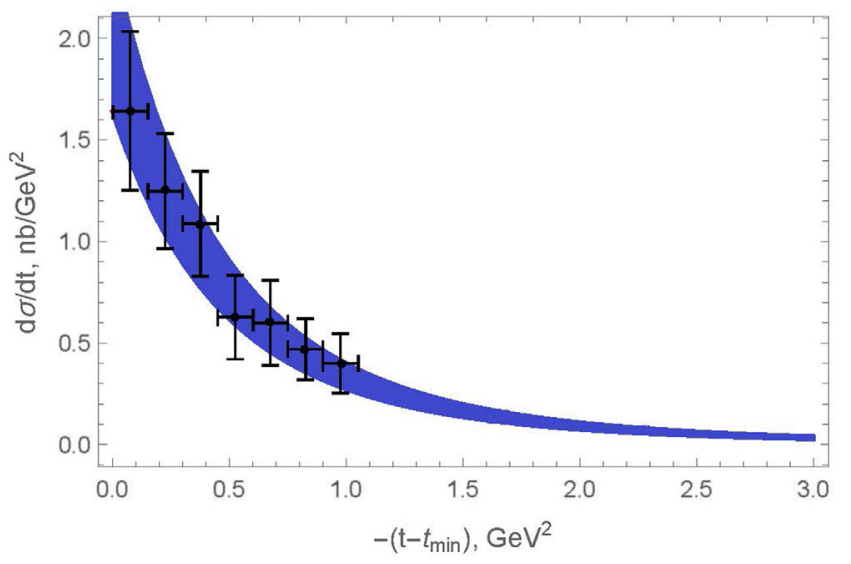

FIG. 10. Same differential cross section for $V=J / \Psi$ photoproduction with the normalization (8.9) changed by $\pm 10 \%$. 


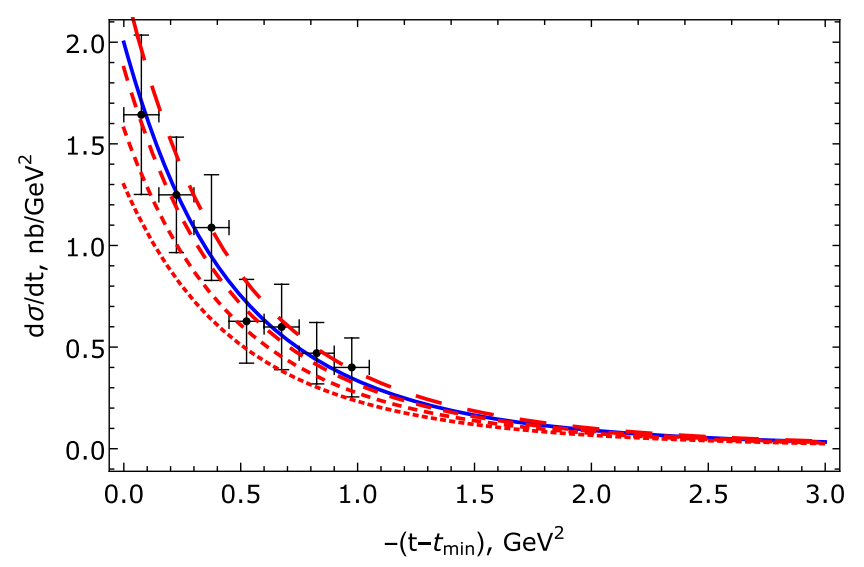

FIG. 11. Same differential cross section for $V=J / \Psi$ photoproduction but different photon energies: $E_{\gamma}=11 \mathrm{GeV}$ large red dashed line, $E_{\gamma}=10.72 \mathrm{GeV}$ solid blue curve, $E_{\gamma}=10.6 \mathrm{GeV}$ medium red dashed line, $E_{\gamma}=10.3 \mathrm{GeV}$ small red dashed line, and $E_{\gamma}=10 \mathrm{GeV}$ dotted red curve. The data are from GlueX [15].

have been added in quadrature. Equation (8.10) provides for a model independent extraction of the gravitational form factor, under two generic assumptions: (i) The Yukawa coupling of the dilaton to the bulk Dirac fermion vanishes in holography; and (ii) the tensor $2^{++}$glueball couplings map on the graviton couplings in bulk.

In Fig. 13 we show the differential cross section for $V=\Upsilon$ production close to threshold for different photon energies which is a prediction, for the same parameter set as the one used for $J / \Psi$ production. The photon energies are $E_{\gamma}=58.9 \mathrm{GeV}$ large red dashed line, $E_{\gamma}=58.6 \mathrm{GeV}$ medium red dashed line, $E_{\gamma}=58.45 \mathrm{GeV}$ solid blue curve, $E_{\gamma}=58.3 \mathrm{GeV}$ small red dashed line, and $E_{\gamma}=58 \mathrm{GeV}$ dotted red curve. We have used $m_{0}=M_{\Upsilon}=9.460 \mathrm{GeV}$,

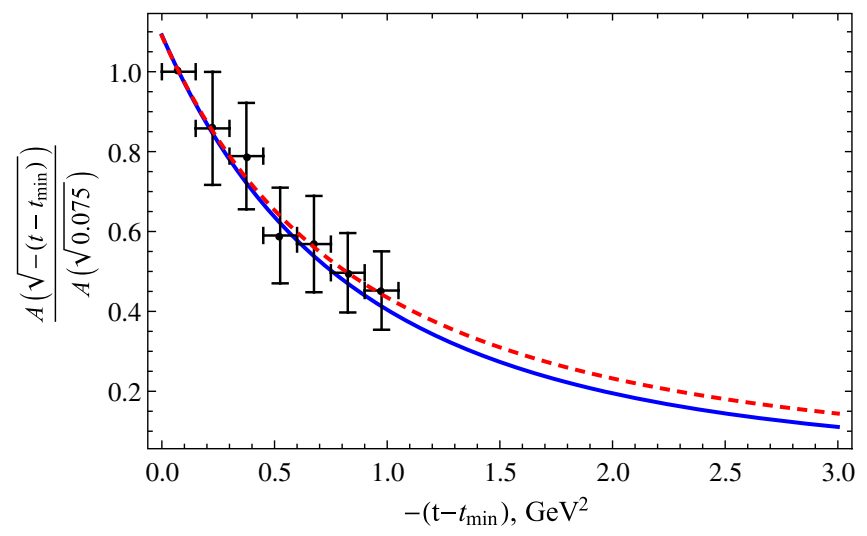

FIG. 12. The gravitational form factor $A\left(\sqrt{-\left(t-t_{\min }\right)}\right)$ [normalized by $A(\sqrt{0.075})]$ for $\tilde{\kappa}_{\rho}=0.350 \mathrm{GeV}, m_{N}=0.94 \mathrm{GeV}$, and $m_{J / \psi}=3.10 \mathrm{GeV}$. Blue solid line is our result, and dashed red line is from lattice QCD. We used the data from GlueX [15] with the errors added in quadrature.

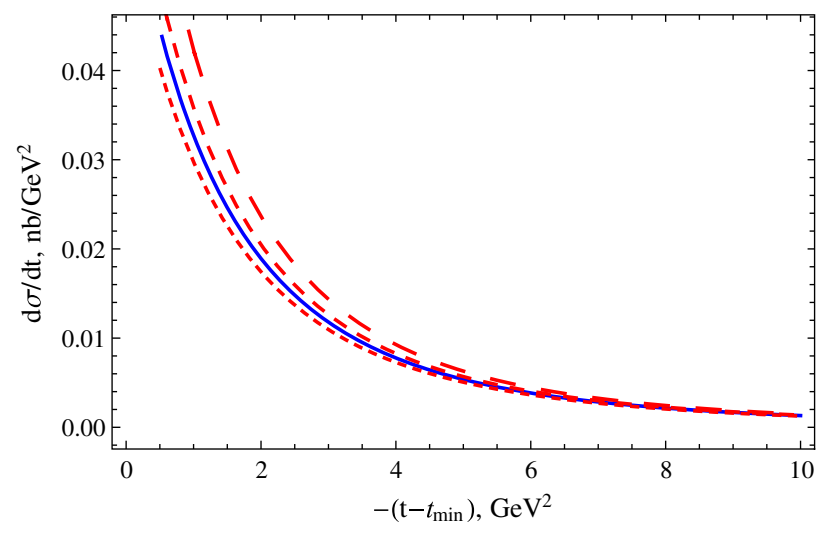

FIG. 13. Differential cross section for $V=\Upsilon$ and different photon energies: $E_{\gamma}=58.9 \mathrm{GeV}$ large red dashed line, $E_{\gamma}=$ $58.6 \mathrm{GeV}$ medium red dashed line, $E_{\gamma}=58.45 \mathrm{GeV}$ solid blue curve, $E_{\gamma}=58.3 \mathrm{GeV}$ small red dashed line, and $E_{\gamma}=58 \mathrm{GeV}$ dotted red curve.

$f_{0}=f_{\Upsilon}=0.714 \mathrm{GeV}$ and $\kappa_{V}$ as in (8.8) for the models with a soft wall. Note that in this case, $A(0)$ is fixed by the same ratios as in (8.9) with the numbers rescaled by the factor $\left(f_{J / \psi} m_{J / \psi} / f_{V} m_{V}\right)$ to correct for the $V=\Upsilon$ parameters.

In Fig. 14 (solid blue line), we show the total cross section for photoproduction of $V=J / \Psi$ versus the photon energy close to threshold. The total cross section follows by integrating the differential cross section in (8.4) using the dipole parametrization (10.2) with $k^{2} \rightarrow-t$. The comparison is to GlueX data [15] (black ones). All other holographic parameters are kept unchanged.

\section{REGGEIZED PHOTOPRODUCTION}

The differential cross section (8.4) grows rapidly as $s^{2}$ at large $s$ as expected from the exchange of a graviton as a tensor glueball exchange with spin- 2 . The physical cross

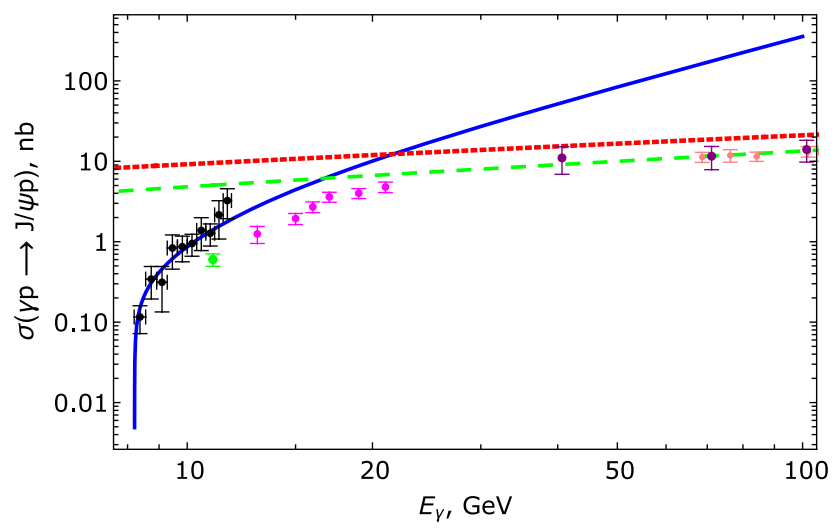

FIG. 14. The total cross section for $J / \Psi$ photoproduction with the same parameters as in Fig. 15, but zoomed in near threshold. The data points are from GlueX [15] (black), [30] (magneta), [31] (green), [32] (purple), and [33] (pink). 
section grows much slower due to the exchange of a Pomeron instead. The transmutation from a graviton to a Pomeron was originally discussed in [8]. With increasing $\sqrt{s}$, higher spin-j exchanges contribute leading to a reggeized amplitude with the emergence of a Pomeron. In this section and in supportive material given in the appendixes, we detail the spin- $j$ contribution to (8.4) and then resum these exchanges to extend the photoproduction results to all $\sqrt{s}$.

\section{A. Spin-j amplitude}

The spin-j exchange amplitude follows from the same considerations as the spin-2 exchange given earlier. Here we summarize the results for the soft-wall model with more details given in Appendix $\mathrm{C}$ together with the results for the hard-wall model. With this in mind, the spin-j glueball contribution to the TT-part of the photoproduction amplitude $\gamma p \rightarrow A p$ with an arbitrary virtual photon $A$, reads

$$
\begin{aligned}
i \mathcal{A}_{\gamma p \rightarrow A p}^{h}(j, s, t) \approx & (-i) \mathcal{V}_{h A A}^{\mu \nu(T T)}\left(j, q_{1}, q_{2}, k_{z}\right) \times\left(\frac{i}{2} \eta_{\mu \alpha} \eta_{\nu \beta}\right) \\
& \times(-i) \mathcal{V}_{h \bar{\Psi} \Psi}^{\alpha \beta(T T)}\left(j, p_{1}, p_{2}, k_{z}\right) .
\end{aligned}
$$

The explicit form of the tensor TT-vertices $\mathcal{V}^{T T}$ depend on the model used. For the soft-wall model, the normalized wave functions and bulk-to-bulk propagator are detailed in Appendix B. The result for the spin-j contribution to the vertices is

$$
\begin{aligned}
\mathcal{V}_{h A A}^{\mu \nu(T T)}\left(j, q_{1}, q_{2}, k_{z}\right) & =\frac{\sqrt{2 \kappa^{2}}}{2} \int d z \sqrt{g} e^{-\phi} z^{4+2(j-2)} K^{\mu \nu}\left(q, q^{\prime}, n, n^{\prime}, z\right) \times C(j) \times z^{\Delta(j)-j-2}, \\
\mathcal{V}_{h \bar{\Psi} \Psi}^{\alpha \beta(T T)}\left(j, p_{1}, p_{2}, k_{z}\right) & =-\frac{\sqrt{2 \kappa^{2}}}{2} \int d z \sqrt{g} e^{-\phi} z^{1+2(j-2)} \bar{\Psi}\left(p_{2}, z\right) \gamma^{\alpha} p^{\beta} \Psi\left(p_{1}, z\right) z^{-(j-2)} \mathcal{H}(j, K, z) \\
& =-\frac{\sqrt{2 \kappa^{2}}}{2} \int d z \sqrt{g} e^{-\phi} z^{1+2(j-2)}\left(\psi_{R}^{2}(z)+\psi_{L}^{2}(z)\right) z^{-(j-2)} \mathcal{H}(j, K, z) \times \bar{u}\left(p_{2}\right) \gamma^{\alpha} p^{\beta} u\left(p_{1}\right) \\
& =-\sqrt{2 \kappa^{2}} \times g_{5}^{2} A(j, K) \times \bar{u}\left(p_{2}\right) \gamma^{\alpha} p^{\beta} u\left(p_{1}\right),
\end{aligned}
$$

with the parameters

$$
\begin{aligned}
& C(j)=\tilde{\kappa}_{V}^{2 \Delta(j)-4} \times \frac{1}{\Delta(j)} \frac{2^{\Delta(j)-2} \Gamma\left(a_{K}+\frac{\Delta(j)}{2}\right)}{\Gamma(\Delta(j)-2)} \\
& \Delta(j)=2+\sqrt{2 \sqrt{\lambda}\left(j-j_{0}\right)} \text { and } a_{K}=\frac{a}{2}=\frac{K^{2}}{8 \tilde{\kappa}^{2}} \text { and } j_{0}=2-\frac{2}{\sqrt{\lambda}}
\end{aligned}
$$

For completeness, the analog vertex $\mathcal{V}_{h A A}^{\mu \nu(T T)}$ for the hard-wall model is

$$
\mathcal{V}_{h A A}^{\mu \nu(T T)}\left(j, q_{1}, q_{2}, k_{z}\right)=\frac{\sqrt{2 \kappa^{2}}}{2} \int d z \sqrt{g} e^{-\phi} z^{4+2(j-2)} K^{\mu \nu}\left(q, q^{\prime}, n, n^{\prime}, z\right) \frac{\frac{2^{1-\tilde{\Delta}(j)}}{\pi} \times K^{\tilde{\Delta}(j)} \times z^{\tilde{\Delta}(j)+2-(j-2)}}{\tilde{\Delta}(j)+2},
$$

Using (C26) in (9.2), we can write the spin-j form factor $A(j, K)$ of the proton as a bulk Dirac fermion in the soft-wall model as

$$
A(j, K)=\frac{1}{2 g_{5}^{2}} \frac{2^{2-\Delta(j)} \tilde{\kappa}_{N}^{j-2-\Delta(j)}}{\Gamma(\tilde{a}(j))} \int_{0}^{1} d x x^{\tilde{a}(j)-1}(1-x)^{-\tilde{b}(j)}\left(I_{z}^{R}(x)+I_{z}^{L}(x)\right),
$$

with

$$
\tilde{a}(j)=a_{K}+2-\frac{1}{2} \Delta(j) \quad \text { and } \quad \tilde{b}(j)=3-\Delta(j) .
$$

The integrals $\left(\xi=\tilde{\kappa}_{N}^{2} z^{2}\right)$

$$
I_{z}^{R / L}(x)=\int d z \sqrt{g} e^{-\phi} z^{1+2(j-2)} \psi_{R / L}^{2}(z) \xi^{\frac{-(j-2)}{2}} \xi^{2-\frac{\Delta(j)}{2}} \exp \left(-\frac{2 x \xi}{1-x}\right),
$$


are over the wave functions of the proton as a Dirac fermion in bulk in the soft-wall model. Specifically, we have

$$
\begin{aligned}
& \psi_{R}(z)=\frac{\tilde{n}_{R}}{\tilde{\kappa}_{N}^{\tau-2}} z^{\frac{5}{2} \xi^{\frac{\tau-2}{2}}} L_{0}^{(\tau-2)}(\xi), \\
& \psi_{L}(z)=\frac{\tilde{n}_{L}}{\tilde{\kappa}_{N}^{\tau-1}} z^{\frac{5}{2} \xi^{\frac{\tau-1}{2}}} L_{0}^{(\tau-1)}(\xi),
\end{aligned}
$$

with the twist parameter $\tau=7 / 2-1 / 2=3$. Here $L_{n}^{(\alpha)}(\xi)$ are the generalized Laguerre polynomials, and

$$
\tilde{n}_{R}=\tilde{n}_{L} \tilde{\kappa}_{N}^{-1} \sqrt{\tau-1}, \quad \tilde{n}_{L}=\tilde{\kappa}_{N}^{\tau} \sqrt{2 / \Gamma(\tau)} .
$$

Using the wave functions, the integrals in (9.7) can be carried out explicitly, with the results

$$
\begin{aligned}
& I_{z}^{R}(x)=\frac{1}{2} \times \tilde{\kappa}_{N}^{-2(j-2)} \times\left(\frac{\tilde{n}_{R}}{\tilde{\kappa}_{N}^{\tau-1}}\right)^{2} \times \int d \xi^{\frac{j-2}{2}+\tau-\frac{\Delta(j)}{2}}\left(L_{0}^{(\tau-2)}(\xi)\right)^{2} \exp \left(-\left(\frac{1+x}{1-x}\right) \xi\right) \\
& I_{z}^{L}(x)=\frac{1}{2} \times \tilde{\kappa}_{N}^{-2(j-2)} \times\left(\frac{\tilde{n}_{L}}{\tilde{\kappa}_{N}^{\tau}}\right)^{2} \times \int d \xi \xi^{\frac{j-2}{2}+\tau-\frac{\Delta(j)}{2}+1}\left(L_{0}^{(\tau-1)}(\xi)\right)^{2} \exp \left(-\left(\frac{1+x}{1-x}\right) \xi\right),
\end{aligned}
$$

where we used $\phi=e^{-\xi}$. Evaluating the integrals in (9.10) we obtain

$$
\begin{aligned}
& I_{z}^{R}(x)=\frac{1}{2} \times \tilde{\kappa}_{N}^{-2(j-2)} \times\left(\frac{\tilde{n}_{R}}{\tilde{\kappa}_{N}^{\tau-1}}\right)^{2} \times \Gamma\left(\frac{j-2}{2}+\tau-\frac{\Delta(j)}{2}+1\right) \times\left(\frac{1+x}{1-x}\right)^{-\frac{j-2}{2}-\tau+\frac{\Delta(j)}{2}-1} \\
& I_{z}^{L}(x)=\frac{1}{2} \times \tilde{\kappa}_{N}^{-2(j-2)} \times\left(\frac{\tilde{n}_{L}}{\tilde{\kappa}_{N}^{\tau}}\right)^{2} \times \Gamma\left(\frac{j-2}{2}+\tau-\frac{\Delta(j)}{2}+2\right) \times\left(\frac{1+x}{1-x}\right)^{-\frac{j-2}{2}-\tau+\frac{\Delta(j)}{2}-2} .
\end{aligned}
$$

Using (9.11) in (9.5), the spin-j glueball form factor of the proton becomes

$$
\begin{aligned}
A(j, K)= & \frac{1}{4 g_{5}^{2}} \frac{\tilde{\kappa}_{N}^{-(j-2)-\Delta(j)}}{\Gamma(\tilde{a}(j))} \int_{0}^{1} d x x^{\tilde{a}(j)-1}(1-x)^{-\tilde{b}(j)}\left(\left(\frac{\tilde{n}_{R}}{\tilde{\kappa}_{N}^{\tau-1}}\right)^{2} \times \Gamma(c(j))\left(\frac{1+x}{1-x}\right)^{-c(j)}\right. \\
& \left.+\left(\frac{\tilde{n}_{L}}{\tilde{\kappa}_{N}^{\tau}}\right)^{2} \times \Gamma(c(j)+1)\left(\frac{1+x}{1-x}\right)^{-(c(j)+1)}\right)
\end{aligned}
$$

with $\Delta(j)$ given in (9.3), $\tilde{a}(j), \tilde{b}(j)$ given in (9.6) and

$$
c(j)=(\tau+1)+\frac{j-2}{2}-\frac{\Delta(j)}{2} .
$$

$A(j, K)$ generalizes the gravitational form factor for all $j \geq 2$. Evaluating the integral in (9.12), we obtain (9.17). Inserting (9.17) in (9.2), (9.1) becomes

$$
\mathcal{A}_{\gamma p \rightarrow J / \Psi p}(j, s, t)=\mathcal{V}_{h A A}(j)\left(-\frac{1}{2} B_{1}^{\alpha \beta} \bar{u}\left(p_{2}\right) \gamma_{\alpha} p_{\beta} u\left(p_{1}\right)\right) \mathcal{V}_{h \bar{\Psi} \Psi}^{(T T)}(j)
$$

The spin-j vertices are

$$
\begin{aligned}
\mathcal{V}_{h \bar{\Psi} \Psi}^{(T T)}(j) & =-g_{5}^{2} A(j, K) \\
\mathcal{V}_{h A A}(j) & =\left(\frac{f_{n}}{m_{n}}\right) \times\left(\frac{\sqrt{2 \kappa^{2}}}{2} \int_{0}^{\infty} d z \sqrt{g} e^{-z^{2} \tilde{\kappa}_{V}^{2}} z^{4+2(j-2)} \times\left(2 \tilde{\kappa}^{2} z^{2}\right) L_{n}^{1}\left(z^{2} \tilde{\kappa}_{V}^{2}\right) \times C(j) \times z^{\Delta(j)-(j-2)}\right) \\
& \approx\left(\frac{f_{n}}{m_{n}}\right) \times\left(\frac{\sqrt{2 \kappa^{2}}}{2} \frac{L_{n}^{1}(0)}{\Delta(j) \tilde{\kappa}_{V}^{\Delta(j)+j-2}} \times C(j) \times\left(\int_{0}^{\infty} d \xi e^{-\xi^{2}} \xi^{\frac{\Delta(j)}{2}+\frac{j}{2}-1}\right)\right) \equiv\left(\frac{f_{V}}{M_{V}}\right) \mathbb{V}_{h A A}(j)
\end{aligned}
$$


with $B_{1}^{\alpha \beta}\left(q, q^{\prime}, n, n^{\prime}\right)$ defined in (4.22). The heavy mesons with $n=J / \Psi, \Upsilon$ are subsumed. Equation (9.14) shows how VMD extends to general spin-j exchange in holography, with $\mathbb{V}_{h A A}(j)$ reflecting on its coupling to the pair vector-mesonphoton in bulk.

\section{B. Reggeized amplitude}

After summing over all contributions from the spin-j glueballs, the photoproduction amplitude $\mathcal{A}_{\gamma p \rightarrow J / \Psi p}^{\text {tot }}(s, t)$ is

$$
\begin{aligned}
\mathcal{A}_{\gamma p \rightarrow J / \Psi p}^{\mathrm{tot}}(s, t) & =-\int_{\mathbb{C}} \frac{d j}{2 \pi i}\left(\frac{s^{j-2}+(-s)^{j-2}}{\sin \pi j}\right) \mathcal{A}_{\gamma p \rightarrow J / \Psi p}(j, s, t), \\
\mathcal{A}_{\gamma p \rightarrow J / \Psi p}(j, s, t) & =\frac{1}{2} \mathcal{V}_{h A A}(j) \times B_{1}^{\alpha \beta} \times \frac{2 \kappa^{2}}{g_{5}^{4}} \times g_{5}^{2} A(j, K) \times \bar{u}\left(p_{2}\right) \gamma_{\alpha} p_{\beta} u\left(p_{1}\right) .
\end{aligned}
$$

The contour $\mathbb{C}$ is at the rightmost of the branch point of $A(j, K)$. The spin-j glueball form factor $A(j, K)$ of the proton as a bulk Dirac fermion is given in (9.12) for the soft-wall model. The integrals can be carried explicitly, with the result

$$
\begin{aligned}
A(j, K)= & \frac{\tilde{\kappa}_{N}^{-(j-2)-\Delta(j)}}{4 g_{5}^{2}} \frac{\Gamma(c) \Gamma(1-\tilde{b}+c)}{\Gamma(1-\tilde{b}+c+\tilde{a})} \\
& \times\left(\left(\frac{\tilde{n}_{R}}{\tilde{\kappa}_{N}^{\tau-1}}\right)^{2}{ }_{2} F_{1}(\tilde{a}, c+1,1-\tilde{b}+c+\tilde{a},-1)+\left(\frac{\tilde{n}_{L}}{\tilde{\kappa}_{N}^{\tau}}\right)^{2} \frac{c(1-\tilde{b}+c)}{1-\tilde{b}+c+\tilde{a}^{2}} F_{1}(\tilde{a}+1, c+1,2-\tilde{b}+c+\tilde{a},-1)\right) .
\end{aligned}
$$

The parameters are fixed in (9.3) as

$$
\begin{aligned}
& 1-\tilde{b}+c=(\tau-1)+\frac{j-2}{2}+\frac{\Delta(j)}{2}, \\
& 1-\tilde{b}+c+\tilde{a}=(\tau+1)+\frac{j-2}{2}+a_{K} .
\end{aligned}
$$

Note that at $j=2,(9.17)$ is exactly equal to the spin-2 gravitational form factor (5.5) (times $1 / \tilde{\kappa}_{V}^{4}$ to compensate for the new normalization we used for the higher spin case).

From (9.19)-(9.17), we determine the single Pomeron amplitude (total amplitude) in momentum space, after wrapping the $\mathrm{j}$-plane contour $\mathbb{C}$ to the left,

$$
\mathcal{A}_{\gamma p \rightarrow J / \Psi p}^{\mathrm{tot}}(s, t)=-s^{j_{0}-2} \int_{-\infty}^{j_{0}} \frac{d j}{\pi}\left(\frac{1+e^{-i \pi}}{\sin \pi j}\right) s^{j-j_{0}} \operatorname{Im}\left[\mathcal{A}_{\gamma p \rightarrow J / \Psi p}(j, s, t)\right] .
$$

The imaginary part follows from the discontinuity of the $\Gamma$-function

$$
\begin{aligned}
& \operatorname{Im}\left[\mathcal{A}_{\gamma p \rightarrow J / \Psi p}(j, s, t)\right] \approx \frac{\tilde{\kappa}_{N}^{-(j-2)-\Delta(j)}}{\tilde{\kappa}_{N}^{4-\Delta(j)+j-2}} \times\left(\frac{\tilde{\kappa}_{N}}{\tilde{\kappa}_{V}}\right)^{4-\Delta(j)+j-2} \times \frac{\sqrt{2 \kappa^{2}}}{g_{5}^{4}} \\
& \times\left.\left(\frac{1}{2} \tilde{\kappa}_{V}^{4-\Delta(j)+j-2} \Gamma(\Delta(j)-2) \mathcal{V}_{h A A}(j) \times B_{1}^{\alpha \beta} \times \tilde{\kappa}_{N}^{j-2+\Delta(j)} g_{5}^{2} A(j, K) \bar{u}\left(p_{2}\right) \gamma_{\alpha} p_{\beta} u\left(p_{1}\right)\right)\right|_{j \rightarrow j_{0}, \Delta(j) \rightarrow 2} \\
& \times \operatorname{Im}\left[\frac{1}{\Gamma(\tilde{\Delta}(j))}\right]
\end{aligned}
$$

with the complex argument

$$
\tilde{\Delta}(j)=\Delta(j)-2=i \sqrt{2 \sqrt{\lambda}\left(j_{0}-j\right)} \equiv i y
$$

and $j_{0}=2-2 / \sqrt{\lambda}$. For $y \rightarrow 0$, we may approximate $1 / \Gamma(i y) \approx i y e^{i \gamma y}$, with the Euler-Mascheroni constant $\gamma=0.55772 \ldots$ The single Pomeron amplitude (total amplitude) in momentum space (9.19) can now be cast in block form 


$$
\mathcal{A}_{\gamma p \rightarrow J / \Psi p}^{\mathrm{tot}}(s, t)=I_{j}\left(j_{0}, s\right) \times G_{5}\left(j_{0}, s, t\right)
$$

with

$$
\begin{aligned}
I_{j}\left(j_{0}, s\right)= & -\tilde{s}^{j_{0}} \int_{-\infty}^{j_{0}} \frac{d j}{\pi}\left(\frac{1+e^{-i \pi}}{\sin \pi j}\right) \tilde{s}^{j-j_{0}} \sin \left[\tilde{\xi} \sqrt{2 \sqrt{\lambda}\left(j_{0}-j\right)}\right], \\
G_{5}\left(j_{0}, s, t\right)= & \left(\frac{\tilde{\kappa}_{N}}{\tilde{\kappa}_{V}}\right)^{4-\Delta(j)+j-2} \times \frac{1}{s^{2}}\left(\frac{1}{2} \tilde{\kappa}_{V}^{4-\Delta(j)+j-2} \Gamma(\Delta(j)-2) \mathcal{V}_{h A A}(j) \times B_{1}^{\alpha \beta} \times \frac{\sqrt{2 \kappa^{2}}}{g_{5}^{4}}\right. \\
& \left.\times \tilde{\kappa}_{N}^{j-2+\Delta(j)} g_{5}^{2} A(j, K) \bar{u}\left(p_{2}\right) \gamma_{\alpha} p_{\beta} u\left(p_{1}\right)\right)\left.\right|_{j \rightarrow j_{0}, \Delta(j) \rightarrow 2} .
\end{aligned}
$$

We have set $\tilde{s} \equiv s / \tilde{\kappa}_{N}^{2}$, and defined $\tilde{\xi} \equiv \gamma+\pi / 2$. We note that the apparent pole in the Gamma function at the Pomeron intercept cancels out in the combination $\Gamma\left(\Delta\left(j_{0}\right)-2\right) \mathcal{V}_{h A A}\left(j_{0}\right)$.

In the block form (9.22), the spin-j integral $I_{j}\left(j_{0}, s\right)$ is similar to the spin-j integral in [8] [see Eq. (4.19)], with the identifications $\mathcal{K}\left(s, b^{\perp}, z, z^{\prime}\right) \leftrightarrow \mathcal{A}_{\gamma p \rightarrow J / \Psi p}^{\text {tot }}(s, t),\left(z z^{\prime} / R^{4}\right) G_{3}\left(j_{0}, v\right) \leftrightarrow G_{5}\left(j_{0}, s, t\right), \xi(v) \leftrightarrow \tilde{\xi}$, and $\hat{s} \leftrightarrow \tilde{s}$. We then follow [8] to evaluate the spin-j integral by closing the j-contour appropriately. In the high-energy limit $\sqrt{\lambda} / \tilde{\tau} \rightarrow 0(\tilde{\tau} \equiv \log \tilde{s})$, the single Pomeron contribution to the photoproduction amplitude is

$$
\mathcal{A}_{\gamma p \rightarrow J / \Psi p}^{\mathrm{tot}}(s, t) \simeq e^{j_{0} \tilde{\tau}}[(\sqrt{\lambda} / \pi)+i](\sqrt{\lambda} / 2 \pi)^{1 / 2} \frac{e^{-\sqrt{\lambda} \tilde{\xi}^{2} / 2 \tilde{\tau}}}{\tilde{\tau}^{3 / 2}}\left(1+\mathcal{O}\left(\frac{\sqrt{\lambda}}{\tilde{\tau}}\right)\right) \times G_{5}\left(j_{0}, s, t\right) .
$$

As expected, the amplitude develops both a real and imaginary part with a $\rho$-ratio about constant

$$
\rho=\frac{\operatorname{Re}\left[\mathcal{A}_{\gamma p \rightarrow J / \Psi p}^{\mathrm{tot}}(s, t=0)\right]}{\operatorname{Im}\left[\mathcal{A}_{\gamma p \rightarrow J / \Psi p}^{\mathrm{tot}}(s, t=0)\right]} \simeq \frac{\sqrt{\lambda}}{\pi} .
$$

The single Pomeron contribution to the total differential cross section is

$$
\begin{aligned}
\left(\frac{d \sigma}{d t}\right)_{\mathrm{tot}} & =\frac{e^{2}}{16 \pi\left(s-m_{N}^{2}\right)^{2}} \frac{1}{2} \sum_{\mathrm{pol}} \frac{1}{2} \sum_{\mathrm{spin}}\left|\mathcal{A}_{\gamma p \rightarrow J / \Psi p}^{\mathrm{tot}}(s, t)\right|^{2} \\
& \simeq \frac{e^{2}}{16 \pi\left(s-m_{N}^{2}\right)^{2}} \times\left(e^{2 j_{0} \tilde{\tau}}\left[\left(\lambda / \pi^{2}\right)+1\right](\sqrt{\lambda} / 2 \pi) \tilde{\xi}^{2} \frac{e^{-\sqrt{\lambda \xi^{2}} / \tilde{\tau}}}{\tilde{\tau}^{3}}\right) \times \frac{1}{2} \sum_{\mathrm{pol}} \frac{1}{2} \sum_{\mathrm{spin}}\left|G_{5}\left(j_{0}, s, t\right)\right|^{2}
\end{aligned}
$$

with the polarization-spin average

$$
\begin{aligned}
\sum_{\text {pol,spin }}\left|G_{5}\left(j_{0}, s, t\right)\right|^{2}= & \left.\left(\frac{\tilde{\kappa}_{N}}{\tilde{\kappa}_{V}}\right)^{2(4-\Delta(j)+j-2)}\left(\frac{f_{V}}{M_{V}}\right)^{2}\left(\frac{2 \kappa^{2}}{g_{5}^{8}} \tilde{\kappa}_{V}^{2(4-\Delta(j)+j-2)} \Gamma^{2}(\Delta(j)-2) \mathbb{V}_{h A A}^{2}(j) \times \tilde{\kappa}_{N}^{2(j-2+\Delta(j))} \frac{g_{5}^{4} A^{2}(j, K)}{m_{N}^{2}}\right)\right|_{j \rightarrow j_{0}, \Delta(j) \rightarrow 2} \\
& \times \frac{F\left(s, t=-K^{2}, M_{V}, m_{N}\right)}{s^{4}} \times\left(2 K^{2}+8 m_{N}^{2}\right)
\end{aligned}
$$

and $j_{0}=2-2 / \sqrt{\lambda}$. Note that the resummed spin-j contribution to the gravitational form factor is now fixed by the Pomeron exchange with the form factor $A\left(K, j_{0}\right)$ at large $\sqrt{s}$. Remarkably, the emerging Pomeron exchange in the soft-wall model in (9.26) which is a new result, bears much in common with the original conformal Pomeron kernel in [8].

The differential cross section rises with twice the conformal Pomeron intercept or $2 \times(1-2 / \sqrt{\lambda})$, and asymptotes

$$
\left(\frac{d \sigma}{d t}\right)_{\text {tot }} \sim s^{2-\frac{4}{\sqrt{\lambda}}} \times\left(1+\frac{\pi^{2}}{\lambda}\right)\left(\left(\frac{\sqrt{\lambda}}{\log \tilde{s}}\right)^{3}+\mathcal{O}\left(\left(\frac{\sqrt{\lambda}}{\log \tilde{s}}\right)^{4}\right)\right)
$$

in the high-energy limit with $\log \tilde{s}=\log \left(s / \tilde{\kappa}_{V}^{2}\right) \gg \sqrt{\lambda}$. Using the optical theorem one can determine the total cross section $\sigma_{V}(s)$ for $\gamma p \rightarrow V p$ with $V=J / \Psi, \Upsilon$ to be 


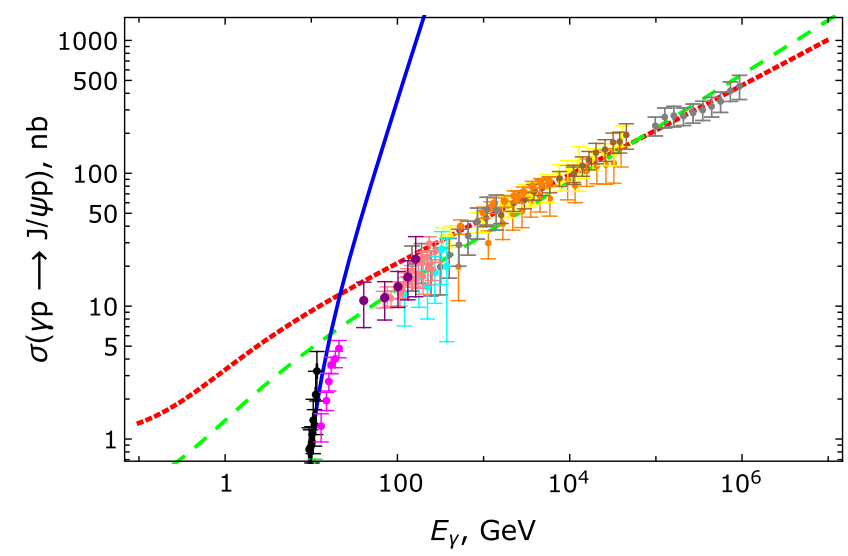

FIG. 15. Total cross section for photoproduction of charmonium with $V=J / \Psi$, from close to threshold to very high energy. The solid (blue) curve is the low-energy regime compared to the data from GlueX [15] (black). The red (tiny dashed line) is the high-energy regime. The green line (medium dashed line) is found after fixing a normalization constant with one high-energy data point but with the same high-energy 't Hooft coupling constant $\lambda=11.243$ as the red (tiny dashed) one. The data points are from [30] (magneta), [31] (green), [32] (purple), [34] (orange), [33] (pink), [35] (yellow), [36] (brown), [37] (orange), and [38] (gray).

$$
\sigma_{V}(s)=\left(\frac{16 \pi}{1+\rho^{2}}\left(\frac{d \sigma}{d t}\right)_{\mathrm{tot}}\right)_{t=0}^{\frac{1}{2}}
$$

with the Pomeron rise $\sigma_{V}(s) \sim s^{1-2 / \sqrt{\lambda}}$ at large $\sqrt{s}$ [8]. Recall that close to threshold, the t-exchange is kinematically bounded as shown in Fig. 1, and the total cross section follows from the differential cross section (8.4) by integration using (2.7).

In Fig. 15, we show the total cross section for photoproduction of charmonium with $V=J / \Psi$ from threshold to very high energy. The same soft-wall parameters (8.8) and the same fitting condition on $A(0)$ as in (8.9) are used in the threshold region for the solid blue curve. In this region, the parameter set is insensitive to the expansion of the vector meson wave function $L_{n}^{1}(z)$ near the holographic boundary. At very high energy, we used the parameter set (8.8) for the D9 model and adjusted $A\left(0, j_{0}\right)$ to

$$
\left(\frac{\tilde{\kappa}_{N}}{\tilde{\kappa}_{V}}\right)^{j_{0}} \times \frac{10 N_{f} A\left(0, j_{0}\right)}{\sqrt{\lambda}\left(10 \tilde{\kappa}_{V}\right)^{4}}=3.631 \mathrm{GeV}^{-4} \quad(\mathrm{D} 9 \text { model })
$$

with $\lambda=11.243$. The fit value (9.30) is sensitive to the expansion of $L_{n}^{1}(z)$ near the holographic boundary. The value of the coupling $\lambda$ is not. Similar fits are found for the other two holographic models. The solid (blue) curve is the low-energy regime. The data are from GlueX [15] (black). The red (tiny dashed line) is the high-energy regime. The green line (medium dashed) is found after fixing a

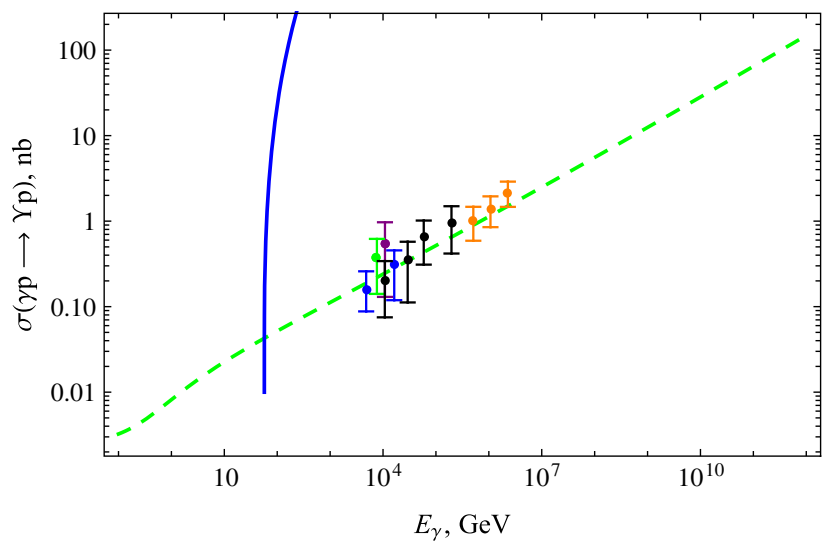

FIG. 16. Total cross section for photoproduction of upsilonium with $V=\Upsilon$ from close to threshold to the very high-energy regime. The solid (blue) curve is the low-energy regime (near threshold). The green line (medium dashed) is found after fixing a normalization constant with one high-energy data point but with the same 't Hooft coupling constant $\lambda=11.243$ as $J / \psi$. The data points are from [39] (green), [40] (purple), [41] (blue), [42] (orange), and [43] (black).

normalization constant with one high-energy data point but with the same high-energy 't Hooft coupling constant $\lambda=$ 11.243 as the red (tiny dashed) one. In Fig. 14, we zoomed in the total cross section for $J / \Psi$ photoproduction near the threshold with the same parametrs as in Fig. 15, and compared to data from GlueX [15] in this regime.

In Fig. 16, we show the total cross section for $V=\Upsilon$ photoproduction from close to threshold to very highenergy regime, with the same parameter set. The solid (blue) curve is the low-energy regime. The green line (medium dashed) is found after fixing a normalization constant with one high-energy data point but with the same 't Hooft coupling constant $\lambda=11.243$ as $J / \psi$. In Fig. 17, we show the total cross section for $\gamma$ photoproduction zoomed in close to the threshold with the same parameters as in Fig. 16.

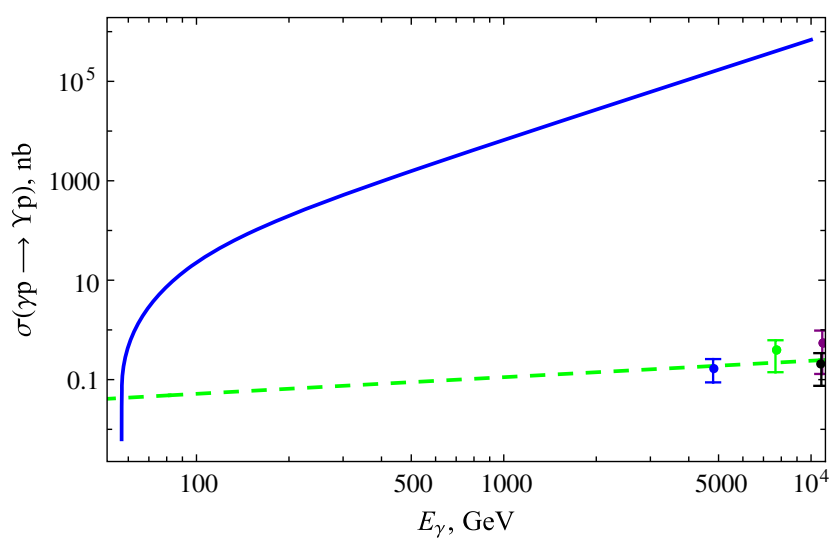

FIG. 17. Total cross section for $\Upsilon$ photoproduction with the same parameters as in Fig. 16 but zoomed in near the threshold. 


\section{GENERALIZED PARTON DISTRIBUTION OF GLUONS INSIDE THE PROTON}

The generalized parton distribution can be viewed as the amplitude for removing a parton with momentum fraction-x and then reinserting it, while the nucleon is receiving a momentum kick $\vec{K}$ all the while traveling on the light cone. It is related to the form factor of the energy-momentum tensor by a sum rule as we now detail. The fact that the gluon GPD can be picked in the diffractive photoproduction of heavy mesons is not surprising. Indeed, as we noted earlier, the Witten diagram for the holographic photoproduction amplitude is related to the amplitude for the inverse deeply virtual Compton scattering amplitude through VMD.

\section{A. Gluon GPD: $j=2$}

The tensor coupling of the glueball to the nucleon as a Dirac fermion is through its gravitational invariant form factors (5.3). For $j=2$ the exchange is dominated by the graviton at threshold, with the contribution $(\tau=3$ and $\left.a_{K}=K^{2} / 8 \tilde{\kappa}_{N}^{2}\right)$

$$
\begin{aligned}
A(K) & =A(0) \times \Gamma\left(a_{K}+2\right) \times g_{5}^{2} \tilde{\kappa}_{N}^{4} A(j=2, K) \\
& =A(0) \int_{0}^{1} d x \frac{a_{K}\left(a_{K}+1\right)}{x^{\alpha_{G}(t)}}\left(\frac{1-x}{1+x}\right)^{\tau}
\end{aligned}
$$

with the graviton Regge trajectory $\alpha_{G}(t)=1+t / m_{0}^{2}$ and $-t=K^{2} \ll s$. Here $m_{0}$ is fixed by the $2^{++}$glueball mass in (4.11). For a spin-2 and twist-2 exchange, the $A(K)$ form factor obeys the sum rule [27] (see also Eq. (3.154) in [44], and reference therein)

$$
A(K) \equiv \int_{0}^{1} d x x^{j-1} g(x, K)
$$

with $x g(x, K)$ the gluon GPD, at the renormalization scale set by the nucleon mass.

The representation (10.1) suggests that $0 \leq x \leq 1$ maybe interpreted as the $\mathrm{x}$-momentum fraction of the gluons in the proton probed by the graviton. At small-x, the exchange is dominated by the graviton Regge trajectory which is manifest in the integral representation (10.1)

$$
x g_{<}(x, K) \sim A(0) \frac{a_{K}\left(a_{K}+1\right)}{x^{\alpha_{G}(t)}}\left(\frac{1-x}{1+x}\right)^{\tau} .
$$

For zero skewness $(\xi=0)$, the momentum transfer is purely transverse and the spatial and transverse Fourier transform of (10.3) samples the distribution of an x-parton at a given transverse spatial distance in the light cone,

$$
x g_{<}\left(x, b_{\perp}\right)=\int \frac{d K_{\perp}}{(2 \pi)^{2}} e^{-i K_{\perp} \cdot b_{\perp}} x g_{<}\left(x, K_{\perp}\right)
$$

with

$$
\begin{aligned}
b_{\perp}^{2} x g_{<}\left(x, b_{\perp}\right) \sim & A(0) \frac{2 \tilde{\kappa}_{N}^{2} b_{\perp}^{2}}{\pi x}\left(\frac{1-x}{1+x}\right)^{\tau} \frac{e^{\frac{2 \tilde{\kappa}_{N}^{2} b_{\perp}^{2}}{\ln x}}}{\ln ^{5} x} \\
& \times\left(-4 \tilde{\kappa}_{N}^{4} b_{\perp}^{4}+\ln x\left(-8 \tilde{\kappa}_{N}^{2} b_{\perp}^{2}\right.\right. \\
& \left.\left.+\ln x\left(-2+2 \tilde{\kappa}_{N}^{2} b_{\perp}^{2}+\ln x\right)\right)\right) .
\end{aligned}
$$

Equation (10.5) is seen to spread or diffuse (Gribov diffusion) in the transverse plane over a length scale fixed by $l_{\perp} \sim(2 \ln (1 / x))^{\frac{1}{2}} / \tilde{\kappa}_{N}$, with

$b_{\perp}^{2} x g_{<}\left(x, b_{\perp}\right) \sim A(0) \frac{8\left(\tilde{\kappa}_{N} b_{\perp}\right)^{6}}{\pi x}\left(\frac{1-x}{1+x}\right)^{\tau} \frac{e^{-\frac{2 \tilde{N}^{2} b_{\perp}^{2}}{\ln \frac{1}{x}}}}{\ln ^{5} \frac{1}{x}}$

which is enhanced at low-x as $1 /\left(x \ln ^{5} \frac{1}{x}\right)$. The diffusion ceases to be semipositive for $b_{\perp}<l_{\perp}$ or $K_{\perp}>1 / l_{\perp}$. In Fig. 18 we show the behavior of the transverse gluon density (10.6) as probed by the graviton at small-x and small $K_{\perp}$ or large $b_{\perp}$. The central hole in Fig. 18(b) occurs at small $b_{\perp}<l_{\perp}$ and falls outside the range of the diffusive approximation in (10.6).

To probe large- $\mathrm{x}$ and small $b_{\perp}$ through (10.1), it is best to remove the large $\mathrm{K}$-factors in the integrand through two integrations by parts without modifying the sum rule for $A(K)$. The result is

$$
x g_{>}(x, K) \sim A(0) x^{a_{K}+1}\left(\left(\frac{1-x}{1+x}\right)^{\tau}\right)^{\prime \prime}
$$

with the primes referring to $\mathrm{x}$-derivatives. The corresponding transverse density at large-x is semipositive throughout, and reads

$b_{\perp}^{2} x g_{>}\left(x, b_{\perp}\right) \sim A(0) x\left(\left(\frac{1-x}{1+x}\right)^{\tau}\right)^{\prime \prime} \frac{2\left(\tilde{\kappa} b_{\perp}\right)^{2}}{\pi} \frac{e^{-\frac{2 \tilde{R}_{N}^{2} b_{\perp}^{2}}{\ln \frac{1}{x}}}}{\ln \frac{1}{x}}$

In Fig. 19(a) we show the large-x behavior of the gluon GPD (10.8) as probed by the graviton, as a function of parton-x and the rescaled transverse size $\tilde{\kappa}_{N} b_{\perp}$ for $\tau=3$. The GPD distribution for large-x and fixed $x=0.5$ in the transverse plane is shown in Fig. 19(b). For comparison, one can look at the GPD of valence quarks in the proton extracted from holographic QCD models in [45].

\section{B. Gluon GPD: $j=j_{0}$}

Higher spin-j exchanges once resummed yield Pomeron exchange at higher energies. The emerging Pomeron form factor follows from (9.12) for $j=j_{0}$ in the form 


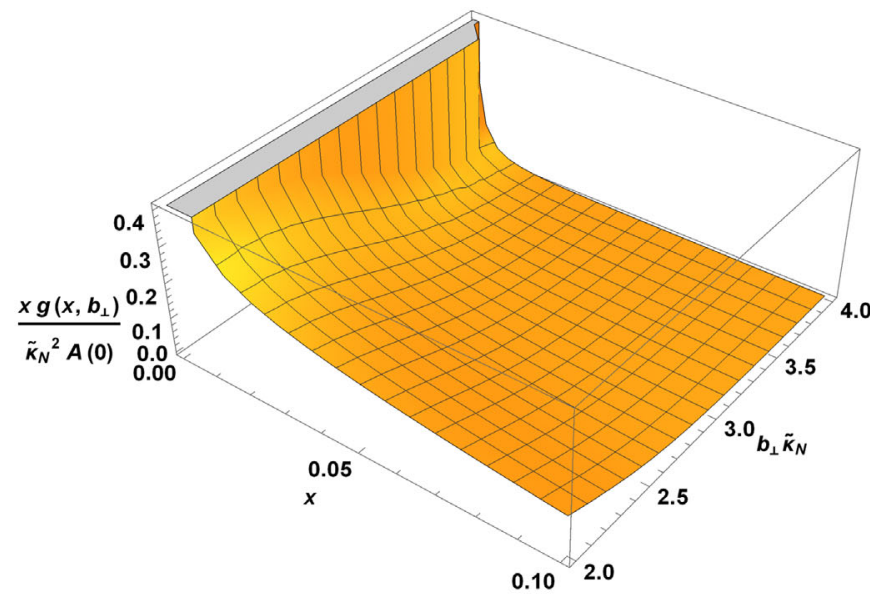

(a) The small-x gluon distribution $x g_{<}\left(x, b_{\perp}\right)$ inside the proton (X.100) as probed by the graviton.

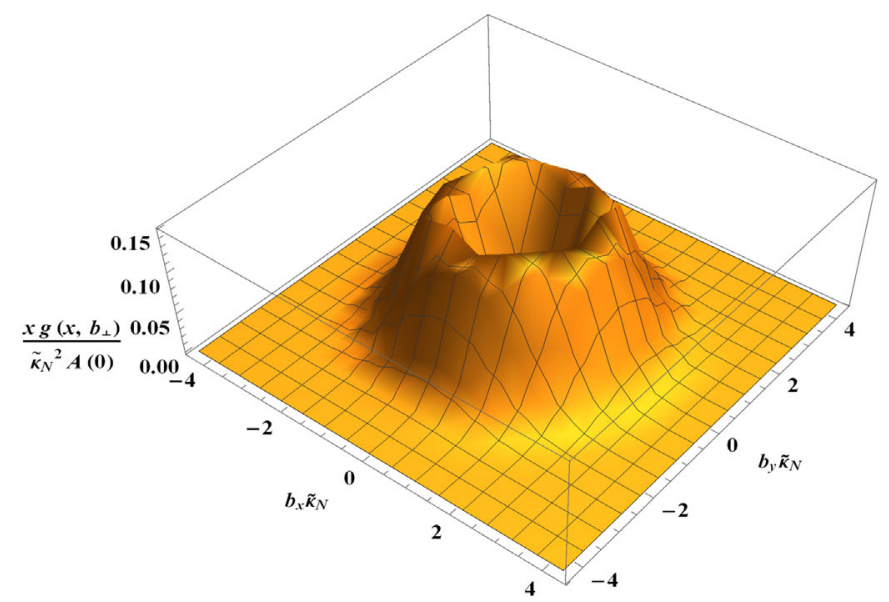

(b) The small-x gluon $x g_{<}\left(x, b_{\perp}\right)$ distribution inside the proton (X.100) as probed by the graviton with $\tau=3, x=0.1$ and $b_{\perp}=\left(b_{x}^{2}+b_{y}^{2}\right)^{\frac{1}{2}}$.

FIG. 18. Small-x gluon GPD as probed by graviton exchange in photoproduction of a heavy meson close to threshold.

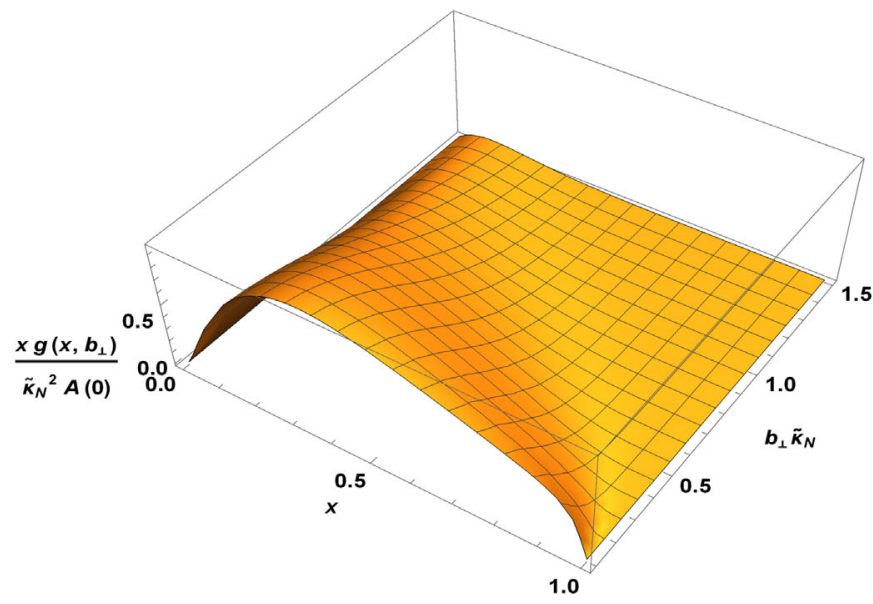

(a) The large-x gluon distribution $x g_{>}\left(x, b_{\perp}\right)$ inside the proton (X.102) as probed by the graviton with $\tau=3$.

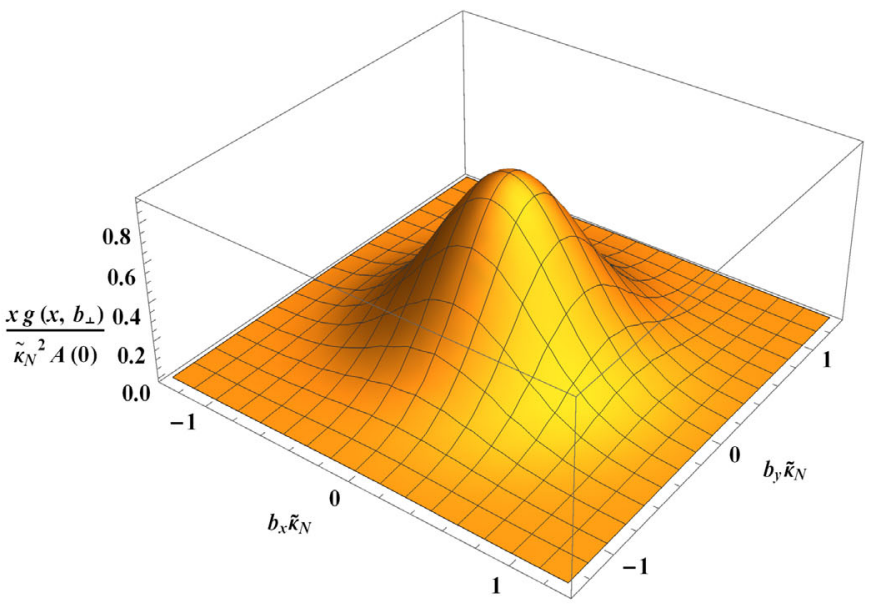

(b) The large-x gluon distribution $x g_{>}\left(x, b_{\perp}\right)$ inside the proton (X.102) as probed by the graviton with $\tau=3, x=0.5$ and

$$
b_{\perp}=\left(b_{x}^{2}+b_{y}^{2}\right)^{\frac{1}{2}} .
$$

FIG. 19. Large-x gluon GPD as probed by graviton exchange in photoproduction of a heavy meson close to threshold.

$$
\begin{aligned}
A_{P}(K) & =A\left(j_{0}, 0\right) \Gamma\left(a_{K}+\Delta\left(j_{0}\right) / 2\right) \times g_{5}^{2} \tilde{\kappa}_{N}^{j_{0}-2+\Delta\left(j_{0}\right)} A\left(j_{0}, K\right) \\
& =A\left(j_{0}, 0\right) \frac{\Gamma(\tau-1 / \sqrt{\lambda})}{\Gamma(\tau)} \int_{0}^{1} d x x^{j_{0}-1} \frac{1}{x^{\alpha_{P}(t)}}\left(\frac{1-x}{1+x}\right)^{\tau-\frac{1}{\sqrt{\lambda}}} \frac{1}{1-x^{2}}\left((\tau-1)(1+x)+\left(\tau-\frac{1}{\sqrt{\lambda}}\right)(1-x)\right)
\end{aligned}
$$

with the Pomeron trajectory $\alpha_{P}(t)=1-2 / \sqrt{\lambda}+t / m_{0}^{2}$, and with $m_{0}$ fixed by the $2^{++}$glueball mass in (4.11). By analogy with the $j=2$ exchange, we suggest that the gluon content of the proton as probed by the Pomeron for small $K$ is concentrated at small-x, and follows from the dominant Pomeron exchange which is manifest in (10.9) as

$$
x g(x, K) \sim A\left(j_{0}, 0\right) \frac{\Gamma(\tau-1 / \sqrt{\lambda})}{\Gamma(\tau)} \frac{1}{x^{\alpha_{P}(t)}}\left(\frac{1-x}{1+x}\right)^{\tau-\frac{1}{\sqrt{\lambda}}} \frac{1}{1-x^{2}}\left((\tau-1)(1+x)+\left(\tau-\frac{1}{\sqrt{\lambda}}\right)(1-x)\right) .
$$

The corresponding transverse gluon density is 


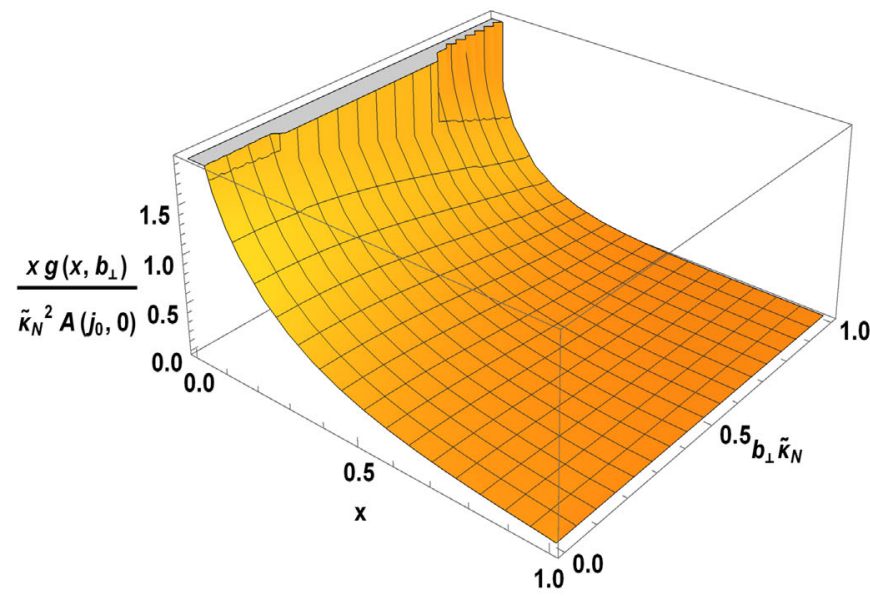

(a) The small-x gluon density inside the proton (X.105) as probed by the Pomeron with $\lambda=11.243$, and $\tau=3$.

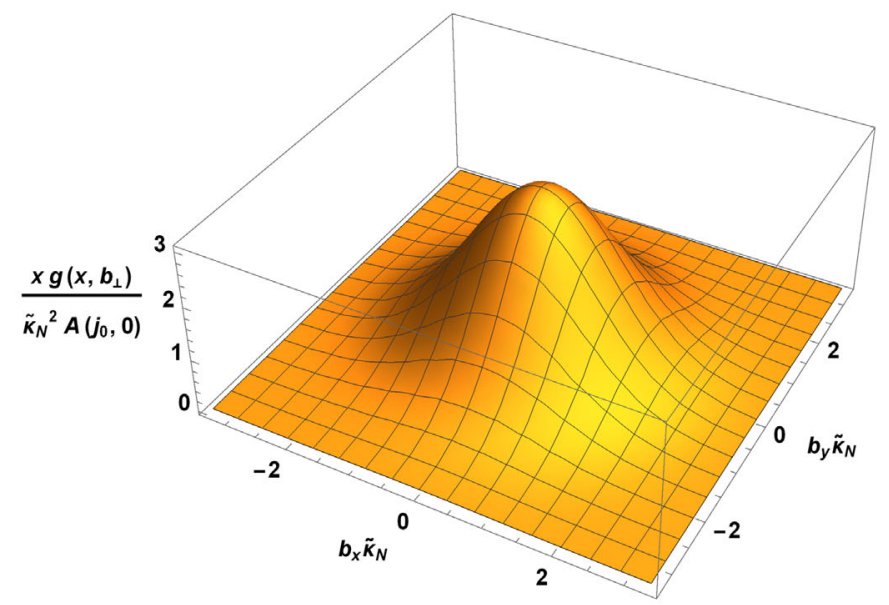

(b) The small-x gluon distribution inside the proton (X.105) as probed by the Pomeron with $\lambda=11.243, \tau=3, x=0.01$ and $b_{\perp}=\left(b_{x}^{2}+b_{y}^{2}\right)^{\frac{1}{2}}$.

FIG. 20. Gluon GPD as probed by Pomeron exchange in photoproduction of a heavy meson at high energy.

$$
b_{\perp}^{2} x g\left(x, b_{\perp}\right) \sim A\left(j_{0}, 0\right) \frac{\Gamma(\tau-1 / \sqrt{\lambda})}{\Gamma(\tau)}\left(\frac{1-x}{1+x}\right)^{\tau-\frac{1}{\sqrt{\lambda}}} \frac{1}{1-x^{2}}\left((\tau-1)(1+x)+\left(\tau-\frac{1}{\sqrt{\lambda}}\right)(1-x)\right) \frac{2\left(\tilde{\kappa} b_{\perp}\right)^{2}}{\pi x^{1-\frac{2}{\sqrt{\lambda}}}} \frac{e^{-\frac{2 \kappa_{N}^{2} b_{\perp}^{2}}{\ln \frac{1}{\lambda}}}}{\ln \frac{1}{x}}
$$

In Fig. 20(a) we show the behavior of the transverse gluon density probed by the Pomeron in (10.11), for $\lambda=$ 11.243, $\tau=3$. The same density is shown in Fig. 20(b) for fixed $x=0.01$. We note that the low-x contribution probed by the Pomeron at high energy or equivalently large rapidity $\chi=\ln \left(s / s_{\text {tr }}\right) \gg 1$ far from threshold, is substantially larger than the one probed by the graviton close to threshold at small rapidity $\chi=\ln \left(s / s_{\text {tr }}\right) \sim 1$. Also, we note that at high energy, the transverse gluon density probed by the Pomeron is diffusivelike throughout.

\section{Skewed Gluon GPD}

The gluonic skewed GPD for the energy-momentum tensor with finite skewness $\xi=K_{z} / 2 \sqrt{m_{N}^{2}+K^{2} / 4}$ are related to the invariant form factors in (5.3) through (see Eqs. (3.127) and (3.151) in [44], and references therein)

$$
\begin{aligned}
& \int_{0}^{1} d x H^{g}(x, \xi, K)=A(K)+\xi^{2} D(K) \rightarrow\left(1-4 \xi^{2}\right) A(K), \\
& \int_{0}^{1} d x E^{g}(x, \xi, K)=B(K)-\xi^{2} D(K) \rightarrow 4 \xi^{2} A(K)
\end{aligned}
$$

with the rightmost results following from our holographic results for the invariant form factors, $B(K)=0$ and $D(K)=4 C(K)=-4 A(K)$. In terms of (10.6)-(10.8) (graviton) or (10.10) (Pomeron), we have for the skewed gluonic distributions

$$
\begin{aligned}
H^{g}(x, \xi, K) & =\left(1-4 \xi^{2}\right) x g(x, K), \\
E^{g}(x, \xi, K) & =4 \xi^{2} x g(x, K),
\end{aligned}
$$

which amounts to the gluonic contribution to Ji's sum rule [46] as

$J^{\text {gluon }}(0)=\frac{1}{2} \int_{0}^{1} d x\left(H^{g}(x, \xi, 0)+E^{g}(x, \xi, 0)\right)=\frac{1}{2} A(0)$.

As we noted in (8.9), the extraction of $A(0)$ from the threshold photoproduction data is model dependent.

\section{CONCLUSIONS}

We have analyzed heavy meson photoproduction for all $\sqrt{s}$, using a bottom-up approach holographic construction. We have used the Witten diagrams in $\mathrm{AdS}_{5}$ for diffractive photoproduction of $J / \psi$, shown in Fig. 2, and explicitly computed the differential cross section for the heavy meson production, first near threshold, where it is dominated by the exchange of massive $2^{++}$glueballs as spin- 2 gravitons in bulk, and second away from threshold, where the exchange involves a tower of spin- $j$ states that transmute to the Pomeron. Our construction is general, and carries readily to heavier meson production such as $\Upsilon$. We have presented direct predictions for this production near and away from threshold. 
Our analysis allowed for the explicit derivation of all three holographic gravitational form factors $A(k), B(k)$, $C(k)$. In the double limit of a large number of colors and strong coupling, the holographic approach is dual to quenched QCD, so the form factors are mostly gluonic. Indeed, we have found that the form factors $A(k)$ and $D(k)=4 C(k)$ compare well to the gluonic gravitational form factors from the recent lattice QCD simulations [26]. The exception is the form factor $C(k)$ where a strong mixing on the lattice with the low-lying scalar-isoscalar was noted. We have used the $D(k)$ form factor to determined the distribution of the pressure and shear inside the proton. The results are comparable to those extracted recently from the lattice [28], and empirical data in [29].

We have found that the differential cross section for the photoproduction of heavy meson production is solely dependent on the invariant form factor $A(k)$ in our holographic analysis. The agreement of the differential and total cross sections with the recently reported GlueX data for $J / \Psi$ production near threshold [15] suggests that the heavy meson production is controlled by the tensor $2^{++}$glueball as a graviton exchange in bulk. Indeed, it is the graviton Regge trajectory at low $\sqrt{s}$ that transmutes to the Pomeron Regge trajectory at large $\sqrt{s}$ in holography, thereby providing a unified description of the photoproduction process of heavy mesons at all energies. These results complement those presented originally in [4,5] away from threshold, and are overall consistent with some of the general observations presented recently in $[17,18]$ close to threshold.

From a pertinent integral representation of $A(k)$ in the soft-wall model, we have determined the GPD of the gluons in the proton as probed by the spin-2 glueball or graviton near threshold, and the Pomeron way above threshold in the photoproduction process. The holographic construction clearly shows that the GlueX experiment [15] directly probes the tensor gluonic contribution of the energy form factor in the nucleon state as a bulk Dirac fermion. Conversely, we have used the GlueX data in combination with our holographic cross section result to extract in an almost model independent way the gravitational form factor $A(k)$ modulo $A(0)$, and consequently the gluon GPD of the proton. The value of $A(0)$ as it relates to the gluonic contribution to the proton spin is model dependent, and cannot be reliably extracted from the threshold data in the photoproduction process. Our observations are overall consistent with the original arguments presented in [19] using short distance QCD methods.

The forthcoming high statistics measurements from SoLID [16] will provide further insights and checks on the present holographic analysis.

\section{ACKNOWLEDGMENTS}

We thank Zein-Eddine Meziani for discussions. This work was supported by the U.S. Department of Energy under Contract No. DE-FG-88ER40388.

\section{APPENDIX A: WAVE FUNCTIONS AND PROPAGATORS IN HOLOGRAPHIC QCD}

\section{Dirac fermion/proton}

The normalized wave functions for the bulk Dirac fermion are [21]

$$
\begin{aligned}
& \Psi(p, z)=\psi_{R}(z) \Psi_{R}^{0}(p)+\psi_{L}(z) \Psi_{L}^{0}(p), \\
& \bar{\Psi}(p, z)=\psi_{R}(z) \bar{\Psi}_{R}^{0}(p)+\psi_{L}(z) \bar{\Psi}_{L}^{0}(p),
\end{aligned}
$$

where for the hard-wall

$$
\begin{aligned}
& \psi_{R}(z)=\frac{\sqrt{2} z^{5 / 2} J_{\tau-2}\left(m_{N} z\right)}{z_{0} J_{\tau-1}\left(m_{N} z_{0}\right)}, \\
& \psi_{L}(z)=\frac{\sqrt{2} z^{5 / 2} J_{\tau-1}\left(m_{N} z\right)}{z_{0} J_{\tau-1}\left(m_{N} z_{0}\right)},
\end{aligned}
$$

with the Bessel functions $J_{\alpha}\left(m_{N} z\right)$, and for the soft-wall

$$
\begin{aligned}
& \psi_{R}(z)=\frac{\tilde{n}_{R}}{\tilde{\kappa}_{N}^{\tau-2}} z^{\frac{5}{2} \xi^{\frac{\tau-2}{2}}} L_{0}^{(\tau-2)}(\xi), \\
& \psi_{L}(z)=\frac{\tilde{n}_{L}}{\tilde{\kappa}_{N}^{\tau-1}} z^{\frac{5}{2}} \xi^{\frac{\tau-1}{2}} L_{0}^{(\tau-1)}(\xi),
\end{aligned}
$$

with the generalized Laguerre polynomials $L_{n}^{(\alpha)}(\xi), \tilde{n}_{R}=$ $\tilde{n}_{L} \tilde{\kappa}_{N}^{-1} \sqrt{\tau-1}$, and $\tilde{n}_{L}=\tilde{\kappa}_{N}^{\tau} \sqrt{2 / \Gamma(\tau)}$. The bulk wave functions are normalized for the hard-wall as

$$
\int_{0}^{z_{0}} d z \sqrt{g} e_{a}^{\mu} \psi_{R / L}^{2}(z)=\delta_{a}^{\mu},
$$

and for the soft-wall as

$$
\int_{0}^{\infty} d z \sqrt{g} e^{-\phi} e_{a}^{\mu} \psi_{R / L}^{2}(z)=\delta_{a}^{\mu},
$$

with $\phi=\tilde{\kappa}_{N}^{2} z^{2}$, and the inverse vielbein $e_{a}^{\mu}=\sqrt{\left|g^{\mu \mu}\right|} \delta_{a}^{\mu}$ (no summation intended in $\mu$ ).

For both the hard-wall and soft-wall models, we have the twist parameter $\tau=3, \Psi_{R / L}^{0}(p)=P_{ \pm} u(p), \bar{\Psi}_{R / L}^{0}(p)=$ $\bar{u}(p) P_{\mp}$, and $P_{ \pm}=(1 / 2)\left(1 \pm \gamma^{5}\right)$. We also work with the normalizations of the boundary constant spinors for both the hard-wall and soft-wall models as

$$
\begin{aligned}
\bar{u}(p) u(p) & =2 m_{N}, \\
2 m_{N} \times \bar{u}\left(p^{\prime}\right) \gamma^{\mu} u(p) & =\bar{u}\left(p^{\prime}\right)\left(p^{\prime}+p\right)^{\mu} u(p) .
\end{aligned}
$$

\section{Photon/spin-1 mesons}

\section{a. Hard wall}

For timelike momenta $\left(q^{2}>0\right)$, the non-normalizable wave function for the virtual photon is generally given by $A_{\mu}=V(q, z) n_{\mu} e^{-i q \cdot x}$ where $[47,48]$ 


$$
V(q, z)=-g_{5} \sum_{n} \frac{F_{n} \phi_{n}(z)}{q^{2}-m_{n}^{2}}
$$

with $V(0, z)=V(q, 0)=1$, the decay constant of the vector mesons $F_{n}=\left.\left(1 / g_{5}\right)\left(-\frac{1}{z^{\prime}} \partial_{z^{\prime}} \phi_{n}\left(z^{\prime}\right)\right)\right|_{z^{\prime}=\epsilon}$, and the normalized wave functions of the vector mesons $A_{\mu}=\phi_{n}(z) n_{\mu} e^{-q \cdot x}$

$$
\phi_{n}(z)=c_{n} z J_{1}\left(m_{n} z\right) \equiv J_{A}\left(m_{n}, z\right)
$$

with $c_{n}=\frac{\sqrt{2}}{z_{0} J_{1}\left(m_{n} z_{0}\right)}$, which satisfy the normalization condition

$$
\int d z \sqrt{g}\left(g^{x x}\right)^{2} \phi_{n}(z) \phi_{m}(z)=\delta_{n m}
$$

In the hard-wall model, the summation in (A7) can be carried out analytically and is given by

$$
V(q, z)=\frac{\pi}{2} z q\left(\frac{Y_{0}\left(q z_{0}\right)}{J_{0}\left(q z_{0}\right)} J_{1}(q z)-Y_{1}(q z)\right) .
$$

For spacelike momenta $\left(q^{2}=-Q^{2}\right)$, the non-normalizable wave function for the virtual photon is generally given by $A_{\mu}=\mathcal{V}(Q, z) n_{\mu} e^{-q \cdot x}$ where

$$
\mathcal{V}(Q, z)=g_{5} \sum_{n} \frac{F_{n} \phi_{n}(z)}{Q^{2}+m_{n}^{2}}
$$

For example, in the hard-wall model, the summation in (A11) can be carried out analytically and is given by

$$
\mathcal{V}(Q, z)=Q z\left(\frac{K_{0}\left(Q z_{0}\right)}{I_{0}\left(Q z_{0}\right)} I_{1}(Q z)+K_{1}(Q z)\right),
$$

with the normalization $\mathcal{V}(0, z)=\mathcal{V}(Q, 0)=1$.

The bulk-to-bulk propagator for the massive mesons, for timelike momenta $\left(q^{2}>0\right)$, can be written as

$G_{\mu \nu}\left(z, z^{\prime}\right)=\mathcal{T}_{\mu \nu} G\left(z, z^{\prime}\right)=\left(-\eta_{\mu \nu}+\frac{k_{\mu} k_{\nu}}{m_{n}^{2}}\right) G\left(z, z^{\prime}\right)$

with

$$
G\left(z, z^{\prime}\right)=\sum_{n} \frac{\phi_{n}(z) \phi_{n}\left(z^{\prime}\right)}{q^{2}-m_{n}^{2}}
$$

For spacelike momenta $q^{2}=-Q^{2}$ in (A14). Also recall that $\mathcal{V}(q, z)=\left.\frac{1}{z^{\prime}} \partial_{z^{\prime}} G\left(z, z^{\prime}\right)\right|_{z^{\prime}=\epsilon}$. Note that for $z \rightarrow 0$, we can write (A14) as

$$
\begin{aligned}
G\left(z \rightarrow 0, z^{\prime}\right) & \approx \frac{\phi_{n}(z \rightarrow 0)}{-g_{5} F_{n}} \sum_{n} \frac{-g_{5} F_{n} \phi_{n}\left(z^{\prime}\right)}{q^{2}-m_{n}^{2}} \\
& =\frac{z^{2}}{2} \sum_{n} \frac{-g_{5} F_{n} \phi_{n}\left(z^{\prime}\right)}{q^{2}-m_{n}^{2}}=\frac{z^{2}}{2} V\left(q, z^{\prime}\right),
\end{aligned}
$$

where we used

$F_{n}=\left.\left(1 / g_{5}\right)\left(-\frac{1}{z^{\prime}} \partial_{z^{\prime}} \phi_{n}\left(z^{\prime}\right)\right)\right|_{z^{\prime}=\epsilon}=-\frac{1}{g_{5}} c_{n} m_{n}$,

and $\phi_{n}(z \rightarrow 0) \approx \frac{1}{2} c_{n} m_{n} z^{2}$ for the hard-wall. Defining the decay constant as $f_{n}=-\frac{F_{n}}{m_{n}}$, we have

$$
\phi_{n}(z)=\frac{f_{n}}{m_{n}} \times g_{5} m_{n} z J_{1}\left(m_{n} z\right),
$$

as required by vector meson dominance. For spacelike momenta $\left(q^{2}=-Q^{2}\right)$, we have

$G\left(z \rightarrow 0, z^{\prime}\right) \approx \frac{z^{2}}{2} \sum_{n} \frac{g_{5} F_{n} \phi_{n}\left(z^{\prime}\right)}{Q^{2}+m_{n}^{2}}=\frac{z^{2}}{2} \mathcal{V}\left(Q, z^{\prime}\right)$.

\section{b. Soft wall}

Similar relationships hold for the soft-wall model where the normalized wave function for vector mesons is given by [49]

$$
\phi_{n}(z)=c_{n} \tilde{\kappa}_{V}^{2} z^{2} L_{n}^{1}\left(\tilde{\kappa}_{V}^{2} z^{2}\right) \equiv J_{A}\left(m_{n}, z\right),
$$

with $c_{n}=\sqrt{2 / n+1}$ which is determined from the normalization condition (for the soft-wall model with background dilaton $\phi=\tilde{\kappa}_{V}^{2} z^{2}$ )

$$
\int d z \sqrt{g} e^{-\phi}\left(g^{x x}\right)^{2} \phi_{n}(z) \phi_{m}(z)=\delta_{n m}
$$

Therefore, we have

$$
F_{n}=\frac{1}{g_{5}}\left(-e^{-\phi} \frac{1}{z^{\prime}} \partial_{z^{\prime}} \phi_{n}\left(z^{\prime}\right)\right)_{z^{\prime}=\epsilon}=-\frac{2}{g_{5}} c_{n}(n+1) \tilde{\kappa}_{V}^{2}
$$

with $\phi_{n}(z \rightarrow 0) \approx c_{n} \tilde{\kappa}_{V}^{2} z^{2}(n+1)$. If we define the decay constant as $f_{n}=-F_{n} / m_{n}$, we have

$$
\phi_{n}(z)=\frac{f_{n}}{m_{n}} \times 2 g_{5} \tilde{\kappa}_{V}^{2} z^{2} L_{n}^{1}\left(\tilde{\kappa}_{V}^{2} z^{2}\right) \text {, }
$$

as required by vector meson dominance.

Note that for $z \rightarrow 0$, we can write the bulk-to-bulk propagator (A14) as 


$$
\begin{aligned}
G\left(z \rightarrow 0, z^{\prime}\right) & \approx \frac{\phi_{n}(z \rightarrow 0)}{-g_{5} F_{n}} \sum_{n} \frac{-g_{5} F_{n} \phi_{n}\left(z^{\prime}\right)}{q^{2}-m_{n}^{2}} \\
& =\frac{z^{2}}{2} \sum_{n} \frac{-g_{5} F_{n} \phi_{n}\left(z^{\prime}\right)}{q^{2}-m_{n}^{2}}=\frac{z^{2}}{2} V\left(q, z^{\prime}\right) .
\end{aligned}
$$

For spacelike momenta $\left(q^{2}=-Q^{2}\right)$, we have the bulkto-bulk propagator near the boundary

$G\left(z \rightarrow 0, z^{\prime}\right) \approx \frac{z^{2}}{2} \sum_{n} \frac{g_{5} F_{n} \phi_{n}\left(z^{\prime}\right)}{Q^{2}+m_{n}^{2}}=\frac{z^{2}}{2} \mathcal{V}\left(Q, z^{\prime}\right)$,

where [49]

$\mathcal{V}(Q, z)=\kappa_{V}^{2} z^{2} \int_{0}^{1} \frac{d x}{(1-x)^{2}} x^{a} \exp \left[-\frac{x}{1-x} \kappa_{V}^{2} z^{2}\right]$,

with the normalization $\mathcal{V}(0, z)=\mathcal{V}(Q, 0)=1$.

\section{Tansverse-traceless graviton/spin-2 glueballs}

\section{a. Hard wall}

For timelike momenta $\left(k^{2}>0\right)$, the non-normalizable wave function for the virtual tansverse-traceless graviton is generally given by $h_{\mu \nu}=h(k, z) \epsilon_{\mu \nu}^{T T} e^{-i k \cdot x}$ where [50]

$$
h(k, z)=-\sqrt{2} \kappa \sum_{n} \frac{F_{n} \psi_{n}(z)}{k^{2}-m_{n}^{2}},
$$

with the normalization $h(0, z)=h(k, 0)=1$, which could be relaxed. The decay constant of the spin-2 glueball with mass $m_{n}$ is

$$
F_{n}=\frac{1}{\sqrt{2} \kappa_{V}}\left(-\frac{1}{z^{\prime 3}} \partial_{z^{\prime}} \psi_{n}\left(z^{\prime}\right)\right)_{z^{\prime}=\epsilon}
$$

and the normalized wave functions of the spin-2 glueballs $h_{\mu \nu}=\psi_{n}(z) \epsilon_{\mu \nu}^{T T} e^{-i k \cdot x}$

$$
\psi_{n}(z)=c_{n} z^{2} J_{2}\left(m_{n} z\right) \equiv J_{h}\left(m_{n}, z\right),
$$

with $c_{n}=\frac{\sqrt{2}}{z_{0} J_{2}\left(m_{n} z_{0}\right)}$, which satisfy the normalization condition

$$
\int d z \sqrt{g}\left|g^{x x}\right| \psi_{n}(z) \psi_{m}(z)=\delta_{n m} .
$$

In the hard-wall model, the summation in (A26) can be carried out analytically and is given by $[21,48,50]$

$$
h(k, z)=\frac{\pi}{4} k^{2} z^{2}\left(\frac{Y_{1}\left(k z_{0}\right)}{J_{1}\left(k z_{0}\right)} J_{2}(k z)-Y_{2}(k z)\right) .
$$

For spacelike momenta $\left(k^{2}=-K^{2}\right)$, the non-normalizable wave function for the virtual transverse-traceless graviton is generally given by $h_{\mu \nu}=\mathcal{H}(K, z) \epsilon_{\mu \nu}^{T T} e^{-i k \cdot x}$ where

$$
\mathcal{H}(K, z)=\sqrt{2} \kappa \sum_{n} \frac{F_{n} \psi_{n}(z)}{K^{2}+m_{n}^{2}}
$$

In the hard-wall model, the summation in (A31) can be carried out analytically and is given by $[21,48,50]$

$\mathcal{H}(K, z)=\frac{1}{2} K^{2} z^{2}\left(\frac{K_{1}\left(K z_{0}\right)}{I_{1}\left(K z_{0}\right)} I_{2}(K z)+K_{2}(K z)\right)$.

For timelike momenta $\left(q^{2}>0\right)$, the bulk-to-bulk propagator for the massive spin-2 glueballs, can be written as $[21,48,50]$

$G_{\mu \nu \alpha \beta}^{T T}\left(z, z^{\prime}\right)=\frac{1}{2}\left(\mathcal{T}_{\mu \alpha} \mathcal{T}_{\nu \beta}+\mathcal{T}_{\mu \beta} \mathcal{T}_{\nu \alpha}-\frac{2}{3} \mathcal{T}_{\mu \nu} \mathcal{T}_{\alpha \beta}\right) G\left(z, z^{\prime}\right)$

with $\mathcal{T}_{\mu \nu}=-\eta_{\mu \nu}+k_{\mu} k_{\nu} / m_{n}^{2}$ and

$$
G\left(z, z^{\prime}\right)=\sum_{n} \frac{\psi_{n}(z) \psi_{n}\left(z^{\prime}\right)}{k^{2}-m_{n}^{2}} .
$$

For spacelike momenta, we simply replace $k^{2}=-K^{2}$ in (A34). Also remember that

$$
h(k, z)=\left.\frac{1}{z^{\prime 3}} \partial_{z^{\prime}} G\left(z, z^{\prime}\right)\right|_{z^{\prime}=\epsilon} .
$$

Note that for $z \rightarrow 0$, we can write (A34) as

$G\left(z \rightarrow 0, z^{\prime}\right) \approx \frac{z^{4}}{4} \sum_{n} \frac{-\sqrt{2} \kappa F_{n} \psi_{n}\left(z^{\prime}\right)}{k^{2}-m_{n}^{2}}=\frac{z^{4}}{4} h\left(k, z^{\prime}\right)$,

where we used

$F_{n}=\frac{1}{\sqrt{2} \kappa}\left(-\frac{1}{z^{\prime 3}} \partial_{z^{\prime}} \psi_{n}\left(z^{\prime}\right)\right)_{z^{\prime}=\epsilon}=-\frac{1}{2 \sqrt{2} \kappa} c_{n} m_{n}^{2}$,

and $\psi_{n}(z \rightarrow 0) \approx \frac{1}{8} c_{n} m_{n}^{2} z^{4}$ for the hard wall. Hence, for spacelike momenta $\left(k^{2}=-K^{2}\right)$, we have

$G\left(z \rightarrow 0, z^{\prime}\right) \approx \frac{z^{4}}{4} \sum_{n} \frac{\sqrt{2} \kappa_{V} F_{n} \phi_{n}\left(z^{\prime}\right)}{K^{2}+m_{n}^{2}}=\frac{z^{4}}{4} \mathcal{H}\left(K, z^{\prime}\right)$.

\section{b. Soft wall}

Similar relationships hold for the soft-wall model where the normalized wave function for spin-2 glueballs is given by [51] (note that the discussion in [51] is for general massive bulk scalar fluctuation but can be used for the spin-2 glueball which has an effective bulk action similar to massless bulk scalar fluctuation) 


$$
\psi_{n}(z)=c_{n} z^{4} L_{n}^{\Delta(j)-2}(2 \xi)
$$

with

$$
c_{n}=\left(\frac{2^{4} \tilde{\kappa}_{N}^{6} \Gamma(n+1)}{\Gamma(n+3)}\right)^{\frac{1}{2}}
$$

which is determined from the normalization condition (for soft-wall model with background dilaton $\phi=\tilde{\kappa}_{N}^{2} z^{2}$ )

$$
\int d z \sqrt{g} e^{-\phi}\left|g^{x x}\right| \psi_{n}(z) \psi_{m}(z)=\delta_{n m}
$$

Therefore we have

$F_{n}=\frac{1}{\sqrt{2} \kappa}\left(-\frac{1}{z^{\prime 3}} \partial_{z^{\prime}} \psi_{n}\left(z^{\prime}\right)\right)_{z^{\prime}=\epsilon}=-\frac{4}{\sqrt{2} \kappa} c_{n} L_{n}^{2}(0)$,

with $\psi_{n}(z \rightarrow 0) \approx c_{n} z^{4} L_{n}^{2}(0)$. For spacelike momenta $\left(q^{2}=-Q^{2}\right)$, we have the bulk-to-bulk propagator near the boundary

$G\left(z \rightarrow 0, z^{\prime}\right) \approx \frac{z^{4}}{4} \sum_{n} \frac{\sqrt{2} \kappa F_{n} \phi_{n}\left(z^{\prime}\right)}{K^{2}+m_{n}^{2}}=\frac{z^{4}}{4} \mathcal{H}\left(K, z^{\prime}\right)$,

where, for the soft-wall model $[21,48,51]$

$$
\begin{aligned}
\mathcal{H}(K, z) & =4 z^{4} \Gamma\left(a_{K}+2\right) U\left(a_{K}+2,3 ; 2 \xi\right) \\
& =\Gamma\left(a_{K}+2\right) U\left(a_{K},-1 ; 2 \xi\right) \\
& =\frac{\Gamma\left(a_{K}+2\right)}{\Gamma\left(a_{K}\right)} \int_{0}^{1} d x x^{a_{K}-1}(1-x) \exp \left(-\frac{x}{1-x}(2 \xi)\right),
\end{aligned}
$$

with $a_{K}=a / 2=K^{2} / 8 \tilde{\kappa}_{N}^{2}$, and we have used the transformation $U(m, n ; y)=y^{1-n} U(1+m-n, 2-n, y)$. Equation (A44) satisfies the normalization condition $\mathcal{H}(0, z)=\mathcal{H}(K, 0)=1$.

\section{Trace-full graviton/spin-0 glueballs}

\section{a. Hard wall}

For timelike momenta $\left(k^{2}>0\right)$, the non-normalizable wave function for the virtual trace-full graviton is generally given by $h_{\mu \nu}=k^{2} f(k, z) \epsilon_{\mu \nu}^{T} e^{-i k \cdot x}$ where

$$
f(k, z)=2 \sqrt{2} \kappa \sum_{n} \frac{F_{n} \psi_{n}(z)}{k^{2}-m_{n}^{2}},
$$

with $f(0, z)=f(k, 0)=1$, the decay constant of the spin-0 glueballs $F_{n}=\left.\frac{1}{2 \sqrt{2} \kappa}\left(\frac{1}{z^{\prime 3}} \partial_{z^{\prime}} \psi_{n}\left(z^{\prime}\right)\right)\right|_{z^{\prime}=\epsilon}$, and the normalized wave functions of the spin-0 glueballs $h_{\mu \nu}=\psi_{n}(z) \epsilon_{\mu \nu}^{T} e^{-k \cdot x}$ which satisfy the normalization condition

$$
\int d z \sqrt{g}\left|g^{x x}\right| \psi_{n}(z) \psi_{m}(z)=\delta_{n m}
$$

with the normalized wave functions for the spin- 0 glueballs

$$
\psi_{n}(z)=c_{n} z^{2} J_{2}\left(m_{n} z\right) \equiv J_{f}\left(m_{n}, z\right),
$$

where $c_{n}=\frac{\sqrt{2}}{z_{0} J_{2}\left(m_{n} z_{0}\right)}$. In the hard-wall model, the summation in (A45) can be carried out analytically and is given by

$$
f(k, z)=\frac{\pi}{4} k^{2} z^{2}\left(\frac{Y_{1}\left(k z_{0}\right)}{J_{1}\left(k z_{0}\right)} J_{2}(k z)-Y_{2}(k z)\right) .
$$

For spacelike momenta $\left(k^{2}=-K^{2}\right)$, the non-normalizable wave function for the virtual trace-full graviton is generally given by $h_{\mu \nu}=\mathcal{F}(K, z) \epsilon_{\mu \nu}^{T} e^{-k \cdot x}$ where

$$
\mathcal{F}(K, z)=-2 \sqrt{2} \kappa \sum_{n} \frac{F_{n} \psi_{n}(z)}{K^{2}+m_{n}^{2}} .
$$

The summation in (A49) can be carried out analytically and is given by

$\mathcal{F}(K, z)=\frac{1}{2} K^{2} z^{2}\left(\frac{K_{1}\left(K z_{0}\right)}{I_{1}\left(K z_{0}\right)} I_{2}(K z)+K_{2}(K z)\right)$.

For timelike momenta $\left(q^{2}>0\right)$, the bulk-to-bulk propagator for the massive spin-0 glueballs, can be written as $G_{\mu \nu \alpha \beta}^{T}\left(z, z^{\prime}\right)=\eta_{\mu \nu} \eta_{\alpha \beta} G\left(z, z^{\prime}\right)$ where

$$
G\left(z, z^{\prime}\right)=\sum_{n} \frac{\psi_{n}(z) \psi_{n}\left(z^{\prime}\right)}{k^{2}-m_{n}^{2}}
$$

with

$$
f(k, z)=\left(\frac{1}{z^{\prime 3}} \partial_{z^{\prime}} G\left(z, z^{\prime}\right)\right)_{z^{\prime}=\epsilon} .
$$

Note that for $z \rightarrow 0$, we can write (A51) as

$G\left(z \rightarrow 0, z^{\prime}\right) \approx \frac{z^{4}}{4} \sum_{n} \frac{2 \sqrt{2} \kappa F_{n} \psi_{n}\left(z^{\prime}\right)}{k^{2}-m_{n}^{2}}=\frac{z^{4}}{4} f\left(k, z^{\prime}\right)$,

where we used

$F_{n}=\frac{1}{2 \sqrt{2} \kappa}\left(\frac{1}{z^{\prime 3}} \partial_{z^{\prime}} G\left(z, z^{\prime}\right)\right)_{z^{\prime}=\epsilon}=\frac{1}{4 \sqrt{2} \kappa} c_{n} m_{n}^{2}$,

and $\psi_{n}(z \rightarrow 0) \approx \frac{1}{8} c_{n} m_{n}^{2} z^{4}$ for the hard wall. Hence, for spacelike momenta $\left(k^{2}=-K^{2}\right)$, we have

$G\left(z \rightarrow 0, z^{\prime}\right) \approx \frac{z^{4}}{4} \sum_{n} \frac{-2 \sqrt{2} \kappa F_{n} \phi_{n}\left(z^{\prime}\right)}{K^{2}+m_{n}^{2}}=\frac{z^{4}}{4} \mathcal{F}\left(K, z^{\prime}\right)$. 


\section{b. Soft wall}

Note that similar relationships hold for the trace-full graviton/spin-0 glueball in the soft-wall model. We do not detail them here as they are similar to the ones given for the spin-2 glueballs modilo normalization constants.

\section{Dilaton/spin-0 glueballs}

\section{a. Hard wall}

For timelike momenta $\left(k^{2}>0\right)$, the non-normalizable wave function for the virtual dilaton is generally given by

$$
\varphi(k, z)=\sqrt{2} \kappa \sum_{n} \frac{F_{n} \psi_{n}(z)}{k^{2}-m_{n}^{2}},
$$

with $\varphi(0, z)=\varphi(k, 0)=1$, the decay constant of the spin0 glueballs $F_{n}=\left.\frac{1}{\sqrt{2} \kappa}\left(\frac{1}{z^{\prime 3}} \partial_{z^{\prime}} \psi_{n}\left(z^{\prime}\right)\right)\right|_{z^{\prime}=\epsilon}$, and the normalized wave functions of the spin- 0 glueballs $\psi_{n}(z)$ which satisfy the normalization condition

$$
\int d z \sqrt{g}\left|g^{x x}\right| \psi_{n}(z) \psi_{m}(z)=\delta_{n m},
$$

with the normalized wave functions for the spin- 0 glueballs

$$
\psi_{n}(z)=c_{n} z^{2} J_{2}\left(m_{n} z\right) \equiv J_{\varphi}\left(m_{n}, z\right),
$$

where $c_{n}=\frac{\sqrt{2}}{z_{0} J_{2}\left(m_{n} z_{0}\right)}$. For example, in the hard-wall model, the summation in (A56) can be carried out analytically and is given by

$$
\varphi(k, z)=\frac{\pi}{4} k^{2} z^{2}\left(\frac{Y_{1}\left(k z_{0}\right)}{J_{1}\left(k z_{0}\right)} J_{2}(k z)-Y_{2}(k z)\right) .
$$

For spacelike momenta $\left(k^{2}=-K^{2}\right)$, the non-normalizable wave function for the virtual dilaton is generally given by

$$
\mathcal{D}(K, z)=-\sqrt{2} \kappa \sum_{n} \frac{F_{n} \psi_{n}(z)}{K^{2}+m_{n}^{2}} .
$$

For example, in the hard-wall model, the summation in (A60) can be carried out analytically and is given by

$$
\mathcal{D}(K, z)=\frac{1}{2} K^{2} z^{2}\left(\frac{K_{1}\left(K z_{0}\right)}{I_{1}\left(K z_{0}\right)} I_{2}(K z)+K_{2}(K z)\right) .
$$

For timelike momenta $\left(q^{2}>0\right)$, the bulk-to-bulk propagator for the massive spin- 0 glueballs, can be written as

$$
G\left(z, z^{\prime}\right)=\sum_{n} \frac{\psi_{n}(z) \psi_{n}\left(z^{\prime}\right)}{k^{2}-m_{n}^{2}}
$$

We recall that $\varphi(q, z)=\left.\frac{1}{z^{\prime 3}} \partial_{z^{\prime}} G\left(z, z^{\prime}\right)\right|_{z^{\prime}=\epsilon^{\prime}}$.

Note that for $z \rightarrow 0$, we can write (A62) as

$G\left(z \rightarrow 0, z^{\prime}\right) \approx \frac{z^{4}}{4} \sum_{n} \frac{\sqrt{2} \kappa F_{n} \psi_{n}\left(z^{\prime}\right)}{k^{2}-m_{n}^{2}}=\frac{z^{4}}{4} \varphi\left(k, z^{\prime}\right)$,

where we used

$$
F_{n}=\frac{1}{2 \sqrt{2} \kappa}\left(\frac{1}{z^{\prime 3}} \partial_{z^{\prime}} G\left(z, z^{\prime}\right)\right)_{z^{\prime}=\epsilon}=\frac{1}{4 \sqrt{2} \kappa} c_{n} m_{n}^{2},
$$

and $\psi_{n}(z \rightarrow 0) \approx \frac{1}{8} c_{n} m_{n}^{2} z^{4}$ for the hard wall. Hence, for spacelike momenta $\left(k^{2}=-K^{2}\right)$, we have

$G\left(z \rightarrow 0, z^{\prime}\right) \approx \frac{z^{4}}{4} \sum_{n} \frac{-2 \sqrt{2} \kappa F_{n} \phi_{n}\left(z^{\prime}\right)}{K^{2}+m_{n}^{2}}=\frac{z^{4}}{4} \mathcal{D}\left(K, z^{\prime}\right)$.

\section{b. Soft wall}

Note again, that similar relationships hold for the dilaton/ spin-0 glueballs in the soft-wall model, but we do not go into details here as it is very similar to the spin-2 glueballs up to normalization constants.

\section{APPENDIX B: CONTRIBUTIONS TO HOLOGRAPHIC PHOTOPRODUCTION}

Here most of the results will be given for the soft-wall model explicitly. The results for the hard-wall model follow by setting $\phi=0$.

\section{Dilaton contribution}

The dilaton contribution to the holographic photoproduction amplitude can be determined from Fig. 2 by replacing the spin-2 glueball propagator by spin- 0 glueball propagator of a dilaton as

$$
\begin{aligned}
i \mathcal{A}_{A p \rightarrow A p}^{\varphi}(s, t) & =\sum_{n} i \tilde{\mathcal{A}}_{A p \rightarrow A p}^{\varphi}\left(m_{n}, s, t\right), \\
i \tilde{\mathcal{A}}_{A p \rightarrow A p}^{\varphi}\left(m_{n}, s, t\right) & =(-i) V_{\varphi A A}\left(q_{1}, q_{2}, k, m_{n}\right) \times \tilde{G}_{\varphi}\left(m_{n}, t\right) \times(-i) V_{\varphi \bar{\Psi} \Psi}\left(p_{1}, p_{2}, k, m_{n}\right),
\end{aligned}
$$

with the bulk vertices $\left(k=p_{2}-p_{1}=q_{1}-q_{2}\right)$ 


$$
\begin{aligned}
V_{\varphi A A}\left(q, q^{\prime}, k, m_{n}\right) & \equiv\left(\frac{\delta S_{\varphi A A}^{k}}{\delta \varphi(k, z)}\right) J_{\varphi}\left(m_{n}, z\right)=\sqrt{2 \kappa^{2}} \times \frac{1}{4} \int d z \sqrt{g} e^{-\phi} z^{4} K\left(q, q^{\prime}, n, n^{\prime}, z\right) J_{\varphi}\left(m_{n}, z\right), \\
V_{\varphi \bar{\Psi} \Psi}\left(p_{1}, p_{2}, k, m_{n}\right) & \equiv\left(\frac{\delta S_{\varphi \bar{\Psi} \Psi}^{k}}{\delta\left(\partial_{z} \varphi(k, z)\right)}\right) \partial_{z} J_{\varphi}\left(m_{n}, z\right)+\left(\frac{\delta S_{\varphi \bar{\Psi} \Psi}^{k}}{\delta(\varphi(k, z))}\right) J_{\varphi}\left(m_{n}, z\right) \\
& =\frac{\sqrt{2 \kappa^{2}}}{2} \int d z \sqrt{g} e^{-\phi} z \bar{\Psi}\left(p_{2}, z\right)\left(\gamma^{5} \partial_{z} J_{\varphi}\left(m_{n}, z\right)+k_{\alpha} \gamma^{\alpha} J_{\varphi}\left(m_{n}, z\right)\right) \Psi\left(p_{1}, z\right),
\end{aligned}
$$

and the bulk-to-bulk propagator

$$
\begin{aligned}
G_{\varphi}\left(m_{n}, t, z, z^{\prime}\right) & =J_{\varphi}\left(m_{n}, z\right) \tilde{G}_{\varphi}\left(m_{n}, t\right) J_{\varphi}\left(m_{n}, z^{\prime}\right), \\
\tilde{G}_{\varphi}\left(m_{n}, t\right) & =\frac{i}{t-m_{n}^{2}+i \epsilon} .
\end{aligned}
$$

For $z^{\prime} \rightarrow 0$, and $t=-K^{2}$ in (B3), we can use (A65), which simplifies (B1) as

$$
\begin{aligned}
i \mathcal{A}_{A p \rightarrow A p}^{\varphi}(s, t) & \approx(-i) \mathcal{V}_{\varphi A A}\left(q_{1}, q_{2}, k\right) \times(i) \times(-i) \mathcal{V}_{\varphi \bar{\Psi} \Psi}\left(p_{1}, p_{2}, k\right), \\
\mathcal{V}_{\varphi A A}\left(q_{1}, q_{2}, k\right) & =\sqrt{2 \kappa^{2}} \times \sqrt{\frac{1}{4}} \int d z \sqrt{g} e^{-\phi} z^{4} K\left(q, q^{\prime}, n, n^{\prime}, z\right) \frac{z^{4}}{4}, \\
\mathcal{V}_{\varphi \bar{\Psi} \Psi}\left(p_{1}, p_{2}, k\right) & =\sqrt{2 \kappa^{2}} \times \frac{1}{2} \int d z e^{-\phi} \sqrt{g} z \bar{\Psi}\left(p_{2}, z\right)\left(\gamma^{5} \partial_{z} \mathcal{D}(K, z)+k_{\alpha} \gamma^{\alpha} \mathcal{D}(K, z)\right) \Psi\left(p_{1}, z\right) .
\end{aligned}
$$

\section{Graviton contribution}

The graviton contribution in Fig. 2 in the diffractive part of the holographic photoproduction amplitude was analyzed in [52] for the Pomeron kinematics in the hard-wall model. Here we will give the results for all kinematics for both the CFT case in AdS, and the conformally broken case in walled AdS.

In AdS space, for the tansverse-traceless part, Witten's diagrammatic rules give formally

$$
\begin{aligned}
i \mathcal{A}_{A p \rightarrow A p}^{h}(s, t) & =\sum_{n} i \tilde{\mathcal{A}}_{A p \rightarrow A p}^{h}\left(m_{n}, s, t\right), \\
i \tilde{\mathcal{A}}_{A \rightarrow A p}^{h}\left(m_{n}, s, t\right) & =(-i) V_{h A A}^{\mu \nu(T T)}\left(q, q^{\prime}, k, m_{n}\right) \times \tilde{G}_{\mu \nu \alpha \beta}^{T T}\left(m_{n}, t\right) \times(-i) V_{h \bar{\Psi} \Psi}^{\alpha \beta(T T)}\left(p_{1}, p_{2}, k, m_{n}\right), \\
i \mathcal{A}_{A p \rightarrow A p}^{f}(s, t) & =\sum_{n} i \tilde{\mathcal{A}}_{A p \rightarrow A p}^{f}\left(m_{n}, s, t\right), \\
i \tilde{\mathcal{A}}_{A p \rightarrow A p}^{f}\left(m_{n}, s, t\right) & =(-i) V_{f A A}^{\mu \nu(T)}\left(q, q^{\prime}, k, m_{n}\right) \times \tilde{G}_{\mu \nu \alpha \beta}^{T}\left(m_{n}, t\right) \times(-i) V_{f \bar{\Psi} \Psi}^{\alpha \beta(T)}\left(p_{1}, p_{2}, k, m_{n}\right),
\end{aligned}
$$

with the bulk vertices $\left(k=p_{2}-p_{1}=q-q^{\prime}\right)$

$$
\begin{aligned}
V_{h A A}^{\mu \nu(T T)}\left(q, q^{\prime}, k, m_{n}\right) & \equiv\left(\frac{\delta S_{h A A}^{k}}{\delta\left(\epsilon_{\mu \nu}^{T T} h(k, z)\right)}\right) J_{h}\left(m_{n}, z\right)=\sqrt{2 \kappa^{2}} \times \frac{1}{2} \int d z \sqrt{g} e^{-\phi} z^{4} K^{\mu \nu}\left(q, q^{\prime}, n, n^{\prime}, z\right) J_{h}\left(m_{n}, z\right) \\
V_{h \bar{\Psi} \Psi}^{\alpha \beta(T T)}\left(p_{1}, p_{2}, k, m_{n}\right) & \equiv\left(\frac{\delta S_{h \bar{\Psi} \Psi}^{k}}{\delta\left(\epsilon_{\alpha \beta}^{T T} h(k, z)\right)}\right) J_{h}\left(m_{n}, z\right)=-\sqrt{2 \kappa^{2}} \times \frac{1}{2} \int d z \sqrt{g} e^{-\phi} z \bar{\Psi}\left(p_{2}, z\right) \gamma^{\alpha} p^{\beta} \Psi\left(p_{1}, z\right) J_{h}\left(m_{n}, z\right) \\
V_{f A A}^{\mu \nu(T)}\left(q, q^{\prime}, k, m_{n}\right) & \equiv\left(\frac{\delta S_{f A A}^{k}}{\delta\left(\epsilon_{\mu \nu}^{T} f(k, z)\right)}\right) J_{f}\left(m_{n}, z\right) \\
& =\sqrt{2 \kappa^{2}} \times \frac{1}{4} \int d z \sqrt{g} e^{-\phi} z^{4} \tilde{k}^{2}\left(K^{\mu \nu}\left(q, q^{\prime}, n, n^{\prime}, z\right)-\frac{1}{4} \eta^{\mu \nu} K\left(q, q^{\prime}, n, n^{\prime}, z\right)\right) J_{f}\left(m_{n}, z\right), \\
V_{f \bar{\Psi} \Psi}^{\alpha \beta(T)}\left(p_{1}, p_{2}, k, m_{n}\right) & \equiv\left(\frac{\delta S_{f \bar{\Psi} \Psi}^{k}}{\delta \partial_{z}\left(\epsilon_{\alpha \beta}^{T} f(k, z)\right)}\right) \partial_{z} J_{f}\left(m_{n}, z\right)+\left(\frac{\delta S_{f \bar{\Psi} \Psi}^{k}}{\delta\left(\epsilon_{\alpha \beta}^{T} f(k, z)\right)}\right) J_{f}\left(m_{n}, z\right) \\
& =-\sqrt{2 \kappa^{2}} \times \frac{1}{2} \int d z \sqrt{g} e^{-\phi} z \tilde{k}^{2} \bar{\Psi}\left(p_{2}, z\right)\left(\eta^{\alpha \beta} \gamma^{5} \partial_{z} J_{f}\left(m_{n}, z\right)+\gamma^{\alpha} p^{\beta} J_{f}\left(m_{n}, z\right)+\eta^{\alpha \beta} k_{\mu} \gamma^{\mu} J_{f}\left(m_{n}, z\right)\right) \Psi\left(p_{1}, z\right),
\end{aligned}
$$


with $p=\left(p_{1}+p_{2}\right) / 2$. The bulk-to-bulk graviton propagator is $G_{\mu \nu \alpha \beta}=G_{\mu \nu \alpha \beta}^{T T}+G_{\mu \nu \alpha \beta}^{T}$. The transverse and traceless TTpart describes massive $2^{++}$glueballs $[53,54]$

$$
\begin{aligned}
G_{\mu \nu \alpha \beta}^{T T}\left(m_{n}, t, z, z^{\prime}\right) & =J_{h}\left(m_{n}, z\right) \tilde{G}_{\mu \nu \alpha \beta}^{T T}\left(m_{n}, t\right) J_{h}\left(m_{n}, z^{\prime}\right) \\
\tilde{G}_{\mu \nu \alpha \beta}^{T T}\left(m_{n}, t\right) & =\frac{1}{2}\left(\mathcal{T}_{\mu \alpha} \mathcal{T}_{\nu \beta}+\mathcal{T}_{\mu \beta} \mathcal{T}_{\nu \alpha}-\frac{2}{3} \mathcal{T}_{\mu \nu} \mathcal{T}_{\alpha \beta}\right) \frac{i}{t-m_{n}^{2}+i \epsilon},
\end{aligned}
$$

with $\tilde{G}$ the boundary propagator,

$$
\mathcal{T}_{\mu \nu}=-\eta_{\mu \nu}+k_{\mu} k_{\nu} / m_{n}^{2}
$$

The trace-full T-part $G_{\mu \nu \alpha \beta}^{T}$ describes massive $0^{++}$glueballs [54]

$$
G_{\mu \nu \alpha \beta}^{T}\left(m_{n}, t, z, z^{\prime}\right)=J_{f}\left(m_{n}, z\right) \tilde{G}_{\mu \nu \alpha \beta}^{T}\left(m_{n}, t\right) J_{f}\left(m_{n}, z^{\prime}\right),
$$

with the boundary propagator

$$
\tilde{G}_{\mu \nu \alpha \beta}^{T}\left(m_{n}, t\right)=\eta_{\mu \nu} \eta_{\alpha \beta} \frac{i}{t-m_{n}^{2}+i \epsilon} .
$$

For $z^{\prime} \rightarrow 0$, and $t=-K^{2}$, and $t=-K^{2}$ in (B7) and (B8). We can use (A38) and (A55), and simplify (B5) as

$$
\begin{aligned}
& i \mathcal{A}_{A p \rightarrow A p}^{h}(s, t) \approx(-i) \mathcal{V}_{h A A}^{\mu \nu(T T)}\left(q_{1}, q_{2}, k_{z}\right) \times\left(\frac{i}{2} \eta_{\mu \alpha} \eta_{\nu \beta}\right) \times(-i) \mathcal{V}_{h \Psi \Psi}^{\alpha \beta(T T)}\left(p_{1}, p_{2}, k_{z}\right), \\
& i \mathcal{A}_{A p \rightarrow A p}^{f}(s, t) \approx(-i) \mathcal{V}_{h A A}^{\mu \nu(T)}\left(q_{1}, q_{2}, k\right) \times\left(i \eta_{\mu \nu} \eta_{\alpha \beta}\right) \times(-i) \mathcal{V}_{f \bar{\Psi} \Psi}^{\alpha \beta(T)}\left(p_{1}, p_{2}, k\right),
\end{aligned}
$$

with

$$
\begin{aligned}
\mathcal{V}_{h A A}^{\mu \nu(T T)}\left(q_{1}, q_{2}, k_{z}\right) & =\sqrt{2 \kappa^{2}} \times \frac{1}{2} \int d z \sqrt{g} e^{-\phi} z^{4} K^{\mu \nu}\left(q, q^{\prime}, n, n^{\prime}, z\right) \frac{z^{4}}{4} \\
\mathcal{V}_{h \bar{\Psi} \Psi}^{\alpha \beta(T T)}\left(p_{1}, p_{2}, k_{z}\right) & =-\sqrt{2 \kappa^{2}} \times \frac{1}{2} \int d z \sqrt{g} e^{-\phi} z \bar{\Psi}\left(p_{2}, z\right) \gamma^{\mu} p^{\nu} \Psi\left(p_{1}, z\right) \mathcal{H}(K, z) \\
\mathcal{V}_{f A A}^{\mu \nu(T)}\left(q_{1}, q_{2}, k\right) & =\sqrt{2 \kappa^{2}} \times \frac{1}{4} \int d z \sqrt{g} e^{-\phi} z^{4} \tilde{k}^{2}\left(K^{\mu \nu}\left(q, q^{\prime}, n, n^{\prime}, z\right)-\frac{1}{4} \eta^{\mu \nu} K\left(q, q^{\prime}, n, n^{\prime}, z\right)\right) \frac{z^{4}}{4} \\
\mathcal{V}_{f \bar{\Psi} \Psi}^{\alpha \beta(T)}\left(p_{1}, p_{2}, k\right) & =-\sqrt{2 \kappa^{2}} \times \frac{1}{2} \times \frac{1}{2} \int d z \sqrt{g} e^{-\phi} z \tilde{k}^{2} \bar{\Psi}\left(p_{2}, z\right)\left(\eta^{\alpha \beta} \gamma^{5} \partial_{z} \mathcal{F}(K, z)+\gamma^{\alpha} p^{\beta} \mathcal{F}(K, z)+\eta^{\alpha \beta} k_{\mu} \gamma^{\mu} \mathcal{F}(K, z)\right) \Psi\left(p_{1}, z\right)
\end{aligned}
$$

\section{APPENDIX C: ELEMENTS OF THE REGGEIZATION}

\section{Hard wall}

The reggeization of the graviton exchange is obtained through the substitution [55]

$$
J_{h}\left(m_{n}(j), z\right) \rightarrow \tilde{\psi}_{n}(j, z)=z^{-(j-2)} \psi_{n}(j, z)
$$

followed by the summation over all spin-j exchanges using the Sommerfeld-Watson formula

$$
\frac{1}{2} \sum_{j \geq 2}\left(s^{j}+(-s) j\right) \rightarrow-\frac{\pi}{2} \int_{\mathbb{C}} \frac{d j}{2 \pi i}\left(\frac{s^{j-2}+(-s)^{j-2}}{\sin \pi j}\right)
$$


for a pertinent choice of the contour $\mathbb{C}$. This requires the analytical continuation of the exchanged amplitudes to the complex j-plane. For the hard-wall model, the normalized wave function is given by

$$
\begin{aligned}
\psi_{n}(j, z) & =c_{n}(j) z^{2} J_{\tilde{\Delta}(j)}\left(m_{n}(j) z\right) \\
c_{n}(j) & =\frac{1}{\sqrt{2} z_{0} J_{\tilde{\Delta}(j)}\left(m_{n}(j) z_{0}\right)}
\end{aligned}
$$

for $\partial_{z} \psi_{n}\left(j, z_{0}\right)=0$ and

$$
\tilde{\Delta}(j) \equiv \Delta(j)-2=(4+2 \sqrt{\lambda}(j-2))^{\frac{1}{2}}=\sqrt{2 \sqrt{\lambda}\left(j-j_{0}\right)}
$$

with $j_{0}=2-\frac{2}{\sqrt{\lambda}}$ and $j \geq 2$.

For timelike momenta $k^{2}>0$, we can also determine the non-normalizable wave function for the virtual tansversetraceless spin-j glueball, as

$$
h(j, k, z)=-\sqrt{2} \kappa \sum_{n} \frac{F_{n}(j) \psi_{n}(j, z)}{k^{2}-m_{n}^{2}(j)},
$$

which satisfies the boundary conditions $\partial_{z} h\left(j=2, k, z_{0}\right)=$ 0 . We define a decay constant function (not exactly the decay constant) of the spin-j glueballs as

$$
\left.F_{n}(j) \equiv \frac{C(j, k, \epsilon)}{\sqrt{2} \kappa}\left(-\sqrt{g} e^{-\phi}\left|g^{x x}\right| \partial_{z^{\prime}} \psi_{n}\left(j, z^{\prime}\right)\right)\right|_{z^{\prime}=\epsilon} .
$$

The normalized wave functions of the spin- $j$ glueballs $\psi_{n}(j, z)$ satisfy the normalization condition

$$
\int d z \sqrt{g} e^{-\phi}\left|g^{x x}\right| \psi_{n}(j, z) \psi_{m}(j, z)=\delta_{n m} .
$$

For example, in the hard-wall model, the summation over $n$ in (C5) can be carried out analytically and is given by

$$
\begin{aligned}
h(j, k, z) & =-\sqrt{2} \kappa \sum_{n} \frac{F_{n}(j) \psi_{n}(j, z)}{k^{2}-m_{n}^{2}(j)} \\
& =z^{2}\left(\frac{A\left(j, k, z_{0}\right)}{B\left(j, k, z_{0}\right)} J_{\tilde{\Delta}(j)}(k z)-Y_{\tilde{\Delta}(j)}(k z)\right),
\end{aligned}
$$

with

$$
\begin{aligned}
& A\left(j, k, z_{0}\right)=\left.\partial_{z}\left(z^{2} Y_{\tilde{\Delta}(j)}(k z)\right)\right|_{z=z_{0}}, \\
& B\left(j, k, z_{0}\right)=\left.\partial_{z}\left(z^{2} J_{\tilde{\Delta}(j)}(k z)\right)\right|_{z=z_{0}}
\end{aligned}
$$

We also define

$$
C(j, k, \epsilon)=h(j, k, \epsilon) \approx-\epsilon^{2} Y_{\tilde{\Delta}(j)}(k \epsilon) .
$$

For spacelike momenta $k^{2}=-K^{2}$, the non-normalizable wave function for the virtual transverse-traceless graviton is generally given by

$$
\mathcal{H}(j, K, z)=\sqrt{2} \kappa \sum_{n} \frac{\mathcal{F}_{n}(j) \psi_{n}(j, z)}{K^{2}+m_{n}^{2}(j)},
$$

which satisfies the IR boundary conditions $\partial_{z} \mathcal{H}\left(j=2, K, z_{0}\right)=$ 0 . We have defined a decay constant function (for spacelike momenta) of the spin-j glueballs as

$$
\mathcal{F}_{n}(j) \equiv-\left.\frac{\mathcal{C}(j, k, \epsilon)}{\sqrt{2} \kappa}\left(-\sqrt{g} e^{-\phi}\left|g^{x x}\right| \partial_{z^{\prime}} \psi_{n}\left(j, z^{\prime}\right)\right)\right|_{z^{\prime}=\epsilon} .
$$

In the hard-wall model, the summation in (C11) reduces to

$$
\begin{aligned}
\mathcal{H}(j, K, z) & =\sqrt{2} \kappa \sum_{n} \frac{\mathcal{F}_{n}(j) \psi_{n}(j, z)}{K^{2}+m_{n}^{2}(j)} \\
& =z^{2}\left(\frac{\mathcal{A}\left(j, K, z_{0}\right)}{\mathcal{B}\left(j, K, z_{0}\right)} I_{\tilde{\Delta}(j)}(K z)+K_{\tilde{\Delta}(j)}(K z)\right),
\end{aligned}
$$

with

$$
\begin{aligned}
& \mathcal{A}\left(j, K, z_{0}\right)=\left.\partial_{z}\left(z^{2} K_{\tilde{\Delta}(j)}(K z)\right)\right|_{z=z_{0}}, \\
& \mathcal{B}\left(j, K, z_{0}\right)=\left.\partial_{z}\left(z^{2} I_{\tilde{\Delta}(j)}(K z)\right)\right|_{z=z_{0}} .
\end{aligned}
$$

We also define

$$
\mathcal{C}(j, K, \epsilon)=\mathcal{H}(j, K, \epsilon) \approx \epsilon^{2} K_{\tilde{\Delta}(j)}(K \epsilon) .
$$

For timelike momenta $k^{2}>0$, the bulk-to-bulk propagator for the massive spin-j glueballs, can be written as

$$
\begin{aligned}
\bar{G}_{\mu \nu \alpha \beta}^{T T}\left(j, z, z^{\prime}\right)= & \frac{1}{2}\left(\mathcal{T}_{\mu \alpha}(j) \mathcal{T}_{\nu \beta}(j)+\mathcal{T}_{\mu \beta}(j) \mathcal{T}_{\nu \alpha}(j)\right. \\
& \left.-\frac{2}{3} \mathcal{T}_{\mu \nu}(j) \mathcal{T}_{\alpha \beta}(j)\right) \bar{G}\left(j, z, z^{\prime}\right), \\
\bar{G}\left(j, z, z^{\prime}\right)= & z^{-(j-2)} G\left(j, z, z^{\prime}\right) z^{\prime-(j-2)} \\
= & \sum_{n} \frac{\tilde{\psi}_{n}(j, z) \tilde{\psi}_{n}\left(j, z^{\prime}\right)}{k^{2}-m_{n}^{2}(j)}
\end{aligned}
$$

and $\mathcal{T}_{\mu \nu}(j)=-\eta_{\mu \nu}+k_{\mu} k_{\nu} / m_{n}^{2}(j)$. For spacelike momenta, we simply replace $k^{2}=-K^{2}$ in (C16). Also remember that

$h(j, k, z)=\left.C(j, k, \epsilon) \sqrt{g} e^{-\phi}\left|g^{x x}\right| \partial_{z^{\prime}} G\left(j, z, z^{\prime}\right)\right|_{z^{\prime}=\epsilon}$.

Note that for $z \rightarrow 0$, we can approximately write the bulkto-bulk propagator $G\left(j, z, z^{\prime}\right)=\sum_{n} \frac{\psi_{n}(z) \psi_{n}\left(z^{\prime}\right)}{k^{2}-m_{n}^{2}(j)}$ in (C16) in terms of the unnormalized bulk-to-boundary propagator $h\left(j, k, z^{\prime}\right)$ as 


$$
G\left(j, z \rightarrow 0, z^{\prime}\right) \approx \frac{\psi_{n}(z \rightarrow 0)}{(-\sqrt{2} \kappa) F_{n}(j)} \times(-\sqrt{2} \kappa) \sum_{n} \frac{F_{n}(j) \psi_{n}\left(z^{\prime}\right)}{k^{2}-m_{n}^{2}(j)}=\frac{2^{-\tilde{\Delta}(j)} \times k^{\tilde{\Delta}(j)} \times z^{\tilde{\Delta}(j)+2}}{\tilde{\Delta}(j)+2} h\left(j, k, z^{\prime}\right),
$$

where we used

$$
F_{n}(j)=\left.\frac{C(j, k, \epsilon)}{\sqrt{2} \kappa}\left(-\sqrt{g} e^{-\phi}\left|g^{x x}\right| \partial_{z^{\prime}} \psi_{n}\left(j, z^{\prime}\right)\right)\right|_{z^{\prime}=\epsilon}=-\frac{1}{\sqrt{2} \kappa} \frac{2^{\tilde{\Delta}(j)}}{\pi} c_{n}(j)\left(\frac{m_{n}(j)}{k}\right)^{\tilde{\Delta}(j)}(2+\tilde{\Delta}(j)) \frac{\Gamma(\tilde{\Delta}(j))}{\Gamma(1+\tilde{\Delta}(j))},
$$

with $\psi_{n}(z \rightarrow 0) \approx \frac{2^{-\tilde{\Delta}(j)}}{\Gamma[1+\tilde{\Delta}(j)]} c_{n}(j)\left(m_{n}\right)^{\tilde{\Delta}(j)} z^{\tilde{\Delta}(j)+2}$, and $C(j, k, \epsilon)=\frac{1}{k^{2}}(k \epsilon)^{2-\tilde{\Delta}(j)} \frac{2^{\tilde{\Delta}(j)}}{\pi} \Gamma(\tilde{\Delta}(j)$ for the hard wall.

For spacelike momenta $k^{2}>0$, we also have

$$
G\left(j, z \rightarrow 0, z^{\prime}\right) \approx \frac{\psi_{n}(z \rightarrow 0)}{(\sqrt{2} \kappa) \mathcal{F}_{n}(j)} \times(\sqrt{2} \kappa) \sum_{n} \frac{\mathcal{F}_{n}(j) \psi_{n}\left(z^{\prime}\right)}{K^{2}+m_{n}^{2}(j)}=\frac{\frac{2^{1-\tilde{\Delta}(j)}}{\pi} \times K^{\tilde{\Delta}(j)} \times z^{\tilde{\Delta}(j)+2}}{\tilde{\Delta}(j)+2} \mathcal{H}\left(j, K, z^{\prime}\right),
$$

where we used

$$
\mathcal{F}_{n}(j)=-\left.\frac{\mathcal{C}(j, K, \epsilon)}{\sqrt{2} \kappa}\left(-\sqrt{g} e^{-\phi}\left|g^{x x}\right| \partial_{z^{\prime}} \psi_{n}\left(j, z^{\prime}\right)\right)\right|_{z^{\prime}=\epsilon}=\frac{1}{\sqrt{2} \kappa} 2^{\tilde{\Delta}(j)-1} c_{n}(j)\left(\frac{m_{n}(j)}{K}\right)^{\tilde{\Delta}(j)}(2+\tilde{\Delta}(j)) \frac{\Gamma(\tilde{\Delta}(j))}{\Gamma(1+\tilde{\Delta}(j))},
$$

with

$$
\begin{aligned}
\psi_{n}(z \rightarrow 0) & \approx \frac{2^{-\tilde{\Delta}(j)}}{\Gamma[1+\tilde{\Delta}(j)]} c_{n}(j)\left(m_{n}\right)^{\tilde{\Delta}(j)} z^{\tilde{\Delta}(j)+2}, \\
\mathcal{C}(j, K, \epsilon) & =\frac{1}{K^{2}}(K \epsilon)^{2-\tilde{\Delta}(j)} 2^{\tilde{\Delta}(j)-1} \Gamma(\tilde{\Delta}(j))
\end{aligned}
$$

for the hard-wall model. Also remember that

$$
\mathcal{H}(j, K, z)=-\left.\mathcal{C}(j, K, \epsilon) \sqrt{g} e^{-\phi}\left|g^{x x}\right| \partial_{z^{\prime}} G\left(j, z, z^{\prime}\right)\right|_{z^{\prime}=\epsilon} .
$$

\section{Soft wall}

The same relationships hold for the soft-wall model, where the spin-j glueballs' normalized wave functions are given in terms of the generalized Laguerre polynomials as [51] (note that the discussion in [51] is for general massive bulk scalar fluctuations but can be used for spin-j glueballs which have an effective bulk action (or bulk equation of motion) similar to massive bulk scalar fluctuations [55])

$$
\psi_{n}(j, z)=c_{n}(j) z^{\Delta} L_{n}^{\Delta(j)-2}(2 \xi),
$$

where $\xi=\tilde{\kappa}_{N}^{2} z^{2}$, and the normalization coefficients are

$$
c_{n}(j)=\left(\frac{2^{\Delta(j)} \tilde{\kappa}_{N}^{2(\Delta(j)-1)} \Gamma(n+1)}{\Gamma(n+\Delta(j)-1)}\right)^{\frac{1}{2}} .
$$

The non-normalized bulk-to-boundary propagators for spin-j glueballs are given in terms of Kummer's (confluent hypergeometric) function of the second kind and its integral representation as (for spacelike momenta $k^{2}=-K^{2}$ )

$$
\begin{aligned}
\mathcal{H}(j, K, z)= & z^{\Delta} U\left(a_{K}+\frac{\Delta(j)}{2}, \Delta(j)-1 ; 2 \xi\right) \\
= & z^{\Delta(j)}(2 \xi)^{2-\Delta(j)} U(\tilde{a}(j), \tilde{b}(j) ; 2 \xi) \\
= & z^{\Delta(j)}(2 \xi)^{2-\Delta(j)} \frac{1}{\Gamma(\tilde{a}(j))} \int_{0}^{1} d x x^{\tilde{a}(j)-1}(1-x)^{-\tilde{b}(j)} \\
& \times \exp \left(-\frac{x}{1-x}(2 \xi)\right), \quad \text { (C26) }
\end{aligned}
$$

where

$$
\begin{aligned}
a_{K} & =\frac{a}{2}=\frac{K^{2}}{8 \tilde{\kappa}_{N}^{2}}, \\
\tilde{a}(j) & =a_{K}+2-\frac{\Delta(j)}{2}, \\
\tilde{b}(j) & =3-\Delta(j),
\end{aligned}
$$

and we have used the transformation $U(m, n ; y)=y^{1-n} U(1+$ $m-n, 2-n, y)$. The bulk-to-bulk propagator can also be approximated as (for spacelike momenta $k^{2}=-K^{2}$ )

$$
\begin{aligned}
G\left(j, z \rightarrow 0, z^{\prime}\right) & \approx \frac{\psi_{n}(z \rightarrow 0)}{(\sqrt{2} \kappa) \mathcal{F}_{n}(j)} \times(\sqrt{2} \kappa) \sum_{n} \frac{\mathcal{F}_{n}(j) \psi_{n}\left(z^{\prime}\right)}{K^{2}+m_{n}^{2}(j)} \\
& =\frac{\frac{2^{\Delta(j)-2} \Gamma\left(a_{K}+\frac{\Delta(j)}{2}\right)}{\Gamma(\Delta(j)-2)} \times \tilde{\kappa}_{N}^{2 \Delta(j)-4} \times z^{\Delta(j)}}{\Delta(j)} \mathcal{H}\left(j, K, z^{\prime}\right),
\end{aligned}
$$

where we used

$$
\mathcal{F}_{n}(j)=-\left.\frac{\mathcal{C}(j, K, \epsilon)}{\sqrt{2} \kappa}\left(-\sqrt{g} e^{-\phi}\left|g^{x x}\right| \partial_{z^{\prime}} \psi_{n}\left(j, z^{\prime}\right)\right)\right|_{z^{\prime}=\epsilon},
$$

$\mathcal{C}(j, K, \epsilon)=\mathcal{H}(j, K, \epsilon)$

and the substitution $\psi_{n}(z \rightarrow 0) \approx c_{n}(j) z^{\Delta} L_{n}^{\Delta(j)-2}(0)$ for the soft-wall model. 
[1] E. A. Kuraev, L. N. Lipatov, and V. S. Fadin, Zh. Eksp. Teor. Fiz. 72, 377 (1977) [Sov. Phys. JETP 45, 199 (1977)].

[2] I. I. Balitsky and L. N. Lipatov, Yad. Fiz. 28, 1597 (1978) [Sov. J. Nucl. Phys. 28, 822 (1978)].

[3] J. M. Maldacena, Int. J. Theor. Phys. 38, 1113 (1999); Adv. Theor. Math. Phys. 2, 231 (1998); S. S. Gubser, I. R. Klebanov, and A. M. Polyakov, Phys. Lett. B 428, 105 (1998); E. Witten, Adv. Theor. Math. Phys. 2, 505 (1998); I. R. Klebanov and E. Witten, Nucl. Phys. B556, 89 (1999).

[4] M. S. Costa, M. Djuric, and N. Evans, J. High Energy Phys. 09 (2013) 084.

[5] C. H. Lee, H. Y. Ryu, and I. Zahed, Phys. Rev. D 98, 056006 (2018).

[6] M. Rho, S. J. Sin, and I. Zahed, Phys. Lett. B 466, 199 (1999).

[7] R. A. Janik and R. B. Peschanski, Nucl. Phys. B586, 163 (2000).

[8] R. C. Brower, J. Polchinski, M. J. Strassler, and C. I. Tan, J. High Energy Phys. 12 (2007) 005; R. C. Brower, M. J. Strassler, and C. I. Tan, J. High Energy Phys. 03 (2009) 092; R. C. Brower, M. S. Costa, M. Djurić, T. Raben, and C. I. Tan, J. High Energy Phys. 02 (2015) 104.

[9] A. Stoffers and I. Zahed, Phys. Rev. D 87, 075023 (2013); 88, 025038 (2013).

[10] A. Stoffers and I. Zahed, arXiv:1210.3724.

[11] G. Basar, D. E. Kharzeev, H. U. Yee, and I. Zahed, Phys. Rev. D 85, 105005 (2012).

[12] Y. Hatta, E. Iancu, and A. H. Mueller, J. High Energy Phys. 01 (2008) 026.

[13] Y. Hatta, T. Ueda, and B. W. Xiao, J. High Energy Phys. 08 (2009) 007.

[14] J. Nemchik, N. N. Nikolaev, and B. G. Zakharov, Phys. Lett. B 341, 228 (1994); J. Nemchik, N. N. Nikolaev, E. Predazzi, and B. G. Zakharov, Phys. Lett. B 374, 199 (1996); Z. Phys. C 75, 71 (1997); H. G. Dosch, T. Gousset, G. Kulzinger, and H. J. Pirner, Phys. Rev. D 55, 2602 (1997); G. Kulzinger, H. G. Dosch, and H. J. Pirner, Eur. Phys. J. C 7, 73 (1999); H. G. Dosch and E. Ferreira, Eur. Phys. J. C 51, 83 (2007); G. Chen, Y. Li, P. Maris, K. Tuchin, and J. P. Vary, Phys. Lett. B 769, 477 (2017); N. Nikolaev and B. G. Zakharov, Z. Phys. C 53, 331 (1992); A. H. Mueller and B. Patel, Nucl. Phys. B425, 471 (1994); J. R. Forshaw, G. Kerley, and G. Shaw, Phys. Rev. D 60, 074012 (1999); Nucl. Phys. A675, 80C (2000); M. McDermott, R. Sandapen, and G. Shaw, Eur. Phys. J. C 22, 655 (2002); K. J. Golec-Biernat and M. Wusthoff, Phys. Rev. D 59, 014017 (1998); 60, 114023 (1999); E. Iancu, K. Itakura, and S. Munier, Phys. Lett. B 590, 199 (2004); J. R. Forshaw, R. Sandapen, and G. Shaw, Phys. Rev. D 69, 094013 (2004); C. Marquet, R. B. Peschanski, and G. Soyez, Phys. Rev. D 76, 034011 (2007); J. R. Forshaw and R. Sandapen, J. High Energy Phys. 10 (2011) 093.

[15] A. Ali et al. (GlueX Collaboration), Phys. Rev. Lett. 123, 072001 (2019).

[16] K. Hafidi, S. Joosten, Z. E. Meziani, and J. W. Qiu, FewBody Syst. 58, 141 (2017); S. Joosten and Z. E. Meziani, Proc. Sci., QCDEV2017 (2018) 017 [arXiv:1802.02616].

[17] Y. Hatta and D. L. Yang, Phys. Rev. D 98, 074003 (2018).

[18] Y. Hatta, A. Rajan, and D. L. Yang, Phys. Rev. D 100, 014032 (2019).
[19] D. Kharzeev, H. Satz, A. Syamtomov, and G. Zinovjev, Eur. Phys. J. C 9, 459 (1999); S. J. Brodsky, E. Chudakov, P. Hoyer, and J. M. Laget, Phys. Lett. B 498, 23 (2001).

[20] M. Srednicki, Quantum Field Theory, edited by J. Wyley (Cambridge University Press, Cambridge, 2007).

[21] Z. Abidin and C.E. Carlson, Phys. Rev. D 79, 115003 (2009).

[22] E. D’Hoker and B. Pourhamzeh, J. High Energy Phys. 06 (2016) 146.

[23] I. Kirsch, J. High Energy Phys. 09 (2006) 052.

[24] H. R. Grigoryan, P. M. Hohler, and M. A. Stephanov, Phys. Rev. D 82, 026005 (2010).

[25] I. Kanitscheider, K. Skenderis, and M. Taylor, J. High Energy Phys. 09 (2008) 094.

[26] P. E. Shanahan and W. Detmold, Phys. Rev. D 99, 014511 (2019).

[27] M. V. Polyakov and P. Schweitzer, Int. J. Mod. Phys. A 33, 1830025 (2018).

[28] P. E. Shanahan and W. Detmold, Phys. Rev. Lett. 122, 072003 (2019).

[29] V. D. Burkert, L. Elouadrhiri, and F.X. Girod, Nature (London) 557, 396 (2018).

[30] U. Camerini, J. G. Learned, R. Prepost, C. M. Spencer, D. E. Wiser, W. W. Ash, R. L. Anderson, D. M. Ritson, D. J. Sherden, and C. K. Sinclair, Phys. Rev. Lett. 35, 483 (1975).

[31] B. Gittelman, K. M. Hanson, D. Larson, E. Loh, A. Silverman, and G. Theodosiou, Phys. Rev. Lett. 35, 1616 (1975).

[32] R. Barate et al. (NA14 Collaboration), Z. Phys. C 33, 505 (1987).

[33] M. E. Binkley et al., Phys. Rev. Lett. 48, 73 (1982).

[34] C. Adloff et al. (H1 Collaboration), Eur. Phys. J. C 13, 371 (2000).

[35] S. Chekanov et al. (ZEUS Collaboration), Eur. Phys. J. C 24, 345 (2002).

[36] A. Aktas et al. (H1 Collaboration), Eur. Phys. J. C 46, 585 (2006).

[37] C. Alexa et al. (H1 Collaboration), Eur. Phys. J. C 73, 2466 (2013).

[38] R. Aaij et al. (LHCb Collaboration), J. Phys. G 40, 045001 (2013).

[39] J. Breitweg et al. (ZEUS Collaboration), Phys. Lett. B 437, 432 (1998).

[40] C. Adloff et al. (H1 Collaboration), Phys. Lett. B 483, 23 (2000).

[41] S. Chekanov et al. (ZEUS Collaboration), Phys. Lett. B 680, 4 (2009).

[42] R. Aaij et al. (LHCb Collaboration), J. High Energy Phys. 09 (2015) 084.

[43] A. M. Sirunyan et al. (CMS Collaboration), Eur. Phys. J. C 79, 277 (2019).

[44] A. V. Belitsky and A. V. Radyushkin, Phys. Rep. 418, 1 (2005).

[45] A. Vega, I. Schmidt, T. Gutsche, and V. E. Lyubovitskij, Phys. Rev. D 83, 036001 (2011).

[46] X. D. Ji, Phys. Rev. Lett. 78, 610 (1997).

[47] H. R. Grigoryan and A. V. Radyushkin, Phys. Lett. B 650 , 421 (2007).

[48] Z. Abidin and C.E. Carlson, Phys. Rev. D 77, 095007 (2008). 
[49] H. R. Grigoryan and A. V. Radyushkin, Phys. Rev. D 76, 095007 (2007).

[50] S. Hong, S. Yoon, and M. J. Strassler, J. High Energy Phys. 04 (2006) 003.

[51] C. A. B. Bayona, H. Boschi-Filho, and N. R. F. Braga, J. High Energy Phys. 03 (2008) 064.

[52] J. H. Gao and B. W. Xiao, Phys. Rev. D 81, 035008 (2010).
[53] S. Raju, Phys. Rev. D 83, 126002 (2011).

[54] E. D'Hoker, D. Z. Freedman, S. D. Mathur, A. Matusis, and L. Rastelli, Nucl. Phys. B562, 330 (1999).

[55] J. Polchinski and M. J. Strassler, Phys. Rev. Lett. 88, 031601 (2002); R. C. Brower, M. J. Strassler, and C. I. Tan, J. High Energy Phys. 03 (2009) 092. 